\title{
Distinguished quantum states in a class of cosmologi- cal spacetimes and their Hadamard property
}

\author{
Claudio Dappiaggi ${ }^{1, a}$, Valter Moretti ${ }^{2, b}$, Nicola Pinamonti ${ }^{1, c}$ \\ ${ }^{1}$ II. Institut für Theoretische Physik, Universität Hamburg, Luruper Chaussee 149, D-22761 Hamburg, Germany. \\ ${ }^{2}$ Dipartimento di Matematica, Università di Trento and Istituto Nazionale di Alta Matematica - Unità locale di \\ Trento - and Istituto Nazionale di Fisica Nucleare - Gruppo Collegato di Trento, \\ via Sommarive 14 I-38050 Povo (TN), Italy. \\ E-mail: ${ }^{a}$ claudio.dappiaggi@desy.de, ${ }^{b}$ moretti@science.unitn.it, ${ }^{c}$ nicola.pinamonti@desy.de
}

\begin{abstract}
In a recent paper, we proved that a large class of spacetimes, not necessarily homogeneous or isotropous and relevant at a cosmological level, possesses a preferred codimension one submanifold, i.e., the past cosmological horizon, on which it is possible to encode the information of a scalar field theory living in the bulk. Such bulk-toboundary reconstruction procedure entails the identification of a preferred quasifree algebraic state for the bulk theory, enjoying remarkable properties concerning invariance under isometries (if any) of the bulk and energy positivity, and reducing to well-known vacua in standard situations. In this paper, specialising to open FRW models, we extend previously obtained results and we prove that the preferred state is of Hadamard form, hence the backreaction on the metric is finite and the state can be used as a starting point for renormalisation procedures. That state could play a distinguished role in the discussion of the evolution of scalar fluctuations of the metric, an analysis often performed in the development of any model describing the dynamic of an early Universe which undergoes an inflationary phase of rapid expansion in the past.
\end{abstract}

Pacs: 04.62.+v, 98.80.Jk

\section{Introduction}

If one had to carry out a survey in the community of physicists asking for the field of expertise, from which we can expect in the next few years new exciting and unexpected developments, cosmology would be, if not an unanimous, certainly one of the most frequent answers.

According to the most commonly accepted idea, it is conceivable that, at large scales, a good model of the geometry of our Universe is given by an homogeneous and isotropic background whose metric is of Friedmann-Robertson-Walker (FRW) type and whose dynamic is ruled by the Einstein's equation supplemented with a suitable choice of the ordinary matter. This particular scenario is usually called cold dark matter model. Alas, such an approach is not devoid of some flaws and, according to modern theoretical cosmology, these can be solved or circumvented assuming that the Universe undergoes an early phase of rapid expansion, known as the inflation; moreover such model has the added-on advantage of entailing several direct and somehow simple explanations for observed phenomena such as the anisotropies of the spectrum of the cosmic microwave background, to quote one, if the most most notable example. A further remarkable property of many but not all inflationary models is related to the underlying geometry 
of the background which, at the time of the rapid expansion, turns out to be describable by a de-Sitter spacetime, where a very large effective cosmological constant appears.

Although the underlying assumptions of all FRW models, namely homogeneity and isotropy, are overidealisations, it is nonetheless safe to claim that a further distinguished advantage of this line of reasoning lies in the possibility to explicitly account for inhomogeneities as well as for anisotropies in terms of suitably modelled perturbations of the metric, they being either of scalar, vector or of tensor form (there is a vast literature and an interested reader should start looking at [BST83, MFB92] as well as at [HW02] for a careful discussion of the relations between these mentioned fluctuations and the inflationary models). Both from a mathematical and from a physical perspective, it is rather interesting to notice that these perturbations are not supposedly classical but they are truly of quantum nature. Without entering into details, they are thought as originating during the phase of rapid expansion as fluctuations over a suitable ground state and, later, they are let freely evolve. To corroborate such assumption, it has been shown in the past years how it is possible to retrieve by means of these techniques an (almost) scale-free power spectrum, also known as the Harrison-Zeldovich power spectrum.

Although rather compelling, the above picture cannot be seen as mathematically sound since all the performed analyses rely on the existence and on the notion of a suitable ground state, a concept which unfortunately is highly non trivial to set-up for quantum field theories on curved backgrounds. The aim of this paper will be, indeed, to make this idea precise though we shall only consider free scalar fields thought as a prototype for the metric perturbations. Particularly, we will construct and analyse a preferred state in a class of FRW spacetimes which are of de Sitter form as the cosmological time $\tau \rightarrow-\infty$ in a way we shall better specify in the main body of the paper. Most notably we shall show that such a state satisfies the Hadamard property [Wa94, KW91]. Roughly speaking and in a physical language it entails both that the ultraviolet divergences, present at the level of the two-point correlation functions between the fields, are similar in form to those arising if we deal with the Minkowski vacuum, and that the variance of the expectation values of any observable obtained as a limit of coincidence of arguments (i.e., Wick polynomials of the fields and their derivatives) cannot be divergent [HW01]. Therefore such state plays the role of a natural starting point in the investigation of interacting field theories by means of perturbative techniques. Particularly the stress-energy tensor operator [Mo03], averaged with respect to the state, is finite as well as the backreaction on the metric; therefore the model may be gravitationally stable at least at perturbative level (see [DFP08] for an example in a cosmological set up).

The main peculiarity of this paper is that the construction of our proposed state originates from a different albeit related scenario which traces its origins back to G. 't Hooft formulation of the holographic principle - see [Ho93] - which advocates the existence of a strong interplay between field theories constructed on manifolds of different dimensions. Though often associated either to AdS [Reh00, DR03, Ri07] or to asymptotically flat spacetimes [DMP06, Mo06, Da07], in [DMP08], we showed that a bulk-toboundary reconstruction procedure can be successfully set up also in a large class of expanding spacetimes, not necessarily homogeneous or isotropous, relevant at a cosmological level. Assuming homogeneity and isotropy, these encompass, as a subclass, the Friedmann-Robertson-Walker spacetimes with flat spatial section which satisfy a suitable constraint on the expansion factor $a(t)$, namely, as the conformal time $\tau \rightarrow-\infty$, the leading behaviour of $a(\tau)$ is that of the cosmological de Sitter spacetime.

Furthermore, it turns out that all the manifolds satisfying the hypotheses formulated in [DMP08] posses a preferred codimension one submanifold, namely, the so-called (past) cosmological horizon $\Im^{-}$, on which we can encode the information of a scalar field theory on the bulk, barring some further constraints both on the mass and on the coupling to scalar curvature. As in the asymptotically flat scenario [DMP06, Mo06, Mo08], we also managed to identify preferred quasifree algebraic state $\lambda$ associated to such boundary theory; $\lambda$ satisfies a uniqueness property and, furthermore, it is invariant under a suitable notion of asymptotic-symmetry group introduced in [DMP08] for general (not homogeneous or isotropous) 
expanding universes with geodetically-complete past cosmological horizon. $\lambda$ is universal, in the sense that it does not depend on the particular bulk spacetime $M$ admitting $\Im^{-}$as past boundary. However, fixing one of those spacetimes $M, \lambda$ can be pulled-back in the bulk $M$ to identify a new quasifree state $\lambda_{M}$ which is a natural candidate to play the role of a preferred state associated to the bulk theory, since it fulfils several relevant properties: (1) it is invariant under every isometry (if any) of the bulk which preserves the structure of $\Im^{-},(2) \lambda_{M}$ admits positive energy with respect to every timelike Killing isometry of $M$ (if any) which preserves the structure of $\Im^{-}$, (3) $\lambda_{M}$ reduces to the well-known Bunch-Davies vacuum when $M$ is the very de Sitter spacetime. As already stated, our main goal here is to prove that it is also of Hadamard form.

It is also remarkable to notice that these results could be easily extended to other inflationary scenarios as for example that of the "power law" model. To wit the dynamical content of the underlying quantum fields when considered on the dual Minkowski metric (see equation (2)) mimics the one taken here into account. In those cases the potential in (9) which governs the time evolution of the fields with respect to the Minkowski time $\tau$ is still of the form $C \tau^{-2}+O\left(\tau^{-3}\right)$. In other words those theories are conformally related to the case investigated in the present paper, hence, since the Hadamard property is invariant under conformal transformations, the definition of a preferred state and the study of its ultraviolet divergences could be dealt with in an analogous way.

It is important to notice that there have been previous results tackling the problem to construct Hadamard states on FRW spacetimes. It is worth mentioning Olberman's result [O107], which is based on a previous analysis due to Lüders and Roberts [LR90] and to Junker and Schrohe [JS01]. The difference of our approach is in the choice of a different approximation prescription used in the explicit construction of the modes, in the present paper instead of considering the adiabatic approximation we shall explicitly discuss both the construction of modes out of their asymptotic behaviour and the issue of the convergence of the arising perturbative series by means of the so called Green function method. A further recent result, dwelling in the construction of Hadamard states with nice thermal properties is presented in the PhD thesis of Küskü [Kü08].

The outline of the paper is the following: in the next section we briefly recollect some of the results of [DMP08] and, particularly, we discuss the main geometric properties of the backgrounds we shall consider identifying a preferred codimension 1 submanifold, namely, the cosmological horizon. In section 2 we set up the bulk-to-boundary reconstruction procedure for a scalar field and we identify a preferred algebraic quasifree bulk state, extending some results previously achieved in [DMP08]. Furthermore, we shall discuss the regularity properties of the solutions of the bulk equations of motion once they are restricted on the horizon and once suitable constraints on the mass $m$ and on the coupling to scalar curvature $\xi$ are imposed. In the third section we prove the main result of the paper, i.e., the two-point function associated to the constructed state in the bulk is a well-defined distribution of Hadamard form. Eventually in the fourth section, we briefly draw some conclusions.

1.1. Expanding Friedmann-Robertson-Walker spacetime with flat spatial sections. Let us remind the reader some geometric properties of the spacetimes we intend to consider. As this topic has been dealt with in greater details in [DMP08], in this section we shall present only the ingredients relevant to understand the main statements of this paper, while pointing a reader interested in further discussions and details to [DMP08].

The homogeneous and isotropic solutions of Einstein's equation which are of cosmological interest can be described as a four dimensional smooth Lorentzian manifold $M$ equipped with the following metric:

$$
g=-d t^{2}+a^{2}(t)\left[\frac{d r^{2}}{1-k r^{2}}+r^{2} d \mathbb{S}^{2}(\theta, \varphi)\right]
$$


Here $k$ takes the values $-1,0,1$ and it indicates whether the constant time hypersurfaces are respectively hyperbolic, flat or parabolic, whereas $a(t)$ is a smooth function of constant sign depending only on the variable $t$, whose domain ranges in an open interval $I=(\alpha, \beta)$. Such class of backgrounds is too large for our purposes and, therefore, we shall henceforth restrict our attention to the subclass with both $k=0$ and $\dot{a}(t) \geq 0$. It represents, at a physical level, a remarkably interesting case of a globally-hyperbolic Friedmann-Robertson-Walker (FRW) expanding Universe $\left(M, g_{F R W}\right)$ with flat spatial sections. $M$ is diffeomorphic to $I \times \mathbb{R}^{3}$ and, up to a change of coordinates, the metric reads

$$
g_{F R W} \doteq a^{2}(\tau)\left[-d \tau^{2}+d r^{2}+r^{2} d \mathbb{S}^{2}(\theta, \varphi)\right] .
$$

Here $\tau$ is the so-called conformal time constructed out of the defining identity $a(\tau) d \tau \doteq d t$. As a last restriction, we require the conformal factor to have the following form referring to its behaviour as $\tau \rightarrow-\infty:$

$$
a(\tau)=-\frac{1}{H \tau}+O\left(\frac{1}{\tau^{2}}\right), \quad \frac{d a(\tau)}{d \tau}=\frac{1}{H \tau^{2}}+O\left(\frac{1}{\tau^{3}}\right), \quad \frac{d^{2} a(\tau)}{d \tau^{2}}=-\frac{1}{H \tau^{3}}+O\left(\frac{1}{\tau^{4}}\right) .
$$

The reason underlying such constraints is twofold. On the one hand they identify a class of physically relevant spacetimes with a rather distinguished geometric property, namely they all posses a preferred codimension one submanifold which represents the natural screen on which to encode the data of a bulk field theory. On the other hand (2) and (3) characterise those backgrounds which look "asymptotically" - i.e., as $\tau \rightarrow-\infty$ - as the cosmological de Sitter Universe, whose expansion factor in (2) is

$$
a_{d S}(\tau) \doteq-\frac{1}{H \tau}
$$

and $\tau \in I=(-\infty, \beta)$. This property is shared also by all others solutions of (2) satisfying (3). Above $H$ is the so-called Hubble parameter and we felt safe to adopt the same symbol also in the preceding formulas.

The class of spacetimes we characterised has a quite remarkable application in the description of the early stages of the evolution of the universe, since most of the so-called inflationary scenarios are based on a phase of rapid expansion modelled by a scalar field on an (asymptotically) de Sitter background.

1.2. Past cosmological horizons. As we discussed in detail in [DMP08], all the spacetimes under analysis are globally hyperbolic - the constant- $\tau$ hypersurfaces being trivially smooth spacelike Cauchy surfaces - and they posses a boundary made of a null codimension one-submanifold $\Im^{-}$on which to encode the data of a bulk field-theory. This is the so-called (past) cosmological horizon as defined by Rindler in [Ri06]. To concretely characterise $\Im^{-}$, first one performs the coordinate change $U=\tan ^{-1}(\tau-r)$, $V=\tan ^{-1}(\tau+r)$; in this way one can realize by direct inspection that $\left(M, g_{F R W}\right)$ could be read as an open submanifold of a larger spacetime $(\widehat{M}, \widehat{g})$, i.e., $M \subset \widehat{M}$ and $\left.\widehat{g}\right|_{M} \equiv g_{F R W}$. In this framework, $\Im^{-}$is nothing but the past causal boundary of $M$ in $\widehat{M}$, that is $M=I^{+}\left(\Im^{-} ; \widehat{M}\right)$ and $\Im^{-}=\partial M=\partial J^{+}(M, \widehat{M})$. $\Im^{-}$turns out to be diffeomorphic to $\mathbb{R} \times \mathbb{S}^{2}$ as well as a null differentiable manifold. We shall also exploit some further remarkable geometric properties valid in a neighbourhood of $\Im^{-}$, namely $a(\tau(U, V))$ vanishes identically on $\Im^{-}$whereas the differential $\left.d a(U, V)\right|_{\Im^{-}}=-2 H^{-1} d V$ does not. Such feature entails that the metric $\widehat{g}$ can be restricted on the horizon where it picks up a (geodetically complete) Bondi-like form:

$$
\left.\widehat{g}\right|_{\Im^{-}}=H^{-2}\left(-2 d \ell d a+d \mathbb{S}^{2}(\theta, \varphi)\right) .
$$

Here $d \mathbb{S}^{2}$ is the standard metric on the 2 -sphere, whereas, up to a constant, $\ell(V)=-H \tan (V)$, is the affine parameter of the integral line of the vector $n \doteq \nabla a$ which turns out to be a complete null geodesics 
of $\widehat{g}$. Furthermore the above remark on the form of the expansion factor and of its derivative on the horizon also leads to conclude $\mathcal{L}_{\partial_{\tau}} \widehat{g}=-2 \partial_{\tau}(\ln a) \widehat{g}$, where the right hand side vanishes on $\Im^{-}$as $a$ does. Hence we can infer that $\partial_{\tau}$ is a conformal Killing vector which, approaching $\Im^{-}$, both tends to become tangent to it and to coincide with $-H^{-1} \widehat{\nabla}^{b} a$. Such property will come in very handy in the forthcoming discussions.

Remark 1.1. In this paper we shall confine ourselves to FRW models. However, we stress that, as discussed in [DMP08], most results presented therein are valid also for spacetimes which are neither homogeneous nor isotropous, when they admit a geodetically-complete past cosmological horizon and a preferred conformal time $\partial_{\tau}$ with the previously discussed interplay with the geometry of the horizon. From a very abstract point of view, as established in [DMP08], $\Im^{-}$can be equipped with a certain infinitedimensional group of isometries $S G_{\Im^{-}}$which is the analogue of the BMS group for asymptotically flat spacetimes [DMP06]. This group, on the one hand depends on the only structure of $\Im^{-}$, hence in this sense is universal, while, on the other hand, it embodies - through a faithful representation - all possible bulk Killing symmetries which preserve the structure of $\Im^{-}$of every - not necessarily homogeneous or isotropous - cosmological model which admits $\Im^{-}$as a boundary (see proposition 3.2 as discussed in [DMP08]).

Also in the general case considered in [DMP08], $\Im^{-}$is a natural candidate on which to encode the information of bulk data of the scalar free QFT. As we shall shortly discuss in the particular case considered in this paper, it has been proved in [DMP08] that it is possible both to construct a genuine free scalar quantum field theory defined on $\Im^{-}$and to associate to it a preferred state enjoying invariance under $S G_{\Im^{-}}$. Such theory can be induced back to the bulk giving rise to a second one which turns out to be automatically invariant under every symmetry of the bulk which preserves $\Im^{-}$.

\section{From the bulk to the boundary and back}

The aim of this subsection is to sketch the scheme of quantisation of a free scalar field living on any of the spacetimes under analysis, as previously discussed in [DMP08], focusing on how it is possible to associate every quantum theory living in $\left(M, g_{F R W}\right)$ with a dual theory on $\Im^{-}$. The main advantage of pursuing such approach lies in the existence of a preferred quasifree state (see remark 1.1) for the boundary theory that we shall eventually pullback to the bulk, picking out a natural preferred quasifree state $\lambda_{M}$ for the QFT in the spacetime $\left(M, g_{R F W}\right)$. The state $\lambda_{M}$ generalises the Bunch-Davies vacuum for more general expanding universes. The analysis of the remarkable properties of $\omega_{M}$ was started in [DMP08] for a wider class of spacetimes (dropping the requirements of homogeneity and isotropy); here we only focus on the validity of the Hadamard property for $\lambda_{M}$ in the class of RFW spacetimes we are considering.

Since $\left(M, g_{F R W}\right)$ is globally hyperbolic, the Cauchy problem for smooth compactly-supported initial data is well-posed [Wa94, BGP96]. Let us thus consider the Klein-Gordon equation for the real scalar field $\Phi$ arbitrarily coupled $\xi$ with the scalar curvature:

$$
P \Phi=0, \quad \text { where } P=-\square+\xi R+m^{2},
$$

where $\square$ is the D'Alembert operator associated with $g_{F R W}$ and $m^{2} \geq 0$. The space of real smooth solutions $\mathcal{S}(M)$ of $(5)$ with compactly supported smooth Cauchy data is a symplectic space $\left(\mathcal{S}(M), \sigma_{M}\right)$ when endowed with the Cauchy-surface independent nondegenerate symplectic form:

$$
\sigma_{M}\left(\Phi_{1}, \Phi_{2}\right) \doteq \int_{\Sigma} d \mu_{g_{F R W}}^{(\Sigma)}\left(\Phi_{2} \nabla_{n} \Phi_{1}-\Phi_{1} \nabla_{n} \Phi_{2}\right), \quad \forall \Phi_{1}, \Phi_{2} \in \mathcal{S}(M) .
$$


Above, $\Sigma$ is an arbitrary spacelike smooth Cauchy surface, $d \mu_{g_{F R W}}^{(\Sigma)}$ the measure on $\Sigma$ induced by the metric and $n$ is the unit future-pointing vector orthogonal to $\Sigma$. It is then a standard procedure [KW91, Wa94, BR022] to associate $\left(\mathcal{S}(M), \sigma_{M}\right)$ with the Weyl $C^{*}$-algebra $\mathcal{W}(M)$, determined up to isometric *-isomorphisms and constructed out of the Weyl generators $W_{M}(\Phi) \neq 0$, satisfying, for all $\Phi \in \mathcal{S}(M)$, the Weyl relations

$$
W_{M}(\Phi)=W_{M}^{*}(-\Phi), \quad W_{M}(\Phi) W_{M}\left(\Phi^{\prime}\right)=e^{\frac{i}{2} \sigma_{M}\left(\Phi, \Phi^{\prime}\right)} W_{M}\left(\Phi+\Phi^{\prime}\right) .
$$

The self-adjoint elements of $\mathcal{W}(M)$ represent the quantum observable of the bosonic free quantum field theory of the field $\Phi$ and hence $\mathcal{W}(M)$ realizes the quantisation of the theory at algebraic level [Wa94, KW91, BR021, BR022].

2.1. Modes. We wish now to better characterise $\mathcal{S}(M)$ employing constant-time hypersurfaces $\Sigma_{\tau}$ (which are diffeomorphic to $\mathbb{R}^{3}$ ) as Cauchy surfaces and adopting standard Cartesian coordinates $(\tau, \vec{x})$ on $M$ as in (2). Adopting the convention that $\mathbf{k} \in \mathbb{R}^{3}$ and $k=|\mathbf{k}|$, a generic element $\Phi \in \mathcal{S}(M)$ can be decomposed as:

$$
\Phi(\tau, \vec{x})=\int_{\mathbb{R}^{3}} d^{3} \mathbf{k}\left[\phi_{\mathbf{k}}(\tau, \vec{x}) \widetilde{\Phi}(\mathbf{k})+\overline{\phi_{\mathbf{k}}(\tau, \vec{x}) \widetilde{\Phi}(\mathbf{k})}\right] \quad \text { with } \quad \phi_{k}(\tau, \vec{x}) \doteq \frac{e^{i \mathbf{k} \cdot \vec{x}}}{(2 \pi)^{\frac{3}{2}}} \frac{\chi_{k}(\tau)}{a(\tau)}
$$

The modes $\phi_{\mathbf{k}}$ are constructed out of the $\chi_{k}(\tau)$, which are solutions of the differential equation:

$$
\begin{gathered}
\frac{d^{2}}{d \tau^{2}} \chi_{k}(\tau)+\left(V_{0}(k, \tau)+V(\tau)\right) \chi_{k}(\tau)=0, \\
V(\tau) \doteq k^{2}+a(\tau)^{2}\left[m^{2}+\left(\xi-\frac{1}{6}\right) R(\tau)\right]-V_{0}(k, \tau),
\end{gathered}
$$

where $V_{0}(k, \tau) \doteq k^{2}+a_{d S}(\tau)^{2}\left[m^{2}+\left(\xi-\frac{1}{6}\right) 12 H^{2}\right]$, so that it results $V(\tau)=O\left(1 / \tau^{3}\right)$ in view of $(3)$ as $\tau \rightarrow-\infty$. The following condition is also assumed:

$$
\frac{\overline{d \chi_{k}(\tau)}}{d \tau} \chi_{k}(\tau)-\overline{\chi_{k}(\tau)} \frac{d \chi_{k}(\tau)}{d \tau}=i, \quad \tau \in \mathbb{R}_{-} .
$$

We now define:

$$
\nu=\sqrt{\frac{9}{4}-\left(\frac{m^{2}}{H^{2}}+12 \xi\right)}, \quad \text { where we always assume both } \operatorname{Re} \nu \geq 0 \text { and } \operatorname{Im} \nu \geq 0
$$

noticing that $\nu$ can be either real or imaginary, but not a general complex number. A general solution of (9) satisfying the constraint (10) and with $R e \nu<1 / 2$ can be constructed as a convergent series, as discussed in Theorem 4.5 of [DMP08]. Therein, $V(\tau)$ is treated as a perturbation potential over the solutions (12) in de Sitter background where $V \equiv 0$. For the purely de Sitter spacetime $(V \equiv 0)$ solutions of (9) satisfying the constraint (10) are fixed to be:

$$
\chi_{k}(\tau)=\frac{\sqrt{-\pi \tau}}{2} e^{\frac{-i \pi \nu}{2}} \overline{H^{(2)}} \nu(-k \tau),
$$

where $H_{\nu}^{(2)}$ is the Hankel function of second kind [SS76]. Moreover the perturbative procedure to construct the modes $\chi_{k}(\tau)$ for the general background yields the constraints (valid for the pure de Sitter case, too):

$$
\lim _{\tau \rightarrow-\infty} \chi_{k}(\tau) e^{i k \tau}=\frac{e^{-i \pi / 4}}{\sqrt{2 k}}, \quad \lim _{\tau \rightarrow-\infty} \frac{d \chi_{k}}{d \tau}(\tau) e^{i k \tau}=-i e^{-i \pi / 4} \sqrt{\frac{k}{2}} .
$$




\section{Remark 2.1.}

(1) The perturbative construction of the smooth real solutions of the Klein-Gordon equation with compactly supported data, as presented in Theorem 4.5 in [DMP08], extends with minor changes to the case $\operatorname{Re} \nu<3 / 2$ provided that the potential decays as $V(\tau)=O\left(\frac{1}{\tau^{5}}\right)$, which corresponds to the stricter constraints on the rate of expansion $a(\tau)$ :

$$
a(\tau)=-\frac{1}{H \tau}+O\left(\frac{1}{\tau^{5 / 2}}\right), \quad \frac{d a(\tau)}{d \tau}=\frac{1}{H \tau^{2}}+O\left(\frac{1}{\tau^{3}}\right), \quad \frac{d^{2} a(\tau)}{d \tau^{2}}=-\frac{1}{H \tau^{3}}+O\left(\frac{1}{\tau^{6}}\right) .
$$

Nonetheless we feel that, despite such further restriction on the choice of the underlying geometry, it is interesting from a physical perspective to allow $\nu$ to be as close to $\frac{3}{2}$ as possible. As a matter of facts, in this case the power spectrum $P(k, \tau)$ of the scalar field will be close to the Harrison-Zeldovich scale free one. As an evidence of this claim, notice that, at least at small scales and as $\tau \rightarrow-\infty$,

$$
P(k, \tau)=\overline{\chi_{k}(\tau)} \chi_{k}(\tau) \sim \frac{1}{|k|^{2 \nu}}
$$

as one can infer from the analysis performed in the appendix A. If we set $\nu=3 / 2$, then we end up with a genuine scale free spectrum.

(2) The identity (8) inverts as:

$$
\widetilde{\Phi}(\mathbf{k})=-i \int_{\Sigma_{\tau}} d^{3} x a^{2}(\tau)\left[\frac{\partial \overline{\phi_{\mathbf{k}}(\tau, \vec{x})}}{\partial \tau} \Phi(\tau, \vec{x})-\overline{\phi_{\mathbf{k}}(\tau, \vec{x})} \frac{\partial \Phi(\tau, \vec{x})}{\partial \tau}\right],
$$

$\Sigma_{\tau}$ being any constant-time hypersurface. As the right hand side is independent from the choice of a specific value $\tau$, we are free to let $\Sigma_{\tau}$ coincide with the Cauchy surface. In this way, $\Phi(\tau, \vec{x})$ and $\frac{\partial \Phi}{\partial \tau}(\tau, \vec{x})$ are the assigned initial data of the considered element $\Phi \in \mathcal{S}(M)$ individuated by $\widetilde{\Phi}$ inserted in the right-hand side of (8).

(3) Out of the behaviour of Hankel functions in a neighbourhood of the origin and in the perturbative construction of the general solution valid both for $\operatorname{Re\nu }<1 / 2$ and $V(\tau)=O\left(\frac{1}{\tau^{3}}\right)$, or for $\operatorname{Re\nu }<3 / 2$ and $V(\tau)=O\left(\frac{1}{\tau^{5}}\right)$ (see in the appendix A for more details), the shape of $\widetilde{\Phi}$ as $\mathbf{k} \rightarrow 0$ is as follows: whether $\nu$ is imaginary, no singularity occurs, whereas, if $R e \nu>0$, following the analysis performed in the proof of Theorem 4.5 in [DMP08] (and its extension to the case $R e \nu<3 / 2$, in appendix (A)), one gets that

$$
\left|\partial_{k}^{n} \widetilde{\Phi}(\mathbf{k})\right| \leq C_{\delta, n} /|\mathbf{k}|^{R e \nu+n}, \quad \text { for } 0<|\mathbf{k}| \leq \delta,
$$

if $n=0,1,2,3$ and for some $C_{\delta, n}, \delta>0$. These estimates arise for the analogous of the functions $k \mapsto \chi_{k}(\tau), k \mapsto \partial_{\tau} \chi_{k}(\tau)$ and their $k$-derivatives.

For $|\mathbf{k}| \rightarrow+\infty$ we have instead the following behaviour. As the $k \mapsto \chi_{k}(\tau), k \mapsto \partial_{\tau} \chi_{k}(\tau)$ and their $k$ derivatives increase at most polynomially and since the Cauchy data are smooth and compactly supported, (15) entails that $\widetilde{\Phi} \in C^{\infty}\left(\mathbb{R}^{3} \backslash\{0\} ; \mathbb{C}\right)$ and, for every $\Delta>0, n, m=0,1,2, \ldots$ there are constants $B_{\Delta, n, m}$ with:

$$
\left|\partial_{k}^{n} \widetilde{\Phi}(\mathbf{k})\right| \leq B_{\Delta, n, m} /|\mathbf{k}|^{m}, \quad \text { for }|\mathbf{k}| \geq \Delta .
$$

2.2. Projection of the quantum theory on the Horizon. Let us now focus our attention on the horizon itself. Since $\Im^{-}$is diffeomorphic to $\mathbb{R} \times \mathbb{S}^{2}$, we adopt the coordinates $(\ell, \theta, \varphi) \in \mathbb{R} \times \mathbb{S}^{2}$ used in (4). 
We want to define a suitable symplectic space in order to construct the Weyl algebra of the observables defined on a null surface as $\Im^{-}$[MP03, DMP06, DMP08]. To this end, the introduction of some useful mathematical tools is in due course.

The complex smooth functions which decay, with every derivative, faster than every negative power of $\ell$ uniformly in the angular variables) will be indicated by $\mathscr{S}\left(\mathbb{R} \times \mathbb{S}^{2}\right)$. Notice that, if $f \in \mathscr{S}\left(\mathbb{R} \times \mathbb{S}^{2}\right)$, then $f(\cdot, \omega) \in \mathscr{S}(\mathbb{R})$ for every fixed $\omega \in \mathbb{S}^{2}$. The complete dual space (with respect to the natural Frechét topology) of $\mathscr{S}\left(\mathbb{R} \times \mathbb{S}^{2}\right)$ will be denoted by $\mathscr{S}^{\prime}\left(\mathbb{R} \times \mathbb{S}^{2}\right)$. In the following $\widehat{\psi}$ denotes the Fourier transform ${ }^{1}$ of the distribution $\psi \in \mathscr{S}^{\prime}\left(\mathbb{R} \times \mathbb{S}^{2}\right)$. As in the standard theory, this transformation is defined by assuming that, if $\phi \in \mathscr{S}\left(\mathbb{R} \times \mathbb{S}^{2}\right)$,

$$
\widehat{\phi}(k, \omega) \doteq \int_{\mathbb{R}} \frac{e^{i k \ell}}{\sqrt{2 \pi}} \psi(\ell, \omega) d \ell, \quad \forall(k, \omega) \in \mathbb{R} \times \mathbb{S}^{2},
$$

so that $\widehat{\phi} \in \mathscr{S}\left(\mathbb{R} \times \mathbb{S}^{2}\right)$ again, and afterwards, extending the definition per duality to $T \in \mathscr{S}^{\prime}\left(\mathbb{R} \times \mathbb{S}^{2}\right)$ as $\langle\widehat{T}, \phi\rangle \doteq\langle T, \widehat{\phi}\rangle$ for all $\phi \in \mathscr{S}\left(\mathbb{R} \times \mathbb{S}^{2}\right)$. The Fourier transform turns out to be bijective and continuous both as a map $\mathscr{S}\left(\mathbb{R} \times \mathbb{S}^{2}\right) \rightarrow \mathscr{S}\left(\mathbb{R} \times \mathbb{S}^{2}\right)$ and $\mathscr{S}^{\prime}\left(\mathbb{R} \times \mathbb{S}^{2}\right) \rightarrow \mathscr{S}^{\prime}\left(\mathbb{R} \times \mathbb{S}^{2}\right)$ whereas the inverse transform is obtained by duality starting from the inverse Fourier transform on $\mathscr{S}\left(\mathbb{R} \times \mathbb{S}^{2}\right)$ :

$$
\psi(\ell, \omega) \doteq \int_{\mathbb{R}} \frac{e^{-i k \ell}}{\sqrt{2 \pi}} \widehat{\phi}(k, \omega) d \ell, \quad \forall(\ell, \omega) \in \mathbb{R} \times \mathbb{S}^{2},
$$

If $\phi \in L^{1}\left(\mathbb{R} \times \mathbb{S}^{2}, d \ell d S^{2}\right)$, so that $\phi \in \mathscr{S}^{\prime}\left(\mathbb{R} \times \mathbb{S}^{2}\right)$, its Fourier transform, can be equivalently computed as the right-hand side of (18) and $\widehat{\phi}$ is $k$-continuous.

Using these tools, as a first step to define a bosonic field theory, we introduce the symplectic space of real wavefunctions $\left(\mathcal{S}\left(\Im^{-}\right), \sigma\right)$ relaxing the requirements on the elements of the space with respect to that done in [DMP08] in order to encompass the physically interesting case $R e \nu<3 / 2$ as we shall see shortly:

$$
\begin{gathered}
\mathcal{S}\left(\Im^{-}\right) \doteq\left\{\psi \in C^{\infty}\left(\mathbb{R} \times \mathbb{S}^{2}\right) \mid\|\psi\|_{\infty},\|k \widehat{\psi}\|_{\infty}<\infty, \partial_{\ell} \psi \in L^{1}\left(\mathbb{R} \times \mathbb{S}^{2}, d \ell d S^{2}\right), \widehat{\psi} \in L^{1}\left(\mathbb{R} \times \mathbb{S}^{2}, d k d S^{2}\right)\right\}, \\
\sigma_{\Im^{-}}\left(\psi, \psi^{\prime}\right) \doteq \int_{\mathbb{R} \times \mathbb{S}^{2}}\left(\psi \frac{\partial \psi^{\prime}}{\partial \ell}-\psi^{\prime} \frac{\partial \psi}{\partial \ell}\right) d \ell d S^{2}, \quad \forall \psi, \psi^{\prime} \in S\left(\Im^{-}\right) .
\end{gathered}
$$

Notice that above, where $\psi \in C^{\infty}\left(\mathbb{R} \times \mathbb{S}^{2}\right)$ and it is bounded, the Fourier transform $\widehat{\psi}$ makes sense in the distributional sense. In [DMP08], $\mathcal{S}\left(\Im^{-}\right)$was defined as the space of smooth real-valued functions of $L^{2}\left(\mathbb{R} \times \mathbb{S}^{2} ; d \ell d S^{2}\right)$ with $\ell$-derivative in $L^{2}\left(\mathbb{R} \mathbb{S}^{2} ; d \ell d S^{2}\right)$. In that case the Fourier transform could be interpreted as a Fourier-Plancherel transform. In our case this is not possible in general.

Since $\sigma_{\Im^{-}}$is non-degenerate, it is possible to associate to $\left(S\left(\Im^{-}\right), \sigma_{\Im^{-}}\right)$a unique, up to isometric $*-$ isomorphism, Weyl $C^{*}$-algebra $\mathcal{W}\left(\Im^{-}\right)$whose generators $W_{\Im^{-}}(\psi) \neq 0$ for $\psi \in S\left(\Im^{-}\right)$satisfy the Weyl relations (7) with $W_{M}$ replaced by $W_{\Im^{-}}, \sigma_{M}$ replaced by $\sigma$ and $\Phi, \Phi^{\prime}$ replaced by $\psi, \psi^{\prime}$. As for the bulk, $\mathcal{W}\left(\Im^{-}\right)$represents a well-defined set of basic observables and, hence, it can be thought as the building block of a full-fledged quantum scalar field theory on the cosmological horizon $\Im^{-}$.

Nonetheless such line of reasoning would be spurious if we were not able to connect the information

\footnotetext{
${ }^{1}$ All that follows is a very straightforward extension of the standard theory of Fourier transform. Further details were presented in the Appendix $\mathrm{C}$ of [Mo08] where we used complex coordinates $(\zeta, \bar{\zeta})$ on the sphere instead of our $(\theta, \varphi)$, but this affects by no means the definitions and results.
} 
arising from the boundary to the bulk counterpart. In [DMP08], we tackled this problem showing that it is possible to realize $\left(\mathcal{S}(M), \sigma_{M}\right)$ as a subspace of $\left(\mathcal{S}\left(\Im^{-}\right), \sigma_{\Im^{-}}\right)$by means of an injective symplectomorphism $\mathcal{S}(M) \rightarrow \mathcal{S}\left(\Im^{-}\right)$. This result, in turn, implies the existence of an identification, $\imath: \mathcal{W}(M) \rightarrow \mathcal{W}\left(\Im^{-}\right)$, of the algebra of bulk observables $\mathcal{W}(M)$ and a sub algebra of observables of the boundary $\mathcal{W}\left(\Im^{-}\right)$. We review the procedure showing, in theorem 2.1 below, that the result is valid also with our more general definition of $\mathcal{S}\left(\Im^{-}\right)$and referring to a large class of values of $\nu$ which includes the most physically interesting ones as stressed in (1) of remark 2.1.

We start by reminding that any of the spacetimes $M$ we are considering can be extended to a second spacetime $\widehat{M}$ which both is globally hyperbolic in its own right and it includes $\Im^{-}$as a null hypersurface [DMP08]. By a standard argument (see [BS06] for the general case), outside the support of Cauchy data of $\Phi$, one can deform the employed Cauchy surface of $M$ to a Cauchy surface of $\widehat{M}$. Since $P$ is a second-order hyperbolic partial differential operator and it can be extended in the analogy for $\widehat{M}$, a unique solution $\Phi^{\prime}$ of (5) exists in $\widehat{M}$ with the same initial compactly supported data as those of $\Phi$. By uniqueness $\Phi^{\prime} \uparrow_{M}=\Phi$. Furthermore, since also $\Im^{-} \subset \widehat{M}$, we can define the linear map:

$$
-H^{-1} \Gamma: \mathcal{S}(M) \rightarrow C^{\infty}\left(\Im^{-} ; \mathbb{R}\right) \quad \text { such that } \Gamma(\Phi) \doteq \Phi^{\prime} \Im_{\Im^{-}} .
$$

However such a result does not guarantee a sufficient regularity of the image on $\Im^{-}$of the solution of (5) in order that $\Gamma(\Phi) \in \mathcal{S}\left(\Im^{-}\right)$; therefore, we shall analyse more in detail the structure of $\Phi$ itself. The following technical proposition establishes in fact that this is the case. It is based on the following observation. With the same procedure preformed in the proof of theorem 4.4 in [DMP08], employing the estimate stated in (3) of remark 2.1, one gets that:

$$
-\frac{1}{H}(\Gamma \Phi)(\ell, \theta, \varphi)=i H e^{-i \frac{\pi}{4}} \int_{0}^{\infty} \frac{e^{-i \ell k}}{\sqrt{2 \pi}} \sqrt{\frac{k H}{2}} \widetilde{\Phi}(H k, \eta(\theta, \varphi)) d k+\text { c.c. }
$$

where $\widetilde{\Phi}$ coincides with $(15)$ written in suitable spherical coordinates $(i . e ., \widetilde{\Phi}(\mathbf{u})=\widetilde{\Phi}(|\mathbf{u}|, \vartheta, \phi)$, with $(\vartheta, \phi)$ polar angles of $\mathbf{u})$ and $\eta:(\theta, \varphi) \mapsto(\pi-\theta, \varphi+2 \pi)$ the parity inversion.

Proposition 2.1. $\quad$ Assume that $\xi$ and $m$ are such that either $\nu$ in (11) satisfies Re $<3 / 2$, and $V(\tau)=O\left(1 / \tau^{5}\right)$ or $\nu$ in (11) satisfies Re $<1 / 2$ and $V(\tau)=O\left(1 / \tau^{3}\right)$. If $\Phi \in \mathcal{S}(M)$, the following facts hold for $0<\epsilon<3 / 2-$ Re $\nu$.

(a) $\Gamma \Phi$ decays faster than $1 / \ell^{\epsilon}$ uniformly in the angular variables.

(b) $\partial_{\ell} \Gamma \Phi$ decays faster than $1 / \ell^{1+\epsilon}$ uniformly in the angular variables.

(c) $\Gamma \Phi \in \mathcal{S}\left(\Im^{-}\right)$.

(d) Particularly, referring to the Fourier transform $\widehat{\Gamma \Phi}$ with $\Phi \in \mathcal{S}(M)$, it holds $\widehat{\Gamma \Phi} \in C^{\infty}((\mathbb{R} \backslash\{0\}) \times$ $\left.\mathbb{S}^{2} ; \mathbb{C}\right)$, it vanishes uniformly in the angles faster than every negative power of $k$ as $|k| \rightarrow+\infty$,

$$
\left|\partial_{k}^{n} \widehat{\Gamma \Phi}(k, \omega)\right| \leq C_{\delta, n} /|k|^{R e \nu+n-1 / 2}, \quad \text { for } 0<|k| \leq \delta,
$$

if $n=0,1,2,3$ and for some $C_{\delta, n}, \delta>0$.

Proof. Let us start with (b) taking (23) into account as well as (3) in remark 2.1. Without losing generality, let us assume that $\ell$ is positive then

$$
\left|\ell^{1+\epsilon} \partial_{\ell}(\Gamma \Phi)(\ell, \omega)\right| \leq \sup _{\omega^{\prime} \in \mathbb{S}^{2}}|\ell|^{\epsilon}\left|\int_{-\infty}^{+\infty} d k e^{-i k \ell} \frac{1+|k \ell|}{1+|k \ell|} \partial_{k}\left[i k \sqrt{\frac{k H}{2}} \widetilde{\Phi}\left(H k, \eta\left(\omega^{\prime}\right)\right)\right]\right| .
$$


The right-hand side could in principle diverge, but we are going to show that, indeed, this is not the case. Taking $\Psi(k, \omega)$ as a shorter notation for $\partial_{k}\left[i k \sqrt{\frac{k H}{2}} \widetilde{\Phi}(H k, \eta(\omega))\right]$ we get

$$
\left|\ell^{1+\epsilon} \partial_{\ell}(\Gamma \Phi)(\ell, \omega)\right| \leq \sup _{\mathbb{S}^{2}}|\ell|^{\epsilon}\left|\int_{-\infty}^{+\infty} d k \frac{e^{-i k \ell}+i|k| \partial_{k} e^{-i k \ell}}{1+|k \ell|} \Psi\right|
$$

Integrating by parts using (16), the preceding expression can be rewritten as:

$$
\left|\ell^{1+\epsilon} \partial_{\ell}(\Gamma \Phi)(\ell, \omega)\right| \leq \sup _{\mathbb{S}^{2}}|\ell|^{\epsilon}\left|\int_{-\infty}^{+\infty} \frac{e^{-i k \ell}}{1+|k \ell|}\left[\left(1-i\left(\frac{\sigma(k)}{1+|k \ell|}\right)\right) \Psi-i|k| \partial_{k} \Psi\right]\right|,
$$

where $\sigma(k)=1$ for $k \geq 0$ or -1 otherwise. Now let us assume $\epsilon<1$. With this choice $|k \ell|^{\epsilon} /(1+|k \ell|) \leq 1$, so that we obtain the following estimate:

$$
\left|\ell^{1+\epsilon} \partial_{\ell}(\Gamma \Phi)(\ell, \omega)\right| \leq \sup _{\mathbb{S}^{2}} \int_{-\infty}^{+\infty} d k \frac{1}{|k|^{\epsilon}}\left[2|\Psi|+\left|k \partial_{k} \Psi\right|\right]
$$

which is meaningful because the right-hand side is finite and it does not depend on the angles since both the functions in the integral can be bounded by $L^{1}\left(\mathbb{R},|k|^{-\epsilon} d k\right)$ functions (independent form angles), in view of (17) and (16). We have established the $(\theta, \varphi)$-uniform bound:

$$
\left|\partial_{\ell}(\Gamma \Phi)(\ell, \theta, \varphi)\right| \leq \frac{C}{|\ell|^{1+\epsilon}}
$$

for some constant $C>0$. If $\epsilon \geq 1$, one starts with the inequality

$$
\left|\ell^{1+\epsilon} \partial_{\ell}(\Gamma \Phi)(\ell, \omega)\right| \leq \sup _{\omega^{\prime} \in \mathbb{S}^{2}}|\ell|^{\epsilon-1}\left|\int_{-\infty}^{+\infty} d k e^{-i k \ell} \frac{1+|k \ell|}{1+|k \ell|} \partial_{k}^{2}\left[i k \sqrt{\frac{k H}{2}} \widetilde{\Phi}\left(H k, \eta\left(\omega^{\prime}\right)\right)\right]\right| ;
$$

then the proof goes on as before since $\epsilon-1<1$. (a) can be similarly proved. (c) We have obtained that $\Gamma \Phi$ is bounded (it being everywhere continuous and vanishing at infinity uniformly in the angles). Similarly, in view of Fubini-Tonelli theorem and on the fact that $\mathbb{S}^{2}$ has finite measure, $\partial_{\ell} \Gamma \Phi \in L^{1}\left(\mathbb{R} \times \mathbb{S}^{2} ; d \ell d S^{2}\right)$ since it is continuous (so that it is bounded on compact sets $[-L, L] \times \mathbb{S}^{2}$ and decays faster than $1 / \ell^{1+\epsilon}$ uniformly in the angles outside $[-L, L]$. As the requirement $\|k \widehat{\Gamma \Phi}\|_{\infty}<\infty$ is trivially fulfilled by (3) in remark 2.1, to conclude the proof of (c) it is enough to establish that the Fourier transform of $\Gamma \Phi$ belongs to $L^{1}\left(\mathbb{R}_{+} \times \mathbb{S}^{2}, d k d S^{2}(\theta, \varphi)\right)$. Since, as we have stated in (3) of remark $2.1, \widetilde{\Phi}$ is rapidly decreasing at infinity as a function of $k$, the functions $k^{1 / 2} \widetilde{\Phi}$ which is proportional to the Fourier transform of $\Gamma \Phi$ belongs to $L^{1}\left(\mathbb{R}_{+} \times \mathbb{S}^{2}, d k d S^{2}(\theta, \varphi)\right)$ in our hypotheses. We conclude that $\Gamma \Phi \in \mathcal{S}\left(\Im^{-}\right)$.

The statement (d) follows immediately from (23) and from the estimates in (3) of remark 2.1.

Remark 2.2. It is worth noticing that the result was achieved thanks to the very definition of $\mathcal{S}\left(\Im^{-}\right)$. With the stricter definition of $\mathcal{S}\left(\Im^{-}\right)$adopted in [DMP08], where the functions are required to be $L^{2}\left(\mathbb{R} \times \mathbb{S}^{2}, d \ell d S^{2}\right)$ together with their $\ell$-derivative, the above result would have been much more difficult, if not impossible, to establish.

We are now in place to state the theorem which establishes that $\Gamma$ individuates a symplectomorphism. This entails the identification between the bulk algebra of observables and a subalgebra of the boundary 
counterpart, hence extending one of the main achievements of [DMP08], namely the theorem 4.4, to the physically relevant scenario $\operatorname{Re} \nu<3 / 2$.

Theorem 2.1. Assume that $\xi$ and $m$ are such that $\nu$ in (11) satisfies $\operatorname{Re} \nu<3 / 2$, and that $V(\tau)=$ $O\left(1 / \tau^{5}\right)$ or $\nu$ in (11) satisfies Re $<1 / 2$, and that $V(\tau)=O\left(1 / \tau^{3}\right)$. The following holds.

(a) The linear map $-H^{-1} \Gamma: \mathcal{S}(M) \rightarrow C^{\infty}\left(\Im^{-} ; \mathbb{R}\right)$ is a symplectomorphism:

$$
\sigma_{\Im^{-}}\left(-H^{-1} \Gamma \Phi,-H^{-1} \Gamma \Phi^{\prime}\right)=\sigma_{M}\left(\Phi, \Phi^{\prime}\right), \quad \forall \Phi, \Phi^{\prime} \in \mathcal{S}(M)
$$

and $-H^{-1} \Gamma$ is injective.

(b) There is an isometric $*$-homomorphism

$$
\imath: \mathcal{W}(M) \rightarrow \mathcal{W}\left(\Im^{-}\right)
$$

which identifies the Weyl algebra of the observables $\mathcal{W}(M)$ of the bulk with a sub $C^{*}$-algebra of $\mathcal{W}\left(\Im^{-}\right)$, and $\imath$ is completely individuated by the requirement

$$
\imath\left(W_{M}(\Phi)\right)=W_{\Im^{-}}\left(-H^{-1} \Gamma \Phi\right) . \quad \forall \Phi \in \mathcal{S}(M)
$$

Proof. (a) By direct inspection, if $\Phi, \Phi^{\prime} \in \mathcal{S}(M)$ and making use of (8) one almost immediately gets

$$
-2 \operatorname{Im} \int_{\mathbb{R}_{+} \times \mathbb{S}^{2}} \overline{\widetilde{\Phi}(k, \theta, \varphi)} \widetilde{\Phi}^{\prime}(k, \theta, \varphi) k^{2} d k d S^{2}(\theta, \varphi)=\sigma_{M}\left(\Phi, \Phi^{\prime}\right),
$$

where the integral makes sense because $k \widetilde{\Phi}$ and $k \widetilde{\Phi}^{\prime}$ are elements of $L^{2}\left(\mathbb{R} \times \mathbb{S}^{2}, d k d S^{2}\right)$ as follows form (d) in Proposition 2.1. On the other hand, we shall show at the end of the proof of proposition 2.2 (and such proof does not depend on this one) that

$$
\sigma_{\Im^{-}}\left(\Gamma \Phi, \Gamma \Phi^{\prime}\right)=-2 H^{2} I m \int_{\mathbb{R}_{+} \times \mathbb{S}^{2}} \overline{\widetilde{\Phi}(k, \theta, \varphi)} \widetilde{\Phi}^{\prime}(k, \theta, \varphi) k^{2} d k d S^{2}(\theta, \varphi) .
$$

This concludes the proof of (a) by comparison with the identity achieved above and noticing that the found symplectomorphism is injective because $\sigma_{M}$ is nondegenerate. (b) This fact straightforwardly follows from the existence of the symplectomorphism $-H^{-1} \Gamma$ and known theorems on Weyl algebras [BR022].

2.3. Preferred state and its pullback on $M$. The existence of the isometric $*$-homomorphism $\imath: \mathcal{W}(M) \rightarrow \mathcal{W}\left(\Im^{-}\right)$allows one to induce states $\omega_{M}$ on $\mathcal{W}(M)$ from states $\omega_{\Im^{-}}$on $\mathcal{W}\left(\Im^{-}\right)$exploiting the pull back:

$$
\omega_{M}(a) \doteq \omega_{\Im^{-}}(\imath(a)), \quad \forall a \in \mathcal{W}(M) .
$$

The most distinguished property displayed by the quantum theory on the null surface $\Im^{-}$(and this is also true for the theory in any null infinity of asymptotically flat spacetime) is the following [DMP06, DMP08]. It is possible to select a preferred algebraic quasifree state $\lambda$ on $\mathcal{W}\left(\Im^{-}\right)$, which turns out to be invariant under the action of the conformal Killing vector $\partial_{\ell}$ and it has positive energy with respect to the selfadjoint generator of those displacements in its GNS representation. These features uniquely individuate 
the state [Mo06, DMP08]. In the general context studied in [DMP08], one sees that $\lambda$ is invariant under the whole, infinite dimensional, group $S G_{\Im^{-}}$of symmetries of $\Im^{-}$(see remark 1.1) and this property is valid, referring to the BMS group, for the analogue state defined on the null boundary of asymptotically flat spacetimes [DMP06]. The state $\lambda$ is universal and does not depend on the particular spacetime $M$ admitting $\Im^{-}$as past boundary. Using (27), $\lambda$ induces a preferred state $\lambda_{M}$ in every spacetime of the class under consideration. The very peculiar properties of those states were investigated in [Mo08] for asymptotically flat spacetimes and in [DMP08] for expanding universes. It was shown that $\lambda_{M}$ is invariant under all the isometries of $M$ (which preserve structure of $\Im^{-}$in the case of expanding universes), it has positive energy with respect to every timelike Killing vector of $M$ (which preserve structure of $\Im^{-}$ for expanding universes) and, furthermore, it reduces to well-known physically meaningful states in the simplest cases (Minkowski vacuum and Bunch-Davies, respectively). In the case of asymptotically flat spacetimes $\lambda_{M}$ was proved to be Hadamard [Mo08] and, thus, it can be employed in perturbative approaches. This is the issue we wish to examine here for our class of spacetimes.

We want now to define the preferred quasifree state $\lambda$ on $\mathcal{W}\left(\Im^{-}\right)$. The definition needs more care than in [DMP06], since the symplectic space has been changed. Following [KW91], a quasifree state $\omega$ over a Weyl algebra $\mathcal{W}(\mathcal{S})$ over the symplectic space $(\mathcal{S}, \sigma)$ is individuated by its one-particle structure, that is a pair $(K, H)$, where $H$ is the one-particle (complex) Hilbert space and $K: \mathcal{S} \rightarrow H$ is an $\mathbb{R}$-linear map such that (i) $\sigma\left(\psi, \psi^{\prime}\right)=-2 \operatorname{Im}\left\langle K \psi, K \psi^{\prime}\right\rangle$ for all $\psi, \psi^{\prime} \in \mathcal{S}$ and (ii) $\overline{K(\mathcal{S})+i K(\mathcal{S})}=H$, the bar denoting the closure. The quasifree state $\omega$ uniquely (up to unitary transformations) associated with $(K, H)$ is then completely individuated by the requirement (which extends to the whole $\mathcal{W}$ by linearity and continuity)

$$
\omega(W(\psi))=e^{-\frac{1}{2} \operatorname{Re}\langle K \psi, K \psi\rangle}, \quad \forall \psi \in \mathcal{S} .
$$

The state $\omega$ turns out to be pure (i.e., its GNS representation is irreducible) if and only if $\overline{K(\mathcal{S})}=H$. The GNS representation of a quasifree state $\omega$ is always a standard Fock representation with $H$ as oneparticle space, the cyclic vector is the vacuum and the representation itself maps $W(\psi)$ into $e^{\overline{i \hat{\Phi}(\psi)}}$ where $\hat{\Phi}$ is the densely defined field operator constructed out of the creation and annihilation operators (see [KW91, Wa94, BR022] for details).

Let us come to the preferred state $\lambda$ on $\mathcal{W}\left(\Im^{-}\right)$. Following [MP03, DMP06, DMP08], its one-particle structure $\left(K_{\lambda}, H_{\lambda}\right)$ should be made as follows. $H_{\lambda}=L^{2}\left(\mathbb{R}_{+} \times \mathbb{S}^{2}, 2 d k d S^{2}\right)$ and $K_{\lambda}: \mathcal{S}\left(\Im^{-}\right) \rightarrow H_{\lambda}$ associates $\psi \in \mathcal{S}\left(\Im^{-}\right)$with its Fourier transform $\widehat{\psi}=\widehat{\psi}(k, \omega)$ restricted to the values $k \in \mathbb{R}_{+}$. Differently from [MP03, DMP06, DMP08] where the well-posedness of the construction were guaranteed by the very definition of $\mathcal{S}\left(\Im^{-}\right)$whose elements were functions of $L^{2}\left(\mathbb{R} \times \mathbb{S}^{2} ; d \ell d S^{2}\right)$ with $\ell$-derivative in $L^{2}(\mathbb{R} \times$ $\left.\mathbb{S}^{2} ; d \ell d S^{2}\right)$, now the Fourier transform has to be interpreted in the distributional sense rather than a Fourier-Plancherel transform. In principle there is no automatic reason because, with the given definition of $\mathcal{S}\left(\Im^{-}\right)$, the restriction of $\widehat{\psi}$ to $\mathbb{R}_{+}$belongs to $L^{2}\left(\mathbb{R}_{+} \times \mathbb{S}^{2}, 2 d k d S^{2}\right)$ if $\psi \in \mathcal{S}\left(\Im^{-}\right)$nor for the condition (i) above stated to be valid. Therefore a result on the well-posedness of the construction is necessary.

Proposition 2.2. $\quad$ Let us define

$$
H_{\lambda} \doteq L^{2}\left(\mathbb{R}_{+} \times \mathbb{S}^{2}, 2 d k d S^{2}\right) \quad \text { and } \quad K_{\lambda}: \mathcal{S}\left(\Im^{-}\right) \ni \psi \mapsto \Theta \cdot \widehat{\psi} \in H_{\lambda}
$$

where $\widehat{\psi}=\widehat{\psi}(k, \omega)$ is the Fourier transform of $\psi \in \mathcal{S}\left(\Im^{-}\right)$and $\Theta(k) \doteq 0$ for $k \leq 0$ and $\Theta(k) \doteq 1$ otherwise. There is a quasifree pure state $\lambda: \mathcal{W}\left(\Im^{-}\right) \rightarrow \mathbb{C}$ whose one-particle structure is, up to unitary maps, $\left(K_{\lambda}, H_{\lambda}\right)$. More precisely:

(a) the $\mathbb{R}$-linear map $K_{\lambda}: \mathcal{S}\left(\Im^{-}\right) \rightarrow H_{\lambda}$ is well defined,

(b) $\overline{K_{\lambda}\left(\mathcal{S}\left(\Im^{-}\right)\right)}=H_{\lambda}$, the bar denoting the closure, 
(c) $\sigma_{\Im^{-}}\left(\psi, \psi^{\prime}\right)=-2 \operatorname{Im}\left\langle K_{\lambda} \psi, K_{\lambda} \psi^{\prime}\right\rangle$.

Proof. The first statement is consequence of (a), (b) and (c). Let us prove them. (a) If $\psi, \psi^{\prime} \in \mathcal{S}\left(\Im^{-}\right)$, in view of the definition $(20)$ of $\mathcal{S}\left(\Im^{-}\right)$one has that

$$
\int_{\mathbb{R}_{+} \times \mathbb{S}^{2}}\left|\overline{K_{\lambda} \psi(k, \theta, \varphi)} K_{\lambda} \psi^{\prime}(k, \omega)\right| 2 k d k d S^{2},
$$

is bounded by

$$
\int_{\mathbb{R} \times \mathbb{S}^{2}}\left|\bar{\psi}(k, \omega) \widehat{\psi}^{\prime}(k, \omega)\right| 2 k d k d S^{2}(\omega) \leq \sup _{\omega \in \mathbb{S}^{2}}|k \widehat{\psi}(k, \omega)| \int_{\mathbb{R} \times \mathbb{S}^{2}} 2\left|\widehat{\psi}^{\prime}(k, \omega)\right| d k d S^{2}(\omega)<+\infty .
$$

(b) The statement is true because $K_{\lambda}\left(\mathcal{S}\left(\Im^{-}\right)\right)$includes the set, dense in $L^{2}\left(\mathbb{R}_{+} \times \mathbb{S}^{2}, 2 d k d S^{2}\right)$, of the complex smooth function with compact support which do not intersect a neighbourhood (depending of the function) of the set $\left\{k=0, \omega \in \mathbb{S}^{2}\right\}$. Indeed, if $\phi_{0}$ is one of such functions, it can be smoothly extended in the region $k<0$ as $\phi_{0}(-k, \omega) \doteq \overline{\phi_{0}(k, \omega)}$. The resulting function has inverse Fourier transform given by a real element of $\mathscr{S}\left(\mathbb{R} \times \mathbb{S}^{2}\right)$ and thus it belongs to $\mathcal{S}\left(\Im^{-}\right)$.

(c) Let $\psi \in \mathcal{S}\left(\Im^{-}\right)$and $\widehat{\varphi_{n}} \in C_{0}^{\infty}\left(\mathbb{R} \times \mathbb{S}^{2} ; \mathbb{C}\right)$ so that $\varphi_{n}(\cdot, \omega) \in \mathscr{S}(\mathbb{R})$ and $\partial_{\ell} \psi(\cdot, \omega) \in \mathscr{S}^{\prime}(\mathbb{R})$ for every $\omega \in \mathbb{S}^{2}$. By standard properties of Fourier transform of Schwartz distributions, one has:

$$
\int_{\mathbb{R}} \varphi_{n}(\ell, \omega) \partial_{\ell} \psi(\ell, \omega) d \ell=-i \int_{\mathbb{R}} \widehat{\widehat{\varphi_{n}}(k, \omega)} \widehat{\psi}(k, \omega) k d k .
$$

However both the right-hand side and the left-hand side can be interpreted as standard integrals, in our hypotheses on $\mathcal{S}\left(\Im^{-}\right)$. Now fix $\psi^{\prime} \in \mathcal{S}\left(\Im^{-}\right)$and, taking the angles $\omega=(\theta, \varphi)$ fixed again, consider a sequence of compactly supported smooth functions $\widehat{\varphi_{n}}(k)$ which converges to $\widehat{\psi^{\prime}}(\cdot, \theta, \varphi)$ in $L^{1}(\mathbb{R}, d k)$. Notice that this implies that $\left\|\varphi_{n}(\cdot, \omega)-\psi(\cdot, \omega)\right\|_{\infty} \rightarrow 0$, by standard properties of Fourier transform. As a consequence one has from (30):

$$
\begin{aligned}
& \int_{\mathbb{R}} \psi^{\prime}(\ell, \omega) \partial_{\ell} \psi(\ell, \omega) d \ell=\lim _{n \rightarrow+\infty} \int_{\mathbb{R}} \varphi_{n}(\ell, \omega) \partial_{\ell} \psi(\ell, \omega) d \ell= \\
= & \lim _{n \rightarrow+\infty} \int_{\mathbb{R}}-i \overline{\widehat{\varphi_{n}}(k, \omega)} \widehat{\psi}(k, \omega) k d k=\int_{\mathbb{R}}-i \overline{\hat{\psi}^{\prime}(k, \omega)} \widehat{\psi}(k, \omega) k d k .
\end{aligned}
$$

Concerning the third and the first identity we have exploited the inequalities:

$$
\begin{gathered}
\int_{\mathbb{R}}\left|\overline{\varphi_{n}} \widehat{\psi}-\widehat{\bar{\psi}^{\prime}} \widehat{\psi}\right| 2 k d k \leq\|2 k \widehat{\psi}(\cdot, \omega)\|_{\infty}\left\|\widehat{\varphi_{n}}(\cdot, \omega)-\widehat{\psi^{\prime}}(\cdot, \omega)\right\|_{L^{1}} \rightarrow 0 \quad \text { as } n \rightarrow+\infty, \\
\int_{\mathbb{R}}\left|\varphi_{n} \partial_{\ell} \psi-\psi^{\prime} \partial_{\ell} \psi\right| d \ell \leq\left\|\partial_{l} \psi(\cdot, \omega)\right\|_{L^{1}}\left\|\varphi_{n}(\cdot, \omega)-\psi^{\prime}(\cdot, \omega)\right\|_{\infty} \rightarrow 0 \quad \text { as } n \rightarrow+\infty .
\end{gathered}
$$

We have obtained that

$$
\int_{\mathbb{R}} \psi^{\prime}(\ell, \omega) \partial_{\ell} \psi(\ell, \omega) d \ell=-i \int_{\mathbb{R}} \overline{\widehat{\psi}^{\prime}(k, \omega)} \widehat{\psi}(k, \omega) k d k .
$$

We know by the proof of (a) that $\overline{\widehat{\psi}^{\prime}} \widehat{\psi} \in L^{2}\left(\mathbb{R} \times \mathbb{S}^{2}, d k d S^{2}\right)$ and, by the very definition of $\mathcal{S}\left(\Im^{-}\right)$it results that $\psi^{\prime} \partial_{\ell} \psi \in L^{2}\left(\mathbb{R} \times \mathbb{S}^{2}, d \ell d S^{2}\right)$. The direct application of Fubini-Tonelli theorem to (31) yields

$$
\int_{\mathbb{R} \times \mathbb{S}^{2}} \psi^{\prime}(\ell, \omega) \partial_{\ell} \psi(\ell, \omega) d \ell d S^{2}=-i \int_{\mathbb{R} \times \mathbb{S}^{2}} \overline{\widehat{\psi}^{\prime}(k, \omega)} \widehat{\psi}(k, \omega) k d k d S^{2} .
$$


Using the fact that $\widehat{\psi}(-k, \omega)=\overline{\widehat{\psi}(k, \omega)}$ and $\widehat{\psi}^{\prime}(-k, \omega)=\overline{\widehat{\psi}^{\prime}(k, \omega)}$ because $\psi, \psi^{\prime}$ are real, (32) together with the definition of $K_{\lambda}$ and $\sigma_{\Im^{-}}$implies that $\sigma_{\Im^{-}}\left(\psi, \psi^{\prime}\right)=-2 \operatorname{Im}\left\langle K_{\lambda} \psi, K_{\lambda} \psi^{\prime}\right\rangle$, as wanted. This identity implies the validity of (26) using the fact that, from (23),

$$
K_{\lambda}\left(-H^{-1} \Gamma(\Phi)\right)=-i H e^{-i \pi / 4} \sqrt{\frac{H k}{2}} \widetilde{\Phi}(H k, \eta(\omega)),
$$

and taking into account that $-H^{-1} \Gamma(\Phi) \in \mathcal{S}\left(\Im^{-}\right)$when $\Phi \in \mathcal{S}(M)$ as proved in (a) of theorem 2.1.

We have now all the ingredients to construct a bulk state starting from $\lambda$, the boundary counterpart, proceeding as indicate in (27) at the beginning of this section. We define the quasifree state $\lambda_{M}$ on $\mathcal{W}(M)$ individuated by the requirement:

$$
\lambda_{M}(a) \doteq \lambda(\imath(a)), \quad \forall a \in \mathcal{W}(M)
$$

It is worth stressing that in [DMP08] a different definition of $\mathrm{S}\left(\Im^{-}\right)$was exploited, however, as it can be checked by direct inspection, all the above-mentioned properties of $\lambda$ and $\lambda_{M}$ can be proved with the definition given in this paper for the whole class of spacetimes (which are not homogeneous nor isotropous in general) discussed i9n [DMP08], essentially because $\lambda$ is defined employing (29) also in [DMP08] and because the the image of the symplectomorphism which associates a wavefunction in the bulk with its restriction to $\Im^{-}$is included in $\mathrm{S}\left(\Im^{+}\right)$no matter which of the two definition is adopted. In particular, if $M$ is the de Sitter spacetime, $\lambda_{M}$ is nothing but the Bunch-Davies vacuum [SS76, BD78, Al85, KiGa93] as discussed in [DMP08].

\section{On the Hadamard property.}

In this section we shall prove the main statement of the paper: (33) is of Hadamard form for every FRW spacetime in the class individuated by the metric (2) with the constraints (3) and for values of $\nu$ in (11) either such that $\operatorname{Re} \nu<1 / 2$ or such that $\operatorname{Re\nu }<3 / 2$ though requiring the shape of the scale factor to be that of (14). Let us remember that this latter request entails the potential $V$, appearing in (9), to satisfy $V(\tau)=O\left(1 / \tau^{5}\right)$.

As we stressed in the introduction, this scenario is of certain physical relevance if we think of inflation models where a scalar field with $\nu$ close or equal to $\frac{3}{2}$ is employed as the building block; remarkably the perturbative fluctuations lead to an almost homogeneous power spectrum which can be indirectly observed by experiments and a byproduct of the results of this section is to provide a mathematical consistency to the underlying employed quantisation scheme.

There are many reasons to consider Hadamard states as the most physically relevant ones and it exists a well-developed literature discussing them, especially in relation with the problem both of construction of Wick polynomials and, more generally, of renormalisation in curved spacetime [BFK96, BF00, HW01, BFV03]. Roughly speaking, the Hadamard property for a state is very important in QFT in curved spacetime because it assures that the stress energy tensor operator [Mo03, HW05] evaluated on that state is renormalizable [Wa94] and, thus, the theory might be gravitationally stable at least at perturbative level.

We shall quickly recall the main features of the notion of an Hadamard state leaving a reader interested in more details to specific papers [KW91, Rad96a] (see also [SA08] for some recent achievements). Consider a smooth globally hyperbolic spacetime $(M, g)$, let $\left(\mathcal{S}(M), \sigma_{M}\right)$ the real symplectic space of the real smooth solutions of Klein-Gordon equation with compactly supported Cauchy data, as defined previously. $E \doteq A-R: C_{0}^{\infty}(M ; \mathbb{R}) \rightarrow \mathcal{S}(M)$ the causal propagator [KW91, Wa94, BGP96] associated with 
the Klein-Gordon operator $P$ in (5). $A$ and $R$ are, respectively, the advanced and retarded fundamental solutions. $E$ it is known to be onto $\mathcal{S}(M)$, with kernel given by the functions $P f$, for all $f \in C_{0}^{\infty}(M ; \mathbb{R})$ and it is continuous as an operator from $C_{0}^{\infty}(M ; \mathbb{R})$ to $C^{\infty}(M ; \mathbb{R})$ in the relevant topologies of the considered spaces of test functions. The two-point function of a quasifree state $\omega$ over $\mathcal{W}(M)$ with one particle structure $(K, H)$ can be defined (see [KW91] for further details) as the quadratic form:

$$
\omega(f, g):=\langle K(E f), K(E g)\rangle_{H}, \quad \forall f, g \in C_{0}^{\infty}(M ; \mathbb{R}) .
$$

If $(f, g) \mapsto \omega(f, g)$ is weakly continuous in each argument separately, the Schwartz kernel theorem assures that $\omega(\cdot, \cdot)$ uniquely individuates a distribution $\omega(\cdot) \in \mathcal{D}^{\prime}(M \times M)$, known as the Schwartz kernel of $\omega(\cdot, \cdot)$, by requiring $\omega(f \otimes g)=\omega(f, g)$ for all $f, g \in C_{0}^{\infty}(M ; \mathbb{C})$. In fact, $\omega(\cdot)$ is nothing but the integral kernel $\omega(x, y)$ in a distributional sense:

$$
\omega(f, g)=\int_{M \times M} \omega(x, y) f(x) g(y) d \mu_{g}(x) d \mu_{g}(y), \quad \omega(h)=\int_{M \times M} \omega(x, y) h(x, y) d \mu_{g}(x) d \mu_{g}(y),
$$

if $f, g \in C_{0}^{\infty}(M ; \mathbb{C})$ and $\left.h \in C_{0}^{\infty}(M \times M ; \mathbb{C})\right)$ and $d \mu_{g}$ denoting the metric-induced measure on $M$. In the following we shall use the same symbol to denote a quasifree state, the associated quadratic form and its Schwartz kernel when the meaning of the symbol will be clear from the context.

A quasifree state $\omega$ over $\mathcal{W}(M)$ is Hadamard if its kernel $\omega(x, y)$ enjoys a very peculiar behaviour at short distance of the arguments. We shall not enter into details here [KW91] because we shall deal with the microlocal characterisation of Hadamard states due to Radzikowski [Rad96a, Rad96b].

Proposition 3.1. In a globally hyperbolic spacetime $(M, g)$, consider a quasi-free state $\omega$ for the real smooth Klein Gordon field. Assume that the two-point function of the state individuates a distribution in $\mathcal{D}^{\prime}(M \times M)$. The state $\omega$ is Hadamard if and only if the wavefront set $W F(\omega)$ of the Schwartz kernel of the two-point function has the form:

$$
W F(\omega)=\left\{\left(\left(x, k_{x}\right),\left(y,-k_{y}\right)\right) \in\left(T^{*} M\right)^{2} \backslash 0 \mid\left(x, k_{x}\right) \sim\left(y, k_{y}\right), k_{x} \triangleright 0\right\},
$$

where $\left(x, k_{x}\right) \sim\left(y, k_{y}\right)$ means that it exists a null geodesic connecting $x$ and $y$ with cotangent vectors respectively $k_{x}$ and $k_{y}$, whereas $k_{x} \triangleright 0$ means the $k_{x}$ is causal and future-directed. Here 0 is the zero section in the cotangent bundle.

In [Mo08], it was proved that the analogue of the state $\lambda_{M}$ introduced in [DMP06] for asymptotically flat spacetimes at null infinity is Hadamard. A similar proof can be found in [Ho00] in a very different physical context. The main goal of this paper is to prove the Hadamard property for the states $\lambda_{M}$ in the considered class of FRW expanding universes admitting a past cosmological horizon. We state this result formally. The proof will take all the remaining part of this section and it will be divided in several technical steps.

Theorem 3.1. $\quad$ Consider a FRW spacetime $\left(M, g_{F R W}\right)$ in the class individuated by the metric (2) with the constraints (3) and for values of $\nu$ in (11) fulfilling either that Re $<1 / 2$ or that Re $<3 / 2$ though under the assumption (14). The quasifree state $\lambda_{M}$ defined on $\mathcal{W}(M)$ in (33) is Hadamard since its two-point function individuates a distribution of $\mathcal{D}^{\prime}(M \times M)$ with wavefront set:

$$
W F\left(\lambda_{M}\right)=\left\{\left(\left(x, k_{x}\right),\left(y,-k_{y}\right)\right) \in\left(T^{*} M\right)^{2} \backslash 0 \mid\left(x, k_{x}\right) \sim\left(y, k_{y}\right), k_{x} \triangleright 0\right\} .
$$


The proof of theorem 3.1 will be the topic of the rest of the paper.

\subsection{The two-point function of $\lambda_{M}$ individuates a distribution in $\mathcal{D}^{\prime}(M \times M)$.}

As the title itself suggests, we shall now dwell into the first part of the proof of Th.3.1. To start with we need the following proposition:

Proposition 3.2. With the hypotheses of Theorem 3.1, the following facts hold for the two-point function $\lambda_{M}(f, g)$.

(a) The two-point function of $\lambda_{M}$ uniquely individuates a distribution of $\mathcal{D}^{\prime}(M \times M)$, this is the Schwartz kernel associated with the quadratic form:

$$
\lambda_{M}(f, g)=\int_{\mathbb{R} \times \mathbb{S}^{2}} 2 k \Theta(k) \overline{\widehat{\psi}_{f}(k, \theta, \varphi)} \widehat{\psi}_{g}(k, \theta, \varphi) d k d S^{2}(\theta, \varphi),
$$

where $\Theta(k) \doteq 0$ if $k \leq 0$ and $\Theta(k) \doteq 0$ otherwise, $\widehat{\psi_{h}}$ is the $\ell$-Fourier transform of $\psi_{h} \doteq-H^{-1} \Gamma(E h)$, for every $h \in C_{0}^{\infty}(M ; \mathbb{R})$, where $\Gamma$ is defined as in $(22)$ and with $E: C_{0}^{\infty}(M ; \mathbb{R}) \rightarrow \mathcal{S}(M)$ denoting the causal propagator of the Klein-Gordon operator $P$ in (5).

(b) Referring to the frame $(\ell, \theta, \varphi)$ on $\Im^{-}$, if $\operatorname{Re\nu }<1$, it holds:

$$
\lambda_{M}(f, g)=\lim _{\epsilon \rightarrow 0^{+}}-\frac{1}{\pi} \int_{\mathbb{R}^{2} \times \mathbb{S}^{2}} \frac{\psi_{f}(\ell, \theta, \varphi) \psi_{g}\left(\ell^{\prime}, \theta, \varphi\right)}{\left(\ell-\ell^{\prime}-i \epsilon\right)^{2}} d \ell d \ell^{\prime} d S^{2}(\theta, \varphi) .
$$

Proof of Theorem 3.2. (a) Let us consider two solutions $\phi_{f}=E f$ and $\phi_{g}=E g$ of (5) associated with any two functions $f, g \in C_{0}^{\infty}(M ; \mathbb{R})$. Define $\psi_{f} \doteq-H^{-1} \Gamma \phi_{f}$ and $\psi_{g} \doteq-H^{-1} \Gamma \phi_{g}$. In view of Theorem 2.2 and the definition of the state $\lambda_{M}$, we have:

$$
\lambda_{M}(f, g)=\int_{\mathbb{R} \times \mathbb{S}^{2}} 2 k \Theta(k) \overline{\widehat{\psi}_{f}}(k, \theta, \varphi) \widehat{\psi}_{g}(k, \theta, \varphi) d k d S^{2}(\theta, \varphi),
$$

where $\Theta(k) \doteq 0$ if $k \leq 0$ and $\Theta(k) \doteq 0$ otherwise. We postpone the proof of (a) at the end of the proof of the statement (b).

(b) Let us show that (36) is equivalent to (37) if $\operatorname{Re} \nu<1$. At fixed angles $\omega=(\theta, \varphi)$, we consider a sequence of real compactly-supported smooth functions $\varphi_{n}$ whose $\ell$-Fourier transform $\widehat{\varphi_{n}}$ converge to $\widehat{\psi_{g}}$ in the $L^{1}(\mathbb{R}, d k)$ norm. Since $k \widehat{\psi_{f}} \in L^{1}(\mathbb{R}, k d k)$ by definition of $\mathcal{S}\left(\Im^{-}\right)$and the $\widehat{\varphi_{n}}$ are bounded, we obtain via Lebesgue's dominated convergence:

$$
\int_{\mathbb{R}} 2 k \Theta(k) \overline{\widehat{\psi}_{f}}(k, \omega) \widehat{\varphi_{n}}(k, \omega) d k=\lim _{\epsilon \rightarrow 0^{+}} \int_{0}^{\infty} e^{-\epsilon k} 2 k \overline{\psi_{f}}(k, \omega) \widehat{\varphi_{n}}(k, \omega) d k
$$

Now notice that both $k \mapsto 2 k e^{-\epsilon k} \Theta(k) \widehat{\psi}_{f}(k, \omega)$ and $k \mapsto \widehat{\varphi_{n}}(k, \omega)$ are functions of $L^{2}(\mathbb{R}, d k)$, the former because of $(\mathrm{d})$ in Proposition 2.1. Therefore the Fourier transform can be interpreted as the FourierPlancherel one - say $\mathscr{F}$ - and

$$
\left\langle 2 k e^{-\epsilon k} \Theta \widehat{\psi}_{f}, \widehat{\varphi}_{n}\right\rangle_{L^{2}(\mathbb{R}, d k)}=\left\langle\mathscr{F}^{-1}\left(2 k \Theta e^{-\epsilon k} \widehat{\psi}_{g}\right), \varphi_{n}\right\rangle_{L^{2}(\mathbb{R}, d k)},
$$

We can now use the convolution theorem in $L^{2}(\mathbb{R}, d k)$ to rearrange the right-hand side of the internal product as:

$$
\mathcal{F}^{-1}\left(\Theta e^{-\epsilon k} \widehat{\psi}_{f}\right)(\ell, \omega)=\frac{1}{\pi} \int_{\mathbb{R}} \frac{\partial_{\ell^{\prime}} \psi_{f}\left(\ell^{\prime}, \theta, \varphi\right)}{\left(\ell-\ell^{\prime}-i \epsilon\right)} d \ell^{\prime},
$$


With this in mind we have that:

$$
\left\langle 2 k e^{-\epsilon k} \Theta \widehat{\psi}_{f}, \widehat{\varphi_{n}}\right\rangle_{L^{2}(\mathbb{R}, d k)}=-\frac{1}{\pi} \int_{\mathbb{R}} d \ell \varphi_{n}(\ell, \omega) \int_{\mathbb{R}} d \ell^{\prime} \frac{\psi_{f}\left(\ell^{\prime}, \omega\right)}{\left(\ell-\ell^{\prime}-i \epsilon\right)^{2}}
$$

where in the last equality we integrated by parts. Using the fact that that, uniformly in the angles $\omega, \psi_{f}$ is bounded and tends to 0 as $1 /|\ell|^{\delta}$ with $\delta \in(0,3 / 2-R e \nu$ ) (see proposition 2.1 where $\delta$ was indicated by $\epsilon$ ), one sees by direct computation that, for $h \equiv 1$ or $h=\varphi_{n}$ or $h=\psi_{g}$ and where $C \geq 0$ does not depend on angles:

$$
\int_{\mathbb{R} \times \mathbb{R}} d \ell d \ell^{\prime}\left|h \frac{\psi_{f}\left(\ell^{\prime}, \omega\right)}{\left(\ell-\ell^{\prime}-i \epsilon\right)^{2}}\right| \leq\|h(\cdot, \omega)\|_{\infty} \int_{\mathbb{R} \times \mathbb{R}} d \ell d \ell^{\prime}\left|\frac{C}{\left(1+|\ell|^{\delta}\right)\left(\ell-\ell^{\prime}-i \epsilon\right)^{2}}\right|<+\infty,
$$

when one chooses $\delta>1 / 2$, and this is possible when $R e \nu<1$. Particularly this implies that, in view of Fubini-Tonelli theorem, the integrals:

$$
\int_{\mathbb{R} \times \mathbb{R}} d \ell d \ell^{\prime} \frac{\varphi_{n}(\ell, \omega) \psi_{f}\left(\ell^{\prime}, \omega\right)}{\left(\ell-\ell^{\prime}-i \epsilon\right)^{2}}=\int_{\mathbb{R}} d \ell \int_{\mathbb{R}} d \ell^{\prime} \frac{\varphi_{n}(\ell, \omega) \psi_{f}\left(\ell^{\prime}, \omega\right)}{\left(\ell-\ell^{\prime}-i \epsilon\right)^{2}},
$$

and

$$
\int_{\mathbb{R} \times \mathbb{R}} d \ell d \ell^{\prime} \frac{\psi_{g}(\ell, \omega) \psi_{f}\left(\ell^{\prime}, \omega\right)}{\left(\ell-\ell^{\prime}-i \epsilon\right)^{2}}=\int_{\mathbb{R}} d \ell \int_{\mathbb{R}} d \ell^{\prime} \frac{\psi_{g}(\ell, \omega) \psi_{f}\left(\ell^{\prime}, \omega\right)}{\left(\ell-\ell^{\prime}-i \epsilon\right)^{2}},
$$

are meaningful and the end point is

$$
\left\langle 2 k e^{-\epsilon k} \Theta \widehat{\psi}_{f}, \widehat{\varphi}_{n}\right\rangle_{L^{2}(\mathbb{R}, d k)}=-\frac{1}{\pi} \int_{\mathbb{R} \times \mathbb{R}} d \ell d \ell^{\prime} \frac{\varphi_{n}(\ell, \omega) \psi_{f}\left(\ell^{\prime}, \omega\right)}{\left(\ell-\ell^{\prime}-i \epsilon\right)^{2}} .
$$

On the other and, since $\left\|\left(\psi_{g}(\cdot, \omega)-\varphi_{n}(\cdot, \omega)\right)\right\|_{\infty} \rightarrow 0$ as $n \rightarrow+\infty$ because $\widehat{\varphi_{n}}$ converge to $\widehat{\psi_{g}}$ in the $L^{1}(\mathbb{R}, d k)$, for $n \rightarrow+\infty$ we have:

$$
\left|\int_{\mathbb{R}} d \ell \int_{\mathbb{R}} d \ell^{\prime} \frac{\left(\psi_{g}-\varphi_{n}\right) \psi_{f}\left(\ell^{\prime}, \omega\right)}{\left(\ell-\ell^{\prime}-i \epsilon\right)^{2}}\right| \leq\left\|\left(\psi_{g}-\varphi_{n}\right)\right\|_{\infty} \int_{\mathbb{R}} d \ell^{\prime}\left|\frac{\psi_{f}\left(\ell^{\prime}, \omega\right)}{\left(\ell-\ell^{\prime}-i \epsilon\right)^{2}}\right| \rightarrow 0
$$

so that, as $k \mapsto 2 k e^{-\epsilon k} \Theta(k) \widehat{\psi}_{f}(k, \omega)$ is bounded,

$$
\left\langle 2 k e^{-\epsilon k} \Theta \widehat{\psi}_{f}, \widehat{\psi}_{g}\right\rangle_{L^{2}}=\lim _{n \rightarrow+\infty}\left\langle 2 k e^{-\epsilon k} \Theta \widehat{\psi}_{f}, \widehat{\varphi}_{n}\right\rangle_{L^{2}}=-\frac{1}{\pi} \int_{\mathbb{R}^{2}} \frac{\psi_{f}(\ell, \omega) \psi_{g}\left(\ell^{\prime}, \omega\right)}{\left(\ell-\ell^{\prime}-i \epsilon\right)^{2}} d \ell d \ell^{\prime},
$$

that is

$$
\int_{0}^{\infty} e^{-\epsilon k} 2 k \overline{\widehat{\psi_{f}}(k, \omega)} \widehat{\psi_{g}}(k, \omega) d k=-\frac{1}{\pi} \int_{\mathbb{R}^{2}} \frac{\psi_{f}(\ell, \omega) \psi_{g}\left(\ell^{\prime}, \omega\right)}{\left(\ell-\ell^{\prime}-i \epsilon\right)^{2}} d \ell d \ell^{\prime} .
$$

Integrating $\omega$ over the compact set $\mathbb{S}^{2}$ (and this is possible concerning the left-hand side because the integrand belong to $L^{1}\left(\mathbb{R} \times \mathbb{S}^{2}, 2 k d k d S^{2}\right)$ in view of the definition of $\mathcal{S}\left(\Im^{-}\right)$, whereas (39) holds for the integrand in the right-hand side), it arises

$$
\int_{\mathbb{R} \times \mathbb{S}^{2}} e^{-\epsilon k} 2 k \widehat{\widehat{\psi_{f}}(k, \omega)} \widehat{\psi_{g}}(k, \omega) d k d S(\omega)=-\frac{1}{\pi} \int_{\mathbb{R}^{2} \times \mathbb{S}^{2}} \frac{\psi_{f}(\ell, \omega) \psi_{g}\left(\ell^{\prime}, \omega\right)}{\left(\ell-\ell^{\prime}-i \epsilon\right)^{2}} d \ell d \ell^{\prime} d S^{2}(\omega) .
$$


Lebesgue's dominated convergence theorem produces immediately (37) when (36) is assumed.

We conclude now the proof of the statement (a), proving that the two-point function of $\lambda_{M}$ individuates a distribution in $\mathcal{D}^{\prime}(M \times M)$. To this end we are going to show that, for any fixed $f \in C_{0}^{\infty}(M ; \mathbb{R}), \lambda_{M}(f, \cdot)$ is the weak limit of a sequence of distributions $R_{f, n} \in \mathcal{D}^{\prime}(M)$ and, for any fixed $g \in C_{0}^{\infty}(M ; \mathbb{R}), \lambda_{M}(\cdot, g)$ is the weak limit of a sequence of distributions $L_{g, n} \in \mathcal{D}^{\prime}(M)$. This fact implies that $\lambda_{M}(f, \cdot), \lambda_{M}(\cdot, g) \in$ $\mathcal{D}^{\prime}(M)$ and the map $C_{0}^{\infty}(M ; \mathbb{R}) \ni f \mapsto \lambda_{M}(f, \cdot) \in \mathcal{D}^{\prime}(M)$ is well-defined and sequentially continuous in particular. The standard argument based on Schwartz' integral kernel theorem finally implies that $\lambda_{M}(\cdot, \cdot) \in \mathcal{D}^{\prime}(M \times M)$.

The required sequences of distributions are defined as $R_{f, n}(g) \doteq \lambda_{n}(f, g)$ and $L_{g, n}(f) \doteq \lambda_{n}(f, g)$ where:

$$
\lambda_{n}(f, g) \doteq \lim _{\epsilon \rightarrow 0^{+}} \int_{\mathbb{R}^{2} \times \mathbb{S}^{2}} \frac{\left(\psi_{f} \chi_{n}\right)\left(\ell^{\prime}, \theta, \varphi\right)\left(\psi_{g} \chi_{n}\right)(\ell, \theta, \varphi)}{\left(\ell-\ell^{\prime}-i \epsilon\right)^{2}} d \ell d \ell^{\prime} d S^{2}
$$

Above $\chi_{n}(\ell) \doteq \chi(\ell / n), n=1,2, \ldots$ are some cutoff functions on $\mathbb{R} \times \mathbb{S}^{2}$ which are constant in the angular variables; they are defined out of $\chi \in C_{0}^{\infty}(\mathbb{R} ; \mathbb{R})$, such that $\chi(0)=1$. The functionals $R_{f, n}$ and $L_{g, n}$ are distributions because $\lambda_{n} \in \mathcal{D}^{\prime}(M \times M)$ since:

$$
\begin{gathered}
\left|\lambda_{n}(f, g)\right| \leq C_{n} \sum_{|m|<N} \sup \left|\partial^{m}\left(\chi_{n} \psi_{f}\right)\right| \sum_{\left|m^{\prime}\right|<N^{\prime}} \sup \left|\partial^{m^{\prime}}\left(\chi_{n} \psi_{g}\right)\right| \\
\leq C_{n} \sum_{|m|<N} \sup _{\text {supp } \chi_{n}}\left|\partial^{m}\left(\chi_{n} \psi_{f}\right)\right| \sum_{\left|m^{\prime}\right|<N^{\prime}} \sup _{\text {supp } \chi_{n}}\left|\partial^{m^{\prime}}\left(\chi_{n} \psi_{g}\right)\right| \leq C_{n}^{\prime} \sum_{|p|<M^{\prime}} \sup \left|\partial^{p} f\right| \sum_{\left|p^{\prime}\right|<M^{\prime}} \sup \left|\partial^{p^{\prime}} g\right| .
\end{gathered}
$$

The first estimate holds because the kernel $1 /\left(\ell-\ell^{\prime}-i 0^{+}\right)^{2}$ is a well-defined distribution of the space $\left.\mathcal{D}^{\prime}\left(\left(\mathbb{R} \times \mathbb{S}^{2}\right) \times\left(\mathbb{R} \times \mathbb{S}^{2}\right)\right)\right)$. In the last estimate we have used the fact that $\chi_{n} \psi_{f}=\chi_{n} E f$ where supp $\chi_{n}$ is compact, and the continuity of the causal propagator $E: C_{0}^{\infty}(M, \mathbb{R}) \rightarrow C^{\infty}(M, \mathbb{R})$ with respect to the relevant topologies. To conclude the proof it is sufficient to prove that $R_{f, n} \rightarrow \lambda(f, \cdot)$ and $L_{g, n} \rightarrow \lambda(\cdot, g)$ in weak sense, as $n \rightarrow+\infty$. To this end we notice that, exploiting the proof of the part (a) in the much easier situation where $\psi_{f} \chi_{n}$ and $\psi_{g} \chi_{n}$ have compact support, one achieves:

$$
\lambda_{n}(f, g)=\int_{\mathbb{R} \times \mathbb{S}^{2}} 2 k \Theta(k) \widehat{\widehat{\chi_{n} \psi_{f}}} \widehat{\chi_{n} \psi_{g}} d k d S^{2} .
$$

Therefore, if one performs the integrals over $\mathbb{R}_{+} \times \mathbb{S}^{2}$, it holds

$$
\begin{aligned}
& \left|R_{f, n}(g)-\lambda_{M}(f, g)\right|=\left|L_{g, n}(f)-\lambda_{M}(f, g)\right|=\left|\lambda_{n}(f, g)-\lambda_{M}(f, g)\right| \\
& \leq\left|\int\left[\overline{\widehat{\chi_{n} \psi_{f}}} \widehat{\chi_{n} \psi_{g}}-\overline{\widehat{\psi_{f}}} \widehat{\psi_{g}}\right] 2 k d k d S^{2}\right| \leq \int\left[\left|\overline{\widehat{\chi_{n} \psi_{f}}} \widehat{\chi_{n} \psi_{g}}-\overline{\widehat{\chi_{n} \psi_{f}} \widehat{\psi_{g}} \mid}\right|+\left|\overline{\chi_{n} \psi_{f}} \widehat{\psi_{g}}-\overline{\widehat{\psi_{f}}} \widehat{\widehat{\psi_{g}}}\right|\right] 2 k d k d S^{2} \\
& \leq 2|| \overline{\overline{\chi_{n} \psi_{f}}}\left\|_{\infty} \int\left|\widehat{\chi_{n} \psi_{g}}-\widehat{\psi_{g}}\right| d k d S^{2}+2|| k \widehat{\psi_{g}}\right\|_{\infty} \int\left|\overline{\overline{\chi_{n} \psi_{f}}}-\overline{\widehat{\psi_{f}}}\right| d k d S^{2} .
\end{aligned}
$$

Above $k \widehat{\psi}_{g}$ is bounded by definition of $\mathcal{S}\left(\Im^{-}\right)$, and, if one makes use both of the convolution theorem for $L^{1}$ functions and of the definition of Fourier transform as well as that of $\mathcal{S}\left(\Im^{-}\right)$, one finds by direct inspection:

$$
\left|2 k \widehat{\chi_{n} \psi_{f}}\right| \leq 2(2 \pi)^{-1 / 2}\|\chi\|_{\infty}\left\|\partial_{\ell} \psi\right\|_{\infty}+2\left\|\psi_{f}\right\|_{L^{1}}(2 \pi)^{-1 / 2} \int_{\mathbb{R}}\left|\chi^{\prime}(\ell / n)\right| d(\ell / n)<C_{f}<\infty,
$$


where $C_{f}$ does not depend on $n$, though it depends on the fixed function $\chi$. We conclude that:

$\left|R_{f, n}(g)-\lambda_{M}(f, g)\right|=\left|L_{g, n}(f)-\lambda_{M}(f, g)\right| \leq C_{f} \int\left|\widehat{\chi_{n} \psi_{g}}-\widehat{\psi_{g}}\right| d k d S^{2}+\left.2|| k \widehat{\psi_{g}}\right|_{\infty} \int\left|\overline{\widehat{\chi_{n} \psi_{f}}}-\overline{\widehat{\psi_{f}}}\right| d k d S^{2}$

To conclude the proof, it is sufficient to prove that $\widehat{\chi_{n} \psi_{f}} \rightarrow \widehat{\psi}_{f}$ and $\widehat{\chi_{n} \psi_{g}} \rightarrow \widehat{\psi}_{g}$ in $L^{1}\left(\mathbb{R} \times \mathbb{S}^{2}, d k d S^{2}\right)$. To this end consider $\rho_{\delta} \in C_{0}^{\infty}(\mathbb{R} ; \mathbb{R})$ such that $\rho_{\delta}(0)=1,0 \leq|\rho(k)| \leq 1$ and $\operatorname{supp}_{\delta} \subset[-\delta, \delta]$, and define $\rho_{\delta}^{\prime} \doteq 1-\rho_{\delta}$ which is non-negative and it vanishes in a neighbourhood of $k=0$. With this definition it holds, making use of the convolution theorem in $L^{1}$

$$
\int_{\mathbb{R} \times \mathbb{S}^{2}}\left|\widehat{\chi_{n} \psi_{f}}-\widehat{\psi_{f}}\right| d k d S^{2} \leq \int_{\mathbb{R} \times \mathbb{S}^{2}}\left|\widehat{\chi_{n}} *\left(\rho_{\delta} \widehat{\psi_{f}}\right)-\rho_{\delta} \widehat{\psi_{f}}\right| d k d S^{2}+\int_{\mathbb{R} \times \mathbb{S}^{2}}\left|\widehat{\chi_{n}} *\left(\rho_{\delta}^{\prime} \widehat{\psi_{f}}\right)-\rho_{\delta}^{\prime} \widehat{\psi_{f}}\right| d k d S^{2} .
$$

On the other hand, using particularly the fact that $\widehat{\chi_{n}}(k)=n \widehat{\chi}(k n)$ and changing the coordinates in the convolution integral, one has that the first integral in the right-hand side is dominated by:

$$
\int_{\mathbb{R} \times \mathbb{S}^{2}}\left|\widehat{\chi_{n}} *\left(\rho_{\delta} \widehat{\psi_{f}}\right)\right|+\left|\rho_{\delta} \widehat{\psi_{f}}\right| d k d S^{2} \leq \int_{\mathbb{S}^{2}} d S^{2} \int_{\mathbb{R}} d h|n \widehat{\chi}(n h)| \int_{-\delta}^{\delta}\left|\widehat{\psi_{f}}(p, \omega)\right| d p+\int_{\mathbb{S}^{2}} \int_{-\delta}^{\delta}\left|\widehat{\psi_{f}}\right| d k d S^{2} .
$$

Noticing that $\left|\widehat{\psi_{f}}(k, \omega)\right| \leq B /|k|^{\epsilon}$ about $k=0$ as consequence of (d) in Proposition 2.1, we achieve the final bound, for some constant $B^{\prime} \geq 0$ independent from $\delta$ :

$$
\int_{\mathbb{R} \times \mathbb{S}^{2}}\left|\widehat{\chi_{n}} *\left(\rho_{\delta} \widehat{\psi_{f}}\right)-\rho_{\delta} \widehat{\psi_{f}}\right| d k d S^{2} \leq \int\left|\widehat{\chi_{n}} *\left(\rho_{\delta} \widehat{\psi_{f}}\right)\right|+\left|\rho_{\delta} \widehat{\psi_{f}}\right| d k d S^{2} \leq\left(4 \pi \int_{\mathbb{R}} d u|\widehat{\chi}(u)|+1\right) B^{\prime} \delta^{1-\epsilon} .
$$

Concerning the second integral in the right-hand side of (43) we observe that, as $n \rightarrow+\infty$ :

$$
\left(\widehat{\chi_{n}} *\left(\rho_{\delta}^{\prime} \widehat{\psi_{f}}\right)\right)(k, \omega)=\int_{\mathbb{R}} \widehat{\chi}(p)\left(\rho_{\delta}^{\prime} \widehat{\psi_{f}}\right)(k-p / n, \omega) d p \rightarrow \int_{\mathbb{R}} \widehat{\chi}(p) d p\left(\rho_{\delta}^{\prime} \widehat{\psi_{f}}\right)(k, \omega)=\left(\rho_{\delta}^{\prime} \widehat{\psi_{f}}\right)(k, \omega),
$$

in view of Lebesgue's dominated convergence theorem (taking into account that $\rho_{\delta}^{\prime} \widehat{\psi_{f}}$ is bounded by construction - the only singularity has been cancelled by $\rho_{\delta}^{\prime}$, and that $\left.\hat{\chi} \in L^{1}(\mathbb{R}, d k)\right)$; moreover, if one computes the inverse Fourier transform of $\left(1+k^{2}\right)\left(\widehat{\chi_{n}} *\left(\rho_{\delta}^{\prime} \widehat{\psi_{f}}\right)\right)$ taking into account both that the arguments of the convolution are Schwartz functions and that $\chi_{n}(\ell)=\chi(\ell / n)$, one sees that it can be bounded by a Schwartz function $s$ independent from $n$ and the angles, so that:

$$
\left|\left(1+k^{2}\right)\left(\widehat{\chi_{n}} *\left(\rho_{\delta}^{\prime} \widehat{\psi_{f}}\right)\right)\right| \leq \frac{1}{\sqrt{2 \pi}} \int_{\mathbb{R}}\left|e^{i k \ell} s(\ell)\right| d \ell \doteq K .
$$

Therefore there is a constant $K \geq 0$ with:

$$
\left|\left(\widehat{\chi_{n}} *\left(\rho_{\delta}^{\prime} \widehat{\psi_{f}}\right)\right)(k, \omega)\right| \leq \frac{K}{1+k^{2}},
$$

We are, thus, allowed to apply again Lebesgue's theorem to the second integral in (43), concluding that it vanishes for $n \rightarrow+\infty$,

$$
\int_{\mathbb{R} \times \mathbb{S}^{2}}\left|\widehat{\chi_{n}} *\left(\rho_{\delta}^{\prime} \widehat{\psi_{f}}\right)-\rho_{\delta}^{\prime} \widehat{\psi_{f}}\right| d k d S^{2} \rightarrow 0
$$


Summarising and focusing back on the right-hand side of (43), we can write, for every fixed $\epsilon \in(0,1)$ :

$$
\begin{gathered}
0 \leq \liminf _{n} \int_{\mathbb{R} \times \mathbb{S}^{2}}\left|\widehat{\chi_{n} \psi_{f}}-\widehat{\psi_{f}}\right| d k d S^{2} \leq \limsup _{n} \int_{\mathbb{R} \times \mathbb{S}^{2}}\left|\widehat{\chi_{n} \psi_{f}}-\widehat{\psi_{f}}\right| d k d S^{2} \leq \\
A \delta^{1-\epsilon}+\limsup _{n} \int\left|\widehat{\chi_{n}} *\left(\rho_{\delta}^{\prime} \widehat{\psi_{f}}\right)-\rho_{\delta}^{\prime} \widehat{\psi_{f}}\right| d k d S^{2}=A \delta^{1-\epsilon}+\lim _{n \rightarrow+\infty} \int\left|\widehat{\chi_{n}} *\left(\rho_{\delta}^{\prime} \widehat{\psi_{f}}\right)-\rho_{\delta}^{\prime} \widehat{\psi_{f}}\right| d k d S^{2}=A \delta^{1-\epsilon},
\end{gathered}
$$

where the constant $A \geq 0$ does not depend on $\delta>0$ which can be taken arbitrarily small. This result immediately implies that $\widehat{\chi_{n} \psi_{f}}-\widehat{\psi_{f}} \rightarrow 0$ in the topology of $L^{1}\left(\mathbb{R} \times \mathbb{S}, d k d S^{2}\right)$ and since the analogue holds for $g$, it concludes the proof of (a) and of the theorem.

\subsection{The general strategy to establish the identity (35).}

We can carry on with the proof of the theorem 3.1 proving that the wavefront set of $\lambda_{M}$ is that stated in (35). By construction, the distribution $\lambda_{M} \in \mathcal{D}^{\prime}(M \times M)$ satisfies the further properties:

$$
\lambda_{M}(f \otimes P g)=\lambda_{M}(P f \otimes g)=0 \quad(\mathbf{K G}), \quad \lambda_{M}(f \otimes g)-\lambda_{M}(g \otimes f)=E(f \otimes g) \quad(\mathbf{C o m m}),
$$

where, in the second formula, $E$ is the Schwartz kernel of the causal propagator which exists in accordance with the above-mentioned continuity properties of the causal propagator. As is well known [Rad96a, SV01, SVW02, Mo08], the inclusion $\supset$ in (35) follows from $\subset$ when one applies the celebrated theorem of propagation of singularities due to Hörmander [Hö89], in combination with (KG) and (Comm). So, only the inclusion $\subset$ has to be established. In order to prove that inclusion, we would like to interpret the $\lambda_{M}$ as a composition of distributions, though this idea will not turn out to be truly conclusive. To this end notice that, in view of Proposition 3.2, for $R e \nu<1$, the two-point function of $\lambda_{M}$ in (37) reads:

$$
\lambda_{M}(f, g)=T((\Gamma E f) \otimes(\Gamma E g)),
$$

where $\Gamma E f$ represents the restriction to $\Im^{-}$of the wave function $E f, E$ being the causal propagator, and $T$ is an integral operator whose integral kernel can be thought of as the distribution in $\mathcal{D}^{\prime}\left(\Im^{+} \times \Im^{+}\right)$:

$$
T\left(\ell, \omega, \ell^{\prime}, \omega^{\prime}\right):=-\frac{1}{H^{2} \pi^{2}\left(\ell-\ell^{\prime}-i 0^{+}\right)^{2}} \otimes \delta\left(\omega, \omega^{\prime}\right) .
$$

Above, $H$ is the Hubble constant, $\delta\left(\omega, \omega^{\prime}\right)$ is the standard delta distribution on $\mathbb{S}^{2}$ to be integrated with respect to the standard measure on the unit 2 -sphere, whereas $\ell$ is the null coordinate on $\mathbb{R}$ as in (4). Looking at (45), our strategy to establish $\subset$ in (35), concluding the proof of Theorem 3.1, will be the following. First of all we shall prove that, if $I$ is the identity from $C_{0}^{\infty}(M ; \mathbb{C}) \rightarrow C_{0}^{\infty}(M ; \mathbb{C})$ (i.e. the Schwartz kernel in $\mathcal{D}^{\prime}(M \times M)$ given by the constant function 1) the product of distributions

$$
K \doteq(T \otimes I)(\Gamma E \otimes \Gamma E) \in \mathcal{D}^{\prime}\left(\left(\Im^{-} \times \Im^{-}\right) \times(M \times M)\right)
$$

is well defined. Now notice that, if $\mathcal{K}: C_{0}^{\infty}\left(\Im^{-} \times \Im^{-} ; \mathbb{C}\right) \rightarrow \mathcal{D}^{\prime}(M \times M)$ is the continuous map associated with the kernel ${ }^{t} K$ in view of Schwartz kernel theorem, formally speaking, (45) would hold when:

$$
\lambda_{M}=\mathcal{K}(1 \otimes 1),
$$

where $1: \Im^{-} \rightarrow \mathbb{R}$ is function which takes the value 1 constantly. However that does not make sense in general because, in particular, $1 \notin C_{0}^{\infty}\left(\Im^{-} ; \mathbb{C}\right)$. As a matter of fact, we shall instead replace the function 
1 with a sequence of functions $\chi_{n} \in C_{0}^{\infty}\left(\Im^{-} ; \mathbb{C}\right)$ which tends to 1 in a suitable sense and we shall prove that, in the general case $R e \nu<3 / 2$ so that the distribution $\lambda_{M}$ is defined by (36), it holds

$$
\mathcal{K}\left(\chi_{n} \otimes \chi_{n}\right) \doteq \lambda_{n} \rightarrow \lambda_{M}, \quad \text { as } n \rightarrow+\infty,
$$

where the convergence is valid in the sense of Hörmander's pseudo topology [Hö89]. We also prove that, for every $n \in \mathbb{N}$, it holds: $W F\left(\lambda_{n}\right) \subset\left\{\left(\left(x, k_{x}\right),\left(y,-k_{y}\right)\right) \in\left(T^{*} M\right)^{2} \backslash 0 \mid\left(x, k_{x}\right) \sim\left(y, k_{y}\right), k_{x} \triangleright 0\right\}$. Taking this shape of $W F\left(\lambda_{n}\right)$ and (48) into account, the properties of the notion of convergence in Hörmander's pseudo topology will imply $\subset$ in (35), concluding the proof of Theorem 3.1.

\subsubsection{Restriction to $\Im^{-}$of $E$ and well-definiteness of $K$ in (47).}

We prove here that $K$ in (47) is a well-defined distribution given by the product of two distributions. To this end, we need a result on the extension/restriction of one entry of the causal propagator $E$ to the horizon in order to define $\Gamma E$ appearing in (47) as an element of $\mathcal{D}^{\prime}\left(\Im^{+} \times M\right)$. Next we pass to analyse $T$ and to show that the distribution $K$ is well defined.

Since both $M$ and $\widehat{M}$ are globally hyperbolic, and $M \subset \widehat{M}$, Eq.(5) admits unique causal propagators, $E$ on $M$, and $\widehat{E}$ on $\widehat{M}$. By uniqueness $\widehat{E} \uparrow_{M \times M}=E$ when both are viewed as Schwartz kernels. $\widehat{E}$ can be restricted to $\Im^{-}$in the left argument, when the other ranges in $M$, giving rise to a distribution in $\mathcal{D}^{\prime}\left(\Im^{+} \times M\right)$ we shall indicate by $\Gamma E=\Gamma E(z, x)$. Indeed, one has $[\operatorname{Rad} 96 \mathrm{a}]$ :

$$
W F(\widehat{E}):=\left\{\left(\left(z, k_{z}\right),\left(x,-k_{x}\right)\right) \in\left(T^{*} \widehat{M}\right)^{2} \backslash 0 \mid\left(z, k_{z}\right) \sim\left(x, k_{x}\right)\right\},
$$

where $\left(x, k_{x}\right) \sim\left(z, k_{z}\right)$ means that it exists a null geodesic $\gamma$ from $x$ to $z$ where $k_{x}$ is the cotangent vector in $x$ and $k_{z}$ the cotangent vector in $z$. To restrict its left entry to $\Im^{-}$, we consider the immersion map $j: \Im^{-} \times \widehat{M} \rightarrow \widehat{M} \times \widehat{M}, j(\ell, \theta, \varphi, x)=((a=0, \ell, \theta, \varphi), x)$ in the Bondi like coordinates as in (4). According to the theorem 8.2.4 of [Hö89], that restriction is meaningful provided $W F(\widehat{E}) \cap N_{j}=\emptyset$, where $N_{j}$ is the set in $T^{*} \widehat{M}$ of normals to $\Im^{-}$. In the case under investigation

$$
N_{j}:=\left\{\left(\left(z, k_{z}\right),(x, 0)\right) \in T^{*} \widehat{M} \times T^{*} \widehat{M} \mid z \in \Im^{-} x \in \widehat{M}, k_{z}=\left(k_{z}\right)_{a} d a,\left(k_{z}\right)_{a} \in \mathbb{R}\right\} .
$$

Since null structures are preserved by conformal rescaling and since (2) entails that the spacetimes under consideration are conformally related to Minkowski spacetime, the null geodesics of $\left(M, g_{F R W}\right)$ have the same causal structure as those of $\left(\mathbb{R}^{4}, \eta\right)$. If we take into account this remark in combination with the definition (49) according to which each pair of points $(x, y)$ is joined by a null geodesic, we can prove that $W F(\widehat{E}) \cap N_{j}=\emptyset$ slavishly following the proof of the analogous statement in [Mo08]. So, $\left.\Gamma E:=\widehat{E}\right\rceil_{\Im^{-} \times M}$ is well defined and Theorem 8.2.4 of [Hö89] guarantees that:

$$
W F(\Gamma E) \subset\left\{\left(\left(s, k_{s}\right),\left(x,-k_{x}\right)\right) \in\left(T^{*} \Im^{-} \times T^{*} M\right) \backslash 0 \mid\left(s, k_{s}\right) \sim\left(x, k_{x}\right),\left(k_{s}\right)_{l} \neq 0\right\} .
$$

A bound for $W F(\Gamma E \otimes \Gamma E)$ can be obtained by Theorem 8.2.9 in [Hö89], via the general formula:

$$
W F(u \otimes v) \subset(W F(u) \times W F(v)) \cup((\operatorname{supp} u \times\{0\}) \times W F(v)) \cup(W F(u) \times(\operatorname{supp} v \times\{0\})) .
$$

Let us pass to analyse $T \otimes I$. Obviously one has:

$$
W F(T \otimes I)=W F(T) \times((M \times M) \times\{0\}) .
$$

The wavefront set of $T$ was already discussed in section 4.3 of [Mo08] and we here summarise it: 
Proposition 3.3. $W F(T):=A \cup B$ where:

$$
\begin{aligned}
& A:=\left\{\left((\ell, \omega, k, \mathbf{k}),\left(\ell^{\prime}, \omega^{\prime}, k^{\prime}, \mathbf{k}^{\prime}\right)\right) \in\left(T^{*} \Im^{-}\right)^{2} \backslash 0 \mid \ell=\ell^{\prime}, \omega=\omega^{\prime}, 0<k=-k^{\prime}, \mathbf{k}=-\mathbf{k}^{\prime}\right\}, \\
& B:=\left\{\left((\ell, \omega, k, \mathbf{k}),\left(\ell^{\prime}, \omega^{\prime}, k^{\prime}, \mathbf{k}^{\prime}\right)\right) \in\left(T^{*} \Im^{-}\right)^{2} \backslash 0 \mid \omega=\omega^{\prime}, k=k^{\prime}=0, \mathbf{k}=-\mathbf{k}^{\prime}\right\},
\end{aligned}
$$

with $\ell \in \mathbb{R}, k \in T_{\ell}^{*} \mathbb{R}, \omega \in \mathbb{S}^{2}$, whereas $\mathbf{k} \in T_{\omega}^{*} \mathbb{S}^{2}$, and 0 is the zero-section of $\left(T^{*} \Im^{-}\right)^{2} \equiv T^{*}\left(\Im^{-} \times \Im^{-}\right)$.

We can conclude that $K$ in (47) is a well-defined distribution in $D^{\prime}\left(M \times M \times \Im^{-} \times \Im^{-}\right)$, since the sufficient condition for the existence of the product of two distributions, stated in term of wavefront sets in theorem 8.2.10 in [Hö89] is valid: there is no $(x, \xi) \in W F(T \otimes I)$ with $(x,-\xi) \in W F(\Gamma E \otimes \Gamma E)$. Furthermore the following inclusion holds in view of the previously cited theorem and (52):

$$
\begin{aligned}
W F(K) \subset\{ & \left(\left(s, p_{s}+\tilde{p}_{s}\right),\left(s^{\prime}, p_{s^{\prime}}+\tilde{p}_{s^{\prime}}\right),\left(x, k_{x}\right),\left(y, k_{y}\right)\right) \in T^{*} \Im^{-} \times T^{*} \Im^{-} \times T^{*} M \times T^{*} M \\
\mid & \left(\left(s, p_{s}\right),\left(s^{\prime}, p_{s^{\prime}}\right)\right) \in W F(T) \text { or } p_{s}=p_{s^{\prime}}=0, \text { and } \\
& \left.\left(\left(s, \tilde{p}_{s}\right),\left(s^{\prime}, \tilde{p}_{s^{\prime}}\right),\left(x, k_{x}\right),\left(y, k_{y}\right)\right) \in W F(\Gamma E \otimes \Gamma E) \text { or } \tilde{p}_{s}=\tilde{p}_{s^{\prime}}=k_{x}=k_{y}=0\right\} .
\end{aligned}
$$

\subsubsection{On the sequence of auxiliary distributions $\lambda_{n}$ and their wavefront set.}

To define the sequence of distributions satisfying (48), let us first fix a function $\chi \in C_{0}^{\infty}(\mathbb{R} ; \mathbb{R})$ depending on the variable $\ell$ only, and such that $\chi(0)=1$ and define $\chi_{n} \in C_{0}^{\infty}\left(\Im^{+} ; \mathbb{R}\right)$ as:

$$
\chi_{n}(\ell, \omega) \doteq \chi\left(\frac{\ell}{n}\right), \quad \text { if }(\ell, \omega) \in \mathbb{R} \times \mathbb{S}^{2}, \forall n \in \mathbb{N} .
$$

Hence we can define the following sequence of distributions, which are well defined because as proved beforehand $K \in \mathcal{D}^{\prime}\left(\left(\Im^{+} \times \Im^{+}\right) \times(M \times M)\right)$,

$$
\lambda_{n} \doteq \mathcal{K}\left(\chi_{n} \otimes \chi_{n}\right) \in \mathcal{D}^{\prime}(M \times M),
$$

where $\mathcal{K}: C_{0}^{\infty}\left(\Im^{-} \times \Im^{-} ; \mathbb{C}\right) \rightarrow \mathcal{D}^{\prime}(M \times M)$ is the continuous operator uniquely associated to ${ }^{t} K$ in accordance with Schwartz kernel theorem. These distributions have been already used in the proof of (a) of Proposition 3.2. The wavefront set of $\lambda_{n}$ satisfies the following inclusion, which can be readily inferred out of Theorem 8.2.12 in [Hö89]:

$$
W F\left(\lambda_{n}\right) \subset\left\{\left(\left(x, k_{x}\right),\left(y, k_{y}\right)\right) \mid\left((s, 0),\left(s^{\prime}, 0\right),\left(x, k_{x}\right),\left(y, k_{y}\right)\right) \in W F(K) \text {, for some } s, s^{\prime} \in \text { supp } \chi_{n}\right\} .
$$

As supp $\chi_{n}$ becomes larger and larger as $n \rightarrow+\infty$, if one wants to achieve a $n$-uniform bound of $W F\left(\lambda_{n}\right)$, the last requirement has be dropped and replaced with $s, s^{\prime} \in \Im^{-}$.

$$
W F\left(\lambda_{n}\right) \subset\left\{\left(\left(x, k_{x}\right),\left(y, k_{y}\right)\right) \mid\left((s, 0),\left(s^{\prime}, 0\right),\left(x, k_{x}\right),\left(y, k_{y}\right)\right) \in W F(K) \text {, for some } s, s^{\prime} \in \Im^{-}\right\} .
$$

Taking Proposition 3.3 and equations (50), (51) and (53) into account, with a laborious but elementary computation, this $n$-uniform estimate can be formally restated as:

Proposition 3.4. The elements $\lambda_{n} \in \mathcal{D}^{\prime}(M \times M)$ defined in (55) satisfy:

$$
W F\left(\lambda_{n}\right) \subset \mathcal{V} \doteq\left\{\left(\left(x, k_{x}\right),\left(y,-k_{y}\right)\right) \in\left(T^{*} M\right)^{2} \backslash 0 \mid\left(x, k_{x}\right) \sim\left(y, k_{y}\right), k_{x} \triangleright 0\right\} .
$$


Notice that $\mathcal{V}$ is a closed subset in $\left(T^{*} M\right)^{2} \backslash 0$. As an immediate but indirect proof, simply notice that, in view of Radzikowski's achievements, $\mathcal{V}$ is the wavefront set (and thus a closed subset in $\left(T^{*} M\right)^{2} \backslash 0$ by definition of wavefront set) of any Hadamard state on the globally hyperbolic spacetime $\left(M, g_{F} R W\right)$ (and every globally hyperbolic spacetime admits Hadamard states as is well known [Wa94]).

\subsubsection{Proof of the fact that $\lambda_{n} \rightarrow \lambda_{M}$ in $\mathcal{D}_{\mathcal{V}}^{\prime}(M \times M)$, and the consequent $W F\left(\lambda_{M}\right)$.}

In order to complete the proof of Theorem 3.1, establishing the inclusion $\subset$ in (35), we intend to show that $\left\{\lambda_{n}\right\}_{n \in \mathbb{N}}$ converges to $\lambda_{M}$ given by (36), in $\mathcal{D}_{\mathcal{V}}^{\prime}(M \times M)$ in the sense of Hörmander pseudo topology. According to the discussion after Definition 8.2.2 in [Hö89], this is equivalent to require that - without assuming a priori that $\lambda_{M} \in \mathcal{D}_{\mathcal{V}}^{\prime}(M \times M)$, but assuming that every $\lambda_{n} \in \mathcal{D}_{\mathcal{V}}^{\prime}(M \times M)-$

1. $\lambda_{n} \rightarrow \lambda_{M}$ in the topology of $\mathcal{D}^{\prime}(M \times M)$,

2. $\sup _{n} \sup _{k \in V}|k|^{N}\left|\widehat{h \lambda_{n}}\right|<\infty$ for any $N \geq 1$ and for any $h \in C_{0}^{\infty}(M \times M ; \mathbb{C})$. In this last inequality $V$ stands for any cone, closed in $\left(T^{*} M\right)^{2} \backslash 0$, in the complement of $\mathcal{V}$.

(The former requirement is a stronger version of the result achieved in the proof of (a) of Proposition (3.2) where the convergence of $\lambda_{n}$ to $\lambda$ were proved in the sense of quadratic forms only.) The reader should notice that if both the conditions written above were true, it would have to hold $\lambda_{M} \in \mathcal{D}_{\mathcal{V}}^{\prime}(M \times M)$, and this in turn implies $W F\left(\lambda_{M}\right) \subset \mathcal{V}$, a statement which is nothing but the inclusion $\subset$ in (35). Therefore the proof of the validity of both items above would conclude the proof of Theorem 3.1.

Let us establish the validity of both items separately.

Proposition 3.5. The sequence of distributions $\lambda_{n} \in \mathcal{D}^{\prime}(M \times M)$ converges to $\lambda_{M}$ in the weak sense.

Proof. We have to show that, for every $h \in C_{0}^{\infty}(M \times M ; \mathbb{C})$, it holds:

$$
\lim _{n \rightarrow \infty}\left|\lambda_{n}(h)-\lambda_{M}(h)\right|=0 .
$$

Obviously we can restrict ourselves to $h \in C_{0}^{\infty}(M \times M ; \mathbb{R})$ by linearity. In the following, we shall make use of the notations and the properties of $\Psi_{h} \doteq(\Gamma E \otimes \Gamma E) h$ and its Fourier transform given in the final part of the Appendix B. The distributions $\lambda_{n}$ acts as:

$$
\lambda_{n}(h)=\int_{\mathbb{R}_{+} \times \mathbb{S}}\left(\widehat{\chi}_{n} \otimes \widehat{\chi}_{n} * \widehat{\Psi}_{h}\right)(-k, \omega, k, \omega) 2 k d k d S^{2}(\omega)
$$

The Fourier transform of $F=F\left(\ell_{1}, \ell_{2}, \omega_{1}, \omega_{2}\right)$, above indicated by $\widehat{F}\left(k_{1}, k_{2}, \omega_{1}, \omega_{2}\right)$ has to be computed in $\mathbb{R}^{2}$ with respect to the variable $\left(\ell_{1}, \ell_{2}\right)$ and passing to the conjugate variable $\left(k_{1}, k_{2}\right) \in \mathbb{R}^{2}$. Finally it is restricted to the diagonal taking $k_{1}=k_{2}$ (and $\omega_{1}=\omega_{2}$ ). Furthermore, $*$ stands for the convolution in $\mathbb{R}^{2}$ so that, obviously, it results: $\widehat{\chi_{n} \otimes \chi_{n}} * \widehat{\Psi}_{h}=\widehat{\chi}_{n} \otimes \widehat{\chi}_{n} * \widehat{\Psi}_{h}=\chi_{n} \widehat{\otimes \chi_{n}} \Psi_{h}=\widehat{\chi_{n} \Psi_{h} \chi_{n}}$. To justify (59), we notice that it is simply proved that the right-hand side is weakly continuous as a function of $h \in C_{0}^{\infty}(M \times M ; \mathbb{R})$ (due to the continuity of $E \otimes E$ in the appropriate topologies and the presence of the cut-off functions $\chi_{n} \otimes \chi_{n}$ which restrict the image of $\Gamma E \otimes \Gamma E$ to a class of functions supported in a compact subset of $\left.\Im^{-} \times \Im^{-}\right)$. On the other hand, when $h=f \otimes g$ with $f, g \in C_{0}^{\infty}(M ; \mathbb{R})$, the right-hand side reduces to $\lambda_{n}(f, g)$ written as in the right-hand side of (42). Thus, by the uniqueness of the Schwartz kernel associated with a separately sequentially continuous quadratic form, the right-hand side of (59) individuates the distribution in $\mathcal{D}^{\prime}(M \times M)$ associated with the quadratic form $\lambda_{n}(\cdot, \cdot)$. Now define the 
functional over $C_{0}^{\infty}(M \times M ; \mathbb{R})$ :

$$
\lambda_{M}^{\prime}(h) \doteq \int_{\mathbb{R}_{+} \times \mathbb{S}} \widehat{\Psi}_{h}(-k, \omega, k, \omega) 2 k d k d S^{2}(\omega) .
$$

A priori, there is no guarantee that it defines an element of $\mathcal{D}^{\prime}(M \times M)$, i.e. that it is weakly continuous, nor that it is the distribution associated with the quadratic form $\lambda_{M}$. However, according to (36), it results $\lambda_{M}^{\prime}(f \otimes g)=\lambda_{M}(f, g)$. So, if $\lambda_{M}^{\prime}$ individuated an element of $\mathcal{D}^{\prime}(M \times M)$, it would have to coincide with the distribution associated with the quadratic form $\lambda_{M}(\cdot, \cdot)$, again in view of the uniqueness of the Schwartz kernel. Summarising, to prove that $\lambda_{M}^{\prime}=\lambda_{M} \in \mathcal{D}^{\prime}(M \times M)$ it is sufficient to prove that

$$
\lim _{n \rightarrow \infty}\left|\lambda_{n}(h)-\lambda_{M}^{\prime}(h)\right|=0 .
$$

In turn it would imply (58). To prove (61) we follow a procedure similar to that employed in the second part of the proof of the item (a) in Proposition 3.2. In other words we start considering a trivial partition of unit, constructed out of $\rho_{\delta}$ and $\rho_{\delta}^{\prime}$ as follows: we choose $\rho_{\delta} \in C_{0}^{\infty}(\mathbb{R})$ in such a way that $\rho_{\delta}(0)=1$, $0 \leq\left|\rho_{\delta}(k)\right| \leq 1$ and $\operatorname{supp}\left(\rho_{\delta}\right) \subset[-\delta, \delta]$, with $\delta>0$, whereas $\rho_{\delta}^{\prime} \doteq 1-\rho_{\delta}$. Therefore

$$
\widehat{\Psi}_{h}\left(k_{1}, \omega, k_{2}, \omega^{\prime}\right)=\left(\rho_{\delta}\left(k_{1}\right) \rho_{\delta}\left(k_{2}\right)+\rho_{\delta}\left(k_{1}\right) \rho_{\delta}^{\prime}\left(k_{2}\right)+\rho_{\delta}^{\prime}\left(k_{1}\right) \rho_{\delta}\left(k_{2}\right)+\rho_{\delta}^{\prime}\left(k_{1}\right) \rho_{\delta}^{\prime}\left(k_{2}\right)\right) \widehat{\Psi}_{h}\left(k_{1}, \omega, k_{2}, \omega^{\prime}\right),
$$

an expression we can now plug in $\lim _{n \rightarrow \infty}\left|\lambda_{n}(h)-\lambda_{M}^{\prime}(h)\right|$ to get four terms:

$$
\begin{gathered}
\int_{\mathbb{R}_{+} \times \mathbb{S}^{2}}\left|\widehat{\chi_{n} \widehat{\Psi}_{h} \chi_{n}}-\widehat{\Psi}_{h}\right| 2 k d k d S^{2} \leq \int_{\mathbb{R} \times \mathbb{S}^{2}}\left|\widehat{\chi_{n}} *\left(\rho_{\delta} \widehat{\Psi}_{h} \rho_{\delta}\right) * \widehat{\chi_{n}}-\rho_{\delta} \widehat{\Psi}_{h} \rho_{\delta}\right||2 k| d k d S^{2}+ \\
+\int_{\mathbb{R} \times \mathbb{S}^{2}}\left|\widehat{\chi_{n}} *\left(\rho_{\delta} \widehat{\Psi}_{h} \rho_{\delta}^{\prime}\right) * \widehat{\chi_{n}}-\rho_{\delta} \widehat{\Psi}_{h} \rho_{\delta}^{\prime}\right||2 k| d k d S^{2}+ \\
\int_{\mathbb{R} \times \mathbb{S}^{2}}\left|\widehat{\chi_{n}} *\left(\rho_{\delta}^{\prime} \widehat{\Psi}_{h} \rho_{\delta}\right) * \widehat{\chi_{n}}-\rho_{\delta}^{\prime} \widehat{\Psi}_{h} \rho_{\delta}\right||2 k| d k d S^{2}+\int_{\mathbb{R} \times \mathbb{S}^{2}}\left|\widehat{\chi_{n}} *\left(\rho_{\delta}^{\prime} \widehat{\Psi}_{h} \rho_{\delta}^{\prime}\right) * \widehat{\chi_{n}}-\rho_{\delta}^{\prime} \widehat{\Psi}_{h} \rho_{\delta}^{\prime}\right||2 k| d k d S^{2} .
\end{gathered}
$$

Henceforth, we shall indicate by $A_{n}, B_{n}, C_{n}$ and $D_{n}$, respectively, the four integrals in the right-hand side. Let us consider $D_{n}$. We have that, as $n \rightarrow+\infty$ :

$$
\begin{gathered}
\left(\widehat{\chi_{n}} *\left(\rho_{\delta}^{\prime} \widehat{\Psi}_{h} \rho_{\delta}^{\prime}\right) * \widehat{\chi_{n}}\right)\left(k, p, \omega, \omega^{\prime}\right)= \\
=\int_{\mathbb{R}^{2}} d k^{\prime} d p^{\prime}\left(\widehat{\chi}\left(k^{\prime}\right)\left(\rho_{\delta}^{\prime} \widehat{\Psi}_{h} \rho_{\delta}^{\prime}\right)\left(k-\frac{k^{\prime}}{n}, p-\frac{p^{\prime}}{n}, \omega, \omega^{\prime}\right) \widehat{\chi}\left(p^{\prime}\right)\right) \rightarrow\left(\rho_{\delta}^{\prime} \widehat{\Psi}_{h} \rho_{\delta}^{\prime}\right)\left(k, p, \omega, \omega^{\prime}\right) .
\end{gathered}
$$

Above we have used the dominated convergence since $\rho_{\delta}^{\prime} \widehat{\Psi}_{h} \rho_{\delta}^{\prime}=\rho_{\delta}^{\prime} \widehat{\Psi}_{h} \rho_{\delta}^{\prime}\left(k, p, \omega, \omega^{\prime}\right)$ is in the Schwartz space (the divergence has been cancelled by $\rho_{\delta}^{\prime}$ ) and $\widehat{\chi}$ is bounded. With the same argument used in the proof second part of the proof of the item (a) in Proposition 3.2 to achieve (44), one sees that there is a constant $K \geq 0$ with

$$
\left|\left(\widehat{\chi_{n}} *\left(\rho_{\delta}^{\prime} \widehat{\Psi}_{h} \rho_{\delta}^{\prime}\right) * \widehat{\chi_{n}}\right)\left(k, p, \omega, \omega^{\prime}\right)\right| \leq \frac{K}{\left(1+k^{2}\right)\left(1+p^{2}\right)} .
$$

So, it is possible to use again Lebesgue's theorem in $D_{n}$, obtaining $D_{n} \rightarrow 0$ for $n \rightarrow+\infty$.

Concerning $A_{n}$, we notice that it has to hold

$$
\left|A_{n}\right| \leq \int_{\mathbb{R} \times \mathbb{S}^{2}}\left|\widehat{\chi_{n}} *\left(\rho_{\delta} \widehat{\Psi}_{h} \rho_{\delta}\right) * \widehat{\chi_{n}}\right||2 k| d k d S^{2}+\int_{\mathbb{R} \times \mathbb{S}^{2}}\left|\rho_{\delta} \widehat{\Psi}_{h} \rho_{\delta}\right||2 k| d k d S^{2} .
$$


The last term is bounded by

$$
\int_{\mathbb{R} \times \mathbb{R}} d p d p^{\prime} \int_{\mathbb{R} \times \mathbb{S}^{2}} d k d S^{2}(\omega) 2|k|\left|\widehat{\chi_{n}}(-k-p)\left(\rho_{\delta} \widehat{\Psi}_{h} \rho_{\delta}\right)\left(p, p^{\prime}, \omega, \omega\right) \widehat{\chi_{n}}\left(k-p^{\prime}\right)\right|,
$$

Noticing that $\widehat{\chi}_{n}(p)=n \widehat{\chi}(n p)$, and passing from the coordinates $p, p^{\prime}, k$ to $u=n p, u^{\prime}=n p^{\prime}, h=n k$, the expression above can be bounded by:

$$
\frac{1}{n^{2}} \int_{\mathbb{R} \times \mathbb{R}} d u d u^{\prime} \int_{\mathbb{R} \times \mathbb{S}^{2}} d h d S^{2}(\omega) 2(|h+u|+|u|)\left|\widehat{\chi}(-h-u)\left(\rho_{\delta} \widehat{\Psi}_{h} \rho_{\delta}\right)\left(\frac{u}{n}, \frac{u}{n}, \omega, \omega\right) \widehat{\chi}\left(h-u^{\prime}\right)\right|,
$$

Using the fact that $|\widehat{\chi}|$ is bounded and has finite integral, the found integral can be bounded by:

$$
\frac{1}{n^{2}} \int_{[-n \delta,+n \delta] \times[-n \delta,+n \delta]} d u d u^{\prime} \int_{\mathbb{S}^{2}} d S^{2}(\omega)\left(K+K^{\prime}|u|\right)\left|\widehat{\Psi}_{h}\left(\frac{u}{n}, \frac{u}{n}, \omega, \omega\right)\right| .
$$

From the estimate (76), for small $k_{1}$ and $k_{2}$, we have $\widehat{\Psi}_{h}\left(k_{1}, k_{2}, \omega, \omega^{\prime}\right) \leq C /\left(\left|k_{1} k_{2}\right|^{R e \nu-1 / 2}\right)$. This bound, inserted in the integral above implies that, for some constants $K_{1}, K_{2} \geq 0$ independent from $\delta$ :

$$
\frac{1}{n^{2}} \int_{[-n \delta,+n \delta] \times[-n \delta,+n \delta]} d u d u^{\prime} \int_{\mathbb{S}^{2}} d S^{2}(\omega)\left(K+K^{\prime}|u|\right)\left|\widehat{\Psi}_{h}\left(\frac{u}{n}, \frac{u}{n}, \omega, \omega\right)\right| \leq K_{1} \delta^{3-2 R e \nu}+K_{2} \delta^{4-2 R e \nu},
$$

that is, looking back at (62):

$$
\int_{\mathbb{R} \times \mathbb{S}^{2}}\left|\widehat{\chi_{n}} *\left(\rho_{\delta} \widehat{\Psi}_{h} \rho_{\delta}\right) * \widehat{\chi_{n}}\right||2 k| d k d S^{2} \leq K_{1} \delta^{3-2 R e \nu}+K_{2} \delta^{4-2 R e \nu} .
$$

Analogously, we find:

$$
\int_{\mathbb{R} \times \mathbb{S}^{2}}\left|\left(\rho_{\delta} \widehat{\Psi}_{h} \rho_{\delta}\right)\right||2 k| d k d S^{2}(\omega) \leq \int_{[-\delta, \delta] \times \mathbb{S}^{2}}\left|\widehat{\Psi}_{h}\right||2 k| d k d S^{2}(\omega) \leq C^{\prime \prime} \delta^{3-2 R e \nu},
$$

where $C^{\prime}, C^{\prime \prime} \geq 0$ are constants independent on $\delta$. Therefore we have obtained that $\left|A_{n}\right| \leq H \delta^{3-2 R e \nu}$, uniformly in $n$, for some constant $H \geq 0$ independent from $\delta$. The remaining terms, $B_{n}$ and $C_{n}$, can be treated similarly making use of (76) with $n=2$, obtaining that, uniformly in $n,\left|B_{n}\right| \leq H^{\prime} \delta^{3-2 R e \nu}$ and $\left|C_{n}\right| \leq H^{\prime \prime} \delta^{3-2 R e \nu}$ for some constants $H^{\prime}, H^{\prime \prime}$ independent from $\delta$. Following the same procedure as in the last part of the proof of Proposition 3.2 (based on the standard properties of lim inf, lim sup), we can finally assert that the sequence of distributions $\lambda_{n}$ tends to $\lambda_{M}^{\prime}=\lambda_{M} \in \mathcal{D}^{\prime}(M \times M)$ weakly.

We are now in the position to study the convergence of the $\lambda_{n}$ to $\lambda_{M}$ in the Hörmander pseudotopology $\mathcal{D}_{\mathcal{V}}^{\prime}(M \times M)$. The following proposition holds, which easily implies the item (2) in Sec. 3.2.3 when employing a partition of the unit subordinated to a covering made of domains of coordinate patches on $M \times M$ and taking into account the compactness of the support of the $h$ appearing in the item (2) in Sec. 3.2.3.

Proposition 3.6. Let $U \subset M \times M$ a coordinate patch, $V \subset \mathbb{R}^{4} \times \mathbb{R}^{4}$ a close conic set, so that $U \times V$ can be viewed as the corresponding portion of $T^{*} M \times T^{*} M$ employing the coordinates over $U$, and let $h \in C_{0}^{\infty}(M \times M ; \mathbb{C})$ be a function supported in $U$. If $($ supph $\times V) \cap \mathcal{V}=\emptyset$, then:

$$
\sup _{n} \sup _{p \in V}|p|^{N}\left|\lambda_{n}\left(e^{i\langle p, \cdot\rangle} h\right)\right|<+\infty \quad \forall N=1,2, \ldots
$$


Proof. Obviously, by linearity, we can always assume which $h$ is real-valued and we shall assume it henceforth. It holds $\lambda_{n}\left(e^{i\langle p, \cdot\rangle} h\right)=\left|K\left(e^{i\langle p, \cdot\rangle} h \otimes \chi_{n} \otimes \chi_{n}\right)\right|$ in accordance with the definition of the distributions $\lambda_{n}$ in terms of the kernel $K$ as specified in (55). Thus, with $V$ as in the hypotheses, the following inequality holds for every $N=1,2, \ldots$, because $p \notin W F\left(\lambda_{n}\right)$ (since $W F\left(\lambda_{n}\right) \subset \mathcal{V}$ for (57)):

$$
\left|K\left(\chi_{n} \otimes \chi_{n} \otimes e^{i\langle p, \cdot\rangle} h\right)\right| \leq \frac{C_{N, n}}{(1+|p|)^{N}}, \quad \forall p \in V,
$$

for some constants $C_{N, n} \geq 0$ (depending on $h$ ). The idea is to show that, for any fixed $h \in C_{0}^{\infty}(M \times M ; \mathbb{R}$ ) there is $m_{h} \in \mathbb{N}$, such that one can take $C_{N, n}=C_{N, m_{h}}$ constantly, for $n \geq m_{h}$ in (64). This fact would lead to (63) immediately, since it implies that:

$$
\sup _{n} \sup _{p \in V}|p|^{N}\left|\lambda_{n}\left(e^{i\langle p, \cdot\rangle} h\right)\right| \leq \sup _{n} \sup _{p \in V} \frac{|p|^{N} C_{N, n}}{(1+|p|)^{N}} \leq \sup _{n} C_{N, n} \leq \max \left\{C_{N, 1}, C_{N, 2}, \ldots, C_{N, m_{h}}\right\}<+\infty .
$$

We need a preliminary lemma whose proof stays in the Appendix C.

Lemma 3.1. If $\mathcal{O} \subset \overline{\mathcal{O}} \subset M$ is any open, relatively compact set, there is $\ell_{\mathcal{O}}>0$ such that, viewing $\Gamma E$ a Schwartz kernel:

$$
\operatorname{sing} \operatorname{supp}\left(\Gamma E\left\lceil_{\Im^{-} \times \mathcal{O}}\right)\right) \subset \mathcal{N}_{\mathcal{O}} \times \mathcal{O}
$$

with $\mathcal{N}_{\mathcal{O}} \doteq\left(-\ell_{\mathcal{O}}, \ell_{\mathcal{O}}\right) \times \mathbb{S}^{2}$ in $\Im^{-}$.

To go on we give a precise definition of the functions $\chi_{n}$. As usual $\chi_{n}(\ell, \omega) \doteq \chi(\ell / n)$, but now we define $\chi \in C_{0}^{\infty}\left(\mathbb{R} \times \mathbb{S}^{2} ; \mathbb{R}\right)$ as a function independent form $\omega \in \mathbb{S}^{2}$, with $0 \leq \chi(\ell) \leq 1$ and $\chi(\ell)=0$ for $|\ell| \geq 2$ whereas $\chi(\ell)=1$ for $|\ell| \leq 1$. Notice that the support of $\chi_{n}$ becomes larger and larger as $n$ increases and tends to cover the whole $\Im^{-}$, taking everywhere the value 1 , as $n \rightarrow+\infty$. Since $M$ is homeomorphic to $\mathbb{R}^{4} \times \mathbb{R}^{4}$, for every $h \in C_{0}^{\infty}(M \times M)$, there is a set $\mathcal{O} \doteq \mathcal{O}_{h}$, as in the hypotheses of the Lemma 3.1, such that $\mathcal{O}_{h} \times \mathcal{O}_{h} \supset$ supp $h$. Hence, there exists a sufficiently large $n_{h} \in \mathbb{N}$ such that $\mathcal{N}_{\mathcal{O}_{h}} \subset$ supp $\chi_{n}$ as well as $\chi_{n}\left(\overline{\mathcal{N}_{\mathcal{O}_{h}}}\right)=1$ if $n \geq n_{h}$.

We have the following bound for $K\left(e^{i\langle p, \cdot\rangle} h \otimes \chi_{n} \otimes \chi_{n}\right)$ :

$$
\begin{gathered}
\left|K\left(\chi_{n} \otimes \chi_{n} \otimes e^{i\langle p, \cdot\rangle} h\right)\right| \leq \\
\left|K\left(\chi_{n_{h}} \otimes \chi_{n_{h}} \otimes e^{i\langle p, \cdot\rangle} h\right)\right|+\left|K\left(\left(\chi_{n}-\chi_{n_{h}}\right) \otimes \chi_{n_{h}} \otimes e^{i\langle p, \cdot\rangle} h\right)\right|+ \\
\left|K\left(\chi_{h_{n}} \otimes\left(\chi_{n}-\chi_{n_{h}}\right) \otimes e^{i\langle p, \cdot\rangle} h\right)\right|+\left|K\left(\left(\chi_{n}-\chi_{n_{h}}\right) \otimes\left(\chi_{n}-\chi_{n_{h}}\right) \otimes e^{i\langle p, \cdot\rangle} h\right)\right|
\end{gathered}
$$

Let us indicate by $P_{n}, Q_{n}$ and $R_{n}$ the first the third and the last term, respectively, in the right-hand side of the inequality above. The second can be discussed similarly to the third. Let us analyze the features of $P_{n}, Q_{n}$ and $R_{n}$ separately proving that there exist a natural $m_{h}$ which guarantees the validity of the thesis as discussed above, for each term separately, thus concluding the proof.

Analysis of $P_{n}$. The analysis is straightforward because $n=n_{h}$ is fixed. As $p \in V$ does not belong to the $W F\left(\lambda_{n_{h}}\right)$, for every $N$ it exist $C_{N, n_{h}} \geq 0$ such that

$$
P_{n}=\left|K\left(\chi_{n_{h}} \otimes \chi_{n_{h}} \otimes e^{i\langle p \cdot\rangle} h\right)\right| \leq \frac{C_{N, n_{h}}}{(1+|p|)^{N}}, \quad \forall p \in V, \quad \forall N \geq 1 .
$$

Analysis of $R_{n}$. With our definition of $n_{h}$, the function $\chi_{n}-\chi_{n_{h}}$ vanishes over $\overline{\mathcal{N}_{\mathcal{O}}}$ for $n \geq n_{h}$. Thus, due to the Lemma 3.1, the wave front set of $\left(\left(\chi_{n}-\chi_{n_{h}}\right) \Gamma E \otimes\left(\chi_{n}-\chi_{n_{h}}\right) \Gamma E\right) \Upsilon_{\Im^{-} \times \Im^{-} \times \mathcal{O} \times \mathcal{O}}$ is 
empty, so that every $p$ in $\mathbb{R}^{4} \times \mathbb{R}^{4}$ individuates a direction of rapid decrease for it. As supph $\subset \mathcal{O}_{h}$, this result allows us to estimate the rate of rapid decreasing of $K\left(\left(\chi_{n}-\chi_{n_{h}}\right) \otimes\left(\chi_{n}-\chi_{n_{h}}\right) \otimes e^{i\langle p, \cdot\rangle} h\right)$. In the Appendix $\mathrm{C}$, we shall prove the following lemma

Lemma 3.2. With the hypotheses of Proposition 3.6, for every $N=1,2, \ldots$, there is a constant $C_{N} \geq 0$ such that:

$$
\left|k_{1}\right|^{2}\left|k_{2}\right|^{2}|p|^{N}\left|(\Gamma E \otimes \Gamma E)\left(\left(\chi_{n}-\chi_{n_{h}}\right) e^{i\left\langle k_{1}, \cdot\right\rangle} \otimes\left(\chi_{n}-\chi_{n_{h}}\right) e^{i\left\langle k_{2}, \cdot\right\rangle} \otimes h e^{i\langle p, \cdot\rangle}\right)\right| \leq C_{N},
$$

when $n \geq n_{h}$.

Let us prove that (66) implies that, if $n \geq n_{h}$ :

$$
R_{n}=\left|K\left(\left(\chi_{n}-\chi_{n_{h}}\right) \otimes\left(\chi_{n}-\chi_{n_{h}}\right) \otimes e^{i\langle p, \cdot\rangle}\right)\right| \leq \frac{C_{N}}{(1+|p|)^{N}}, \quad \forall p \in V, \forall N \geq 1,
$$

for some $C_{N} \geq 0$. Indeed, (66) entails that, for every $N$, it exist a $C_{N}^{\prime} \geq 0$, which does not depend on $n \geq n_{h}$, such that:

$$
\left|(\Gamma E \otimes \Gamma E)\left(\left(\chi_{n}-\chi_{n_{h}}\right) e^{i\left\langle k_{1}, \cdot\right\rangle} \otimes\left(\chi_{n}-\chi_{n_{h}}\right) e^{i\left\langle k_{2}, \cdot\right\rangle} \otimes h e^{i\langle p, \cdot\rangle}\right)\right| \leq \frac{1}{\left(1+\left|k_{1}\right|\right)^{2}\left(1+\left|k_{2}\right|\right)^{2}} \frac{C_{N}^{\prime}}{(1+|p|)^{N}} .
$$

The left-hand side is nothing but $H^{2}\left|\left(\left(\widehat{\chi_{n}-\chi_{n_{h}}}\right) \otimes\left(\chi_{n}-\chi_{n_{h}}\right) * \widehat{\Psi}_{h e^{i\langle p, \cdot\rangle}}\right)\left(k_{1}, \omega, k_{2}, \omega^{\prime}\right)\right|$. Therefore, from the very definition of the kernel $K, K\left(\left(\chi_{n}-\chi_{n_{h}}\right) \otimes\left(\chi_{n}-\chi_{n_{h}}\right) \otimes e^{i\langle p, \cdot\rangle}\right)$ is obtained by integrating $(\Gamma E \otimes \Gamma E)\left(\left(\chi_{n}-\chi_{n_{h}}\right) e^{i\left\langle k_{1}, \cdot\right\rangle} \otimes\left(\chi_{n}-\chi_{n_{h}}\right) e^{i\left\langle k_{2}, \cdot\right\rangle} \otimes h e^{i\langle p, \cdot\rangle}\right)$ with $k_{1}=-k_{2}=k$ and $\omega=\omega^{\prime}$ over $\mathbb{R}_{+} \times \mathbb{S}^{2}$ with respect to the measure $k d k d S^{2}(\omega)$. In that way, (68) yields that $C_{N} \geq 0$ exists such that, $\forall n \geq n_{h}$ :

$$
\left|K\left(\left(\chi_{n}-\chi_{n_{h}}\right) \otimes\left(\chi_{n}-\chi_{n_{h}}\right) \otimes e^{i\langle p, \cdot\rangle}\right)\right| \leq 4 \pi\left(\int_{0}^{+\infty} \frac{2 k d k}{(1+|k|)^{4}}\right) \frac{C_{N}^{\prime}}{(1+|p|)^{N}}=\frac{C_{N}}{(1+|p|)^{N}}, \quad \forall p \in V, \forall N \geq 1 .
$$

We have finally proved the validity of (67).

Analysis of $Q_{n}$. We start by:

$\left.Q_{n}=\mid K\left(\chi_{n_{h}} \otimes\left(\chi_{n}-\chi_{n_{h}}\right) \otimes e^{i\langle p, \cdot\rangle} h\right)\right)|\leq| K\left(\chi_{n_{h}} \otimes\left(\chi_{n}-\chi_{3 n_{h}}\right) \otimes e^{i\langle p, \cdot\rangle} h\right)|+| K\left(\chi_{n_{h}} \otimes\left(\chi_{3 n_{h}}-\chi_{n_{h}}\right) \otimes e^{i\langle p, \cdot\rangle} h\right) \mid$.

First of all notice that the wave front set of $W F\left(K\left(\chi_{n_{h}} \otimes\left(\chi_{3 n_{h}}-\chi_{n_{h}}\right) \otimes \cdot\right)\right) \subset \mathcal{V}$, as $K\left(\chi_{n_{h}} \otimes\left(\chi_{3 n_{h}}-\chi_{n_{h}}\right) \otimes \cdot\right)$ can be seen as a composition of distribution with compact support and the Theorem 8.2.14 of [Hö89] can be applied twice. Therefore, every $p \in V$ is a direction of rapid decreasing for such distribution and the rate of decrease does not depend on $n$ by construction. Let us pass to analyse the first term in the right-hand side of the inequality written above for $Q_{n}$. Notice that the support of $\chi_{n_{h}}$ never intersects the support of $\left(\chi_{n}-\chi_{3 n_{h}}\right)$ if $n \geq 3 n_{h}$, hence the singularity $\left(\ell-\ell^{\prime}\right)^{-2}$ present inside $K$ due to $T$ (see (46) and Proposition 3.3) is harmless. For this reason we can skip the $\epsilon$-prescription present in $T$ (reminded by $0^{+}$in (46)) and we can consider the part of the integral kernel of $T$ depending on $\ell$ and $\ell^{\prime}$ as a smooth function. We pass to establish the existence of a $n$-uniform bound for $|p|^{N}\left|K\left(\chi_{n_{h}} \otimes\left(\chi_{n}-\chi_{3 n_{h}}\right) \otimes e^{i\langle p, \cdot\rangle} h\right)\right|$. We have the bound, where, as before, $y=\left(y_{1}, y_{2}\right)$ and with obvious notation concerning derivatives:

$$
\begin{gathered}
|p|^{N}\left|K\left(\chi_{n_{h}} \otimes\left(\chi_{n}-\chi_{3 n_{h}}\right) \otimes e^{i\langle p, \cdot\rangle} h\right)\right| \leq \\
\leq\left|\int d \ell d S^{2}(\omega) d \mu\left(y_{1}\right) \Gamma E\left(\ell, \omega, y_{1}\right) \int d \mu\left(y_{2}\right) e^{i\langle p, y\rangle} D_{y}^{N} h(y) \chi_{n_{h}}(\ell) \int \mathcal{E}\left(\ell^{\prime}, \omega, y_{2}\right) \frac{\chi\left(\frac{\ell^{\prime}}{n}\right)-\chi_{3 n_{h}}\left(\ell^{\prime}\right)}{\left(\ell-\ell^{\prime}\right)^{2}} d \ell^{\prime}\right| .
\end{gathered}
$$


Notice that due to the domain property of $h$ and $\chi\left(\ell^{\prime} / n\right)-\chi_{3 n_{h}}\left(\ell^{\prime}\right)$, the associated causal propagator is a smooth function similarly to the discussion done for the term $R_{n}$ mande in the proof of Lemma 3.2. As we done in the proof of that lemma, we have denoted it by $\mathcal{E}\left(\ell^{\prime}, \omega^{\prime}, y_{2}\right)$ in the formula above, where the two internal integrations have the standard meaning, whereas the external one has to be understood in the distributional sense. To find an estimate for the right-hand side of (69) it is convenient to define, for $n=3 n_{h}, 3 n_{h}+1, \ldots, \infty$ :

$$
F_{n}^{\beta}(\ell, \omega, y) \doteq \rho(y) \int_{\mathbb{R}} D_{y_{2}}^{\beta} \mathcal{\varepsilon}\left(\ell^{\prime}, \omega, y_{2}\right) \chi_{n_{h}}(\ell) \frac{\left[\chi\left(\frac{\ell^{\prime}}{n}\right)-\chi_{3 n_{h}}\left(\ell^{\prime}\right)\right]}{\left(\ell-\ell^{\prime}\right)^{2}} d \ell^{\prime}
$$

and

$$
F_{\infty}^{\beta}(\ell, \omega, y) \doteq \rho(y) \int_{\mathbb{R}} D_{y_{2}}^{\beta} \mathcal{E}\left(\ell^{\prime}, \omega, y_{2}\right) \chi_{n_{h}}(\ell) \frac{1-\chi_{3 n_{h}}\left(\ell^{\prime}\right)}{\left(\ell-\ell^{\prime}\right)^{2}} d \ell^{\prime}, \quad G_{n}^{\beta}(y) \doteq \rho(y)^{t}(\Gamma E)\left(F_{n}^{\beta}\right)(y)
$$

where $\rho(y)=\rho_{1}\left(y_{1}\right) \rho_{2}\left(y_{2}\right)$ is such that $\rho_{1}, \rho_{2} \in C_{0}^{\infty}(M ; \mathbb{R})$ and $\rho(y)=1$ if $y \in \overline{\mathcal{O}_{h} \times \mathcal{O}_{h}}$. Notice that, because of the decay property at large $\ell^{\prime}$ of $\mathcal{E}$ discussed in the analysis of $R_{n}$, and due of the boundedness of $\chi(\cdot / n)-\chi_{3 n_{h}}(\cdot)$, all the $F_{n}^{\beta}$ as well as $F_{\infty}^{\beta}$ are smooth functions. So they belong to $C_{0}^{\infty}\left(\Im^{-} \times M \times M ; \mathbb{R}\right)$ by construction. By direct inspection one proves that $F_{n}^{\beta} \rightarrow F_{\infty}^{\beta}$ in the topology of $C_{0}^{\infty}\left(\Im \Im^{-} \times M \times M ; \mathbb{C}\right)$, as $n \rightarrow+\infty$. Furthermore, in view of Theorem 8.2.12 and the discussion before Theorem 8.2.13 in [Hö89] (and we adopt here the notation used therein), as $W F\left({ }^{t}(\Gamma E)\right)_{M}$ is the empty set, it results that $G_{n}$ is smooth on $M \times M$ for every $n \geq 3 n_{h}$ including $n=\infty$, it is compactly supported within a $n$-independent relatively compact set including $\overline{\mathcal{O}_{h}}$, in view of the presence of $\rho$, and $G_{n} \rightarrow G_{\infty}$ in the topology of $C_{0}^{\infty}(M \times M)$ as $3 n_{h} \leq n \rightarrow+\infty$. Equipped with the introduced functions, the right hand side of (69) can be rearranged as

$$
\left|\int e^{i\langle p, y\rangle} D_{y}^{N} G_{n}^{0}(y) h(y) d \mu\left(y_{1}\right) d \mu\left(y_{2}\right)\right|
$$

so that we finally have:

$$
|p|^{N}\left|K\left(\chi_{n_{h}} \otimes\left(\chi_{n}-\chi_{3 n_{h}}\right) \otimes e^{i\langle p, \cdot\rangle} h\right)\right| \leq \sum_{\beta+\beta_{1}+\beta_{2}=N} \int\left|D_{y_{2}}^{\beta_{2}}\left(G_{n}^{\beta}(y) D_{y_{1}}^{\beta_{1}} h(y)\right)\right| d \mu\left(y_{1}\right) d \mu\left(y_{2}\right) \leq C_{N, n}^{\prime}
$$

However, since $G_{n} \rightarrow G_{\infty}$ in the topology of $C_{0}^{\infty}(M \times M)$ as $3 n_{h} \leq n \rightarrow+\infty$, there exists $C_{N}<+\infty$ with $C_{N, n}^{\prime} \leq C_{N}$, so that, for $n \geq 3 n_{h}$ :

$$
Q_{n}=\left|K\left(\chi_{n_{h}} \otimes\left(\chi_{n}-\chi_{n_{h}}\right) \otimes e^{i\langle p, \cdot\rangle} h\right)\right| \leq \frac{C_{N}}{(1+|p|)^{N}}, \quad \forall p \in V, \quad \forall N \geq 1
$$

Finally, collecting the estimates for the four terms in (65) we get that (64) holds true with $C_{N, n}=C_{N, m_{h}}$ constantly if $n \geq m_{h}$ whenever one assumes $m_{h} \doteq 3 n_{h}$, and this conclude the proof. $\square$.

As discussed in Sec. 3.2.3, the achieved result implies the inclusion $\subset$ in (35) and this concludes the proof of Theorem 3.1 .

\section{Conclusion}

In [DMP06] we established that, out of a bulk-to-boundary reconstruction procedure, it is possible to identify a preferred quasifree algebraic state for a scalar field theory living on any manifold lying in a 
large class of Friedmann-Robertson-Walker spacetimes. The first goal accomplished in the present paper is the extension of those results to other physically relevant cases, encompassing the analyses of the linear scalar fluctuations of the metric in inflationary models. As a further result, in this paper, we proved that such state is also of Hadamard form. This entails several interesting consequences the most notable being both the boundedness of the back-reaction due to quantum effects and the possibility to perform over such a state a renormalisation procedure. Despite the interest of these remarks, we should emphasise once more that the possibly most interesting application of our results lies in inflationary models according to which the early Universe undergoes an almost de Sitter phase of expansion driven by a real scalar field coupled to a self-interaction potential. Within this framework many consequences are derived employing quantisation and perturbative techniques and the most notable is the existence of a power spectrum of fluctuations which is almost scale invariant. A byproduct of this paper is indeed the possibility to put on a firmer mathematical ground the often taken for granted assertion that it exists a well-behaved quantum state out of which the above results can be derived.

Nonetheless we should point out that our proof holds true only under same further hypothesis and most notably $\nu$, taken as in (11), must differ from $\frac{3}{2}$ which would correspond to a massless scalar field minimally coupled to scalar curvature. In this scenario the techniques employed in this paper cannot be applied and we reckon that a rethinking of the whole procedure is necessary. Nonetheless we feel safe to claim that the proof of the Hadamard condition, as it stands now, is of physical interest from the point of view of cosmology and it opens the road to tackle specific inflationary models on a firm mathematical ground.

\section{Acknowledgements.}

The work of C. D. was supported by the von Humboldt Foundation and, that of N. P., by the German DFG Research Program SFB 676. Part of this paper was written by V. M. and N. P. at the E. Schrödinger Institute of Vienna during the ESI Program Operator Algebras and Conformal Field Theory. V. M. and N. P. are therefore grateful to the institute and the organisers of the program Y. Kawahigashi, R. Longo, K.-H. Rehren and J. Yngvason for their kind hospitality. The authors would like to thank K. Fredenhagen and R. Brunetti for useful discussions.

\section{A Approximated solution by means of perturbation theory}

We shall briefly comment both on the extension of the construction of the solution $\chi_{k}(\tau)$ of (9) in the case $\nu \in \mathbb{R}$ with $1 / 2<\nu<3 / 2$ and $V(\tau)=O\left(\tau^{-5}\right)$ as well as on the related uniform estimates that we have used throughout. The values $0 \leq \nu \leq 1 / 2$ (or $\nu$ imaginary) were considered in [DMP08]. The construction is similar to the one presented in the proof of theorem 4.5 in [DMP08] though some subtleties arise. To be more specific, in the mentioned theorem, $\chi_{k}$ was constructed by means of a perturbative series around a particular solution $\chi_{k}^{0}(\tau)$ which corresponds to (12), that in the de Sitter background. More precisely

$$
\begin{gathered}
\chi_{k}(\tau)=\chi_{k}^{0}(\tau) \\
+\sum_{n=1}^{+\infty}(-1)^{n} \int_{-\infty}^{\tau} d t_{1} \int_{-\infty}^{t_{1}} d t_{2} \cdots \int_{-\infty}^{t_{n-1}} d t_{n} S_{k}\left(\tau, t_{1}\right) S_{k}\left(t_{1}, t_{2}\right) \cdots S_{k}\left(t_{n-1}, t_{n}\right) V\left(t_{1}\right) V\left(t_{2}\right) \cdots V\left(t_{n}\right) \chi_{k}^{0}\left(t_{n}\right),
\end{gathered}
$$


where

$$
S_{k}\left(t, t^{\prime}\right):=-i\left(\overline{\chi_{k}^{0}(t)} \chi_{k}^{0}\left(t^{\prime}\right)-\overline{\chi_{k}^{0}\left(t^{\prime}\right)} \chi_{k}^{0}(t)\right), \quad t, t^{\prime} \in(-\infty, 0),
$$

is the retarded fundamental solution of the unperturbed one dimensional problem (9), whereas $V(\tau)$ is the perturbation potential. In the case under investigation, the proof of the convergence of the series (70) can be proved, dividing the problem into two parts, namely we shall discuss the cases $0 \leq k<1$ and $k \geq 1$ separately. Though the former is the most difficult, we can nonetheless take into account the following behaviour for $|k \tau| \leq 1$ :

$$
\left|\chi_{k}^{0}(\tau)\right| \leq \frac{C_{\nu}}{2} \sqrt{\tau}\left(|k \tau|^{\nu}+|k \tau|^{2-\nu}+\frac{1}{|k \tau|^{\nu}}\right), \quad|\tau|>1,|k \tau|<1
$$

as well as that for $|k \tau| \geq 1$

$$
\left|\chi_{k}^{0}(\tau)\right| \leq \frac{C_{\nu}}{\sqrt{|k|}}, \quad|\tau|>1,|k \tau| \geq 1 .
$$

Together they imply the following $\tau$-uniform estimate

$$
\left|\chi_{k}^{0}(\tau)\right| \leq \frac{C_{\nu}}{2}\left(\frac{1}{|k|^{\nu}}+\frac{1}{\sqrt{|k|}}\right), \quad|\tau|>1
$$

where $C_{\nu}$ is some positive $\nu$-dependant constant. The analysis of $S_{k}\left(t_{1}, t_{2}\right)$ is more subtle, we shall derive two estimates valid for small and large $k$ respectively. More precisely

$$
\left|S_{k}\left(t_{1}, t_{2}\right)\right| \leq C_{\nu}^{\prime}\left(\sqrt{\left|t_{1} t_{2}\right|}\right)\left(\left|t_{1}\right|^{\nu}\left|t_{2}\right|^{2-\nu}+\left|t_{1}\right|^{\nu}\left|t_{2}\right|^{2-\nu}\right), \leq C_{\nu}^{\prime}\left|t_{1}\right|^{2 \alpha+1},\left|t_{1}\right|>\left|t_{2}\right|>1,|k|<1
$$

where $0<\alpha \doteq \sup \{\nu, 2-\nu\}<\frac{3}{2}$, while

$$
\left|S_{k}\left(t_{1}, t_{2}\right)\right| \leq C_{\nu}^{\prime} \frac{1}{|k|}, \quad\left|t_{1}\right|>\left|t_{2}\right|>1, \quad|k| \geq 1
$$

$C_{\nu}^{\prime}$ being a further constant different from $C_{\nu}$. Thanks to these two estimates and since the perturbation potential is $V(\tau)=O\left(\tau^{-5}\right)$, the series (70) is dominated by the following convergent series

$$
\left|\chi_{k}(\tau)\right| \leq\left|\chi_{k}^{0}(\tau)\right| \sum_{n=0}^{\infty} \frac{1}{n !}\left(\frac{C}{(3-2 \alpha)|\tau|^{3-2 \alpha}}\right)^{n},|k|<1 \quad\left|\chi_{k}(\tau)\right| \leq\left|\chi_{k}^{0}(\tau)\right| \sum_{n=0}^{\infty} \frac{1}{n !}\left(\frac{C}{4|k||\tau|^{4}}\right)^{n},|k| \geq 1 .
$$

with a fixed constant $C$. The two inequalities show that the behaviour in $k$ of $\left|\chi_{k}(\tau)\right|$ is similar to the one presented in (72) for $\left|\chi_{k}^{0}(\tau)\right|$. It is possible to prove that the $\tau$ derivatives do not alter the bound given at small $k$. Notice that similar estimates hold true also for $\partial_{\tau} \chi_{k}(\tau)$. More precisely, since for $|k|<1$, both $S_{k}\left(t_{1}, t_{2}\right)$ and $\chi_{0}(\tau)$ can be expanded in powers of $k$ the following bound must hold for the $n$th order $\tau$ derivative of $\chi_{k}(\tau)$

$$
\partial_{\tau}^{n} \chi_{k}(\tau)=\frac{C_{\nu}^{n+1}}{2} \frac{1}{|k|^{\nu}}+O\left(|k|^{\min (\nu, 2-\nu)}\right), \quad|\tau|>1,|k|<1 .
$$

These estimates are valid for $\nu \in \mathbb{R}$ with $0 \leq \nu<3 / 2$. For $\nu \in i \mathbb{R}$ there is no singularity at $k=0$. To conclude this appendix we would like to emphasise that, as $\tau \rightarrow-\infty$, the analogy between $\chi_{k}^{0}$ and $\chi_{k}$ becomes stronger; most notably, if we rearrange the series (70) and we operate as before, in the limit $\tau \rightarrow \infty$, we end up with

$$
\left|\chi_{k}(\tau)-\chi_{k}^{0}(\tau)\right| \leq C_{\nu}\left(\frac{1}{|k|^{\nu}}+\frac{1}{\sqrt{|k|}}\right)\left(e^{C / \tau^{3-2 \alpha}}-1\right) \rightarrow 0, \quad \tau \rightarrow-\infty .
$$




\section{B Derivation of some estimates both for $\Phi_{h}$ and $\Psi_{h}$}

In this appendix we derive some estimates both for the bi-solutions of Klein-Gordon equation generated by real smooth compactly-supported smooth on $M \times M$ and for their restrictions to the horizon $\Im^{-} \times \Im^{-}$. To this end, take $h \in C_{0}^{\infty}(M \times M ; \mathbb{R})$ and define the two-wavefunction $\Phi_{h}$ and its extension/restriction $\Psi_{h}$ to $\Im^{-} \times \Im^{-}$(referring to the larger globally-hyperbolic spacetime $\left(\widehat{M}, \widehat{g}_{F R W}\right)$ including $\left(M, g_{F R W}\right)$ as a subspace):

$$
\Phi_{h} \doteq(E \otimes E) h, \quad \Psi_{h} \doteq H^{-2}[(\widehat{E} \otimes \widehat{E}) h] \Upsilon_{\Im^{-} \times \Im^{-}} .
$$

It result $\Phi_{h} \in C^{\infty}(M \times M ; \mathbb{R})$ and similarly $\Psi_{h} \in C^{\infty}\left(\Im^{-} \times \Im^{-} ; \mathbb{R}\right)$. It follows by direct application of Theorems 8.2.9 and 8.2.12 in [Hö89] and taking into account the shape of $W F(E)$ (49). Moreover, it turns out that the restriction of $\Phi_{h}$ to $\Sigma_{\tau} \times \Sigma_{\tau}$ has compact support when $\Sigma_{\tau}$ is a constant-time Cauchy surface of $\left(M, g_{F R W}\right)$. To prove it, take an open set $A \subset M$ such that $\bar{A}$ is compact and supph $\subset \bar{A} \times \bar{A}$ (such $A$ does exist because $M$ is homeomorphic to $\left.\mathbb{R}^{4}\right)$. Thus consider class of functions $f_{n}, g_{n} \in C_{0}^{\infty}(A ; \mathbb{R})$ with $\sum_{n=1}^{N} f_{n} \otimes g_{n} \rightarrow h$ in $C_{0}^{\infty}(A \times A ; \mathbb{C})$ as $N \rightarrow+\infty$. By the known properties of $E$ in globally hyperbolic spacetime, the restriction of $E f$ to a spacelike Cauchy surface $\Sigma$ is included in the compact $J(H) \cap \Sigma$ when $f \in C^{\infty}(M ; \mathbb{R})$ is such that supp $\subset \subset H$ and $H$ is compact. (Here and henceforth $J(A) \doteq J^{+}(A) \cup J^{-}(A)$.) As a consequence, the restriction of $(E \otimes E)\left(\sum_{n=1}^{N} f_{n} \otimes g_{n}\right)=\sum_{n=1}^{N} E\left(f_{n}\right) \otimes E\left(g_{n}\right)$ to $\Sigma_{\tau} \times \Sigma_{\tau}$ has support included in the compact $B \doteq\left(J(\bar{A}) \cap \Sigma_{\tau}\right) \times\left(J(\bar{A}) \cap \Sigma_{\tau}\right)$. Since $\sum_{n=1}^{N} E\left(f_{n}\right) \otimes E\left(g_{n}\right) \rightarrow h$ uniformly on every compact set, as $N \rightarrow+\infty$, and each $\left(E\left(f_{n}\right) \otimes E\left(g_{n}\right)\right)\left\lceil\Sigma_{\tau} \times \Sigma_{\tau}\right.$ vanishes outside $B$, we have that for every compact subset $B^{\prime}$ of $\Sigma_{\tau} \times \Sigma_{\tau}$ with $B^{\prime} \supset B$, it also holds $h \uparrow_{B^{\prime} \backslash B}=0$. Taking $B^{\prime}$ larger and larger (this is possible because $\Sigma_{\tau}$ is homeomorphic to $\mathbb{R}^{3}$ ) one finds that $\Phi_{h}\left\lceil_{\Sigma_{\tau} \times \Sigma_{\tau}}=0\right.$ outside the compact $B$, so that $\operatorname{supp}\left(\Phi_{h}\left\lceil\Sigma_{\tau} \times \Sigma_{\tau}\right)=\operatorname{supp}\left[((E \otimes E) h)\left\lceil\Sigma_{\tau} \times \Sigma_{\tau}\right] \subset B\right.\right.$ is compact.

$\Phi_{h}$ can be decomposed into modes along the lines of (8), formally:

$$
\Phi_{h}\left(x_{1}, x_{2}\right)=\int_{\mathbb{R}^{3} \times \mathbb{R}^{3}}\left[\phi_{\mathbf{k}_{1}}\left(x_{1}\right) \phi_{\mathbf{k}_{\mathbf{2}}}\left(x_{2}\right) \widetilde{\Phi}_{h}\left(\mathbf{k}_{1}, \mathbf{k}_{2}\right)+\overline{\phi_{\mathbf{k}_{\mathbf{1}}}\left(x_{1}\right) \phi_{\mathbf{k}_{\mathbf{2}}}\left(x_{2}\right) \widetilde{\Phi}_{h}\left(\mathbf{k}_{1}, \mathbf{k}_{2}\right)}\right] d^{3} \mathbf{k}_{1} d^{3} \mathbf{k}_{2} .
$$

Let us analyse the properties of $\widetilde{\Phi}_{h}\left(\mathbf{k}_{1}, \mathbf{k}_{2}\right)$ and its dependence on $h$. To this end, fix any, but fixed, initial time $\tau_{0}$ and the associated Cauchy surface $\Sigma_{\tau_{0}}$. Since $\Phi_{h}$ is smooth by construction, we can restrict it on $\Sigma_{\tau_{0}} \times \Sigma_{\tau_{0}}$. Hence the previous formula is invertible by means of a procedure similar to that of (15):

$$
\begin{aligned}
& \widetilde{\Phi}\left(\mathbf{k}_{1}, \mathbf{k}_{2}\right)=-i \int_{\Sigma_{\tau_{0}} \times \Sigma_{\tau_{0}}} d^{3} x_{1} d^{3} x_{2} a^{4}\left(\tau_{0}\right)\left[\frac{\partial \overline{\phi_{\mathbf{k}_{1}}\left(x_{1}\right)}}{\partial \tau_{1}} \frac{\partial \overline{\phi_{\mathbf{k}_{2}}\left(x_{2}\right)}}{\partial \tau_{2}} \Phi_{h}\left(x_{1}, x_{2}\right)+\right. \\
& \left.-\frac{\partial \overline{\phi_{\mathbf{k}_{2}}\left(x_{2}\right)}}{\partial \tau_{2}} \overline{\phi_{\mathbf{k}_{1}}\left(x_{1}\right)} \frac{\partial \Phi_{h}\left(x_{1}, x_{2}\right)}{\partial \tau_{1}} \overline{\phi_{\mathbf{k}_{1}}\left(x_{1}\right) \phi_{\mathbf{k}_{2}}\left(x_{2}\right)} \frac{\partial^{2} \Phi_{h}\left(x_{1}, x_{2}\right)}{\partial \tau_{1} \partial \tau_{2}}-\frac{\partial \overline{\phi_{\mathbf{k}_{1}}\left(x_{1}\right)}}{\partial \tau_{1}} \overline{\phi_{\mathbf{k}_{2}}\left(x_{2}\right)} \frac{\partial \Phi_{h}\left(x_{1}, x_{2}\right)}{\partial \tau_{2}}\right] \text {. }
\end{aligned}
$$

In combination both with the explicit form of the modes $\phi_{\mathbf{k}}$ as in (8) and with $\Phi_{h}, \partial_{\tau_{1}} \Phi_{h}, \partial_{\tau_{2}} \Phi_{h} \in$ $C_{0}^{\infty}\left(\Sigma_{\tau_{0}} \times \Sigma_{\tau_{0}} ; \mathbb{C}\right)$, such expression entails that $\widetilde{\Phi}_{h}\left(\mathbf{k}_{1}, \mathbf{k}_{2}\right)$ is an integrable function which is smooth except for $\mathbf{k}_{1}=0$ and $\mathbf{k}_{2}=0$ separately. Furthermore it decays rapidly at large $\mathbf{k}_{1}$ or $\mathbf{k}_{2}$ uniformly in the angles (when the other variable is fixed) whereas, near $\mathbf{k}_{1}=0$ and $\mathbf{k}_{2}=0$ it has the following angle-independent uniform bound:

$$
\left|\widetilde{\Phi}_{h}\left(\mathbf{k}_{1}, \mathbf{k}_{2}\right)\right| \leq \frac{C}{\left(\left|\mathbf{k}_{1}\right|\left|\mathbf{k}_{2}\right|\right)^{R e \nu}}
$$


The behaviour can be summarised as it follows $\widetilde{\Phi}_{h}=\widetilde{\Phi}_{h}\left(\mathbf{k}_{1}, \mathbf{k}_{2}\right)$ is everywhere smooth but $\mathbf{k}_{1}=0$ and $\mathbf{k}_{2}=0$ separately, moreover, for $n=1,2, \ldots$ there are constants $C_{n} \geq 0$ with:

$$
\left|\widetilde{\Phi}_{h}\left(\mathbf{k}_{1}, \mathbf{k}_{2}\right)\right| \leq \frac{C_{n}}{\left(\left|\mathbf{k}_{1}\right|\left|\mathbf{k}_{2}\right|\right)^{\operatorname{Re\nu }}}\left(\frac{1}{\left(1+\left|\mathbf{k}_{1}\right|+\left|\mathbf{k}_{2}\right|\right)^{n}}\right), \quad \text { for all } \mathbf{k}_{1} \mathbf{k}_{2} \in \mathbb{R}^{3} \backslash\{0\},
$$

finally, the constants $C_{n}$ depend continuously on $h$ with respect to the topology of compactly supported smooth functions on $M \times M$. This last observation can be proved using the continuity of the Fourier transform on $\Sigma_{\tau_{0}} \times \Sigma_{\tau_{0}}$ with respect to the Schwartz topology and the continuity of the causal propagator in the appropriate topologies, remembering that the restriction to $\Sigma_{\tau_{0}} \times \Sigma_{\tau_{0}}$ of the wavefunction $\Phi_{h}=$ $(E \otimes E) h$ is compactly supported.

We can now pass to consider the smooth restriction of $\Phi_{h}$ to $\Im^{-} \times \Im^{-}, \Psi_{h}$. Adapting a procedure similar to that we exploited for the wavefunctions, it arises that:

$$
\Psi_{h}\left(\ell, \omega, \ell^{\prime}, \omega^{\prime}\right)=H^{5} i \int_{\mathbb{R} \times \mathbb{R}} \frac{e^{-i \ell k_{1}-i \ell^{\prime} k_{2}}}{2 \pi} \sqrt{\frac{k_{1} k_{2}}{4}} \widetilde{\Phi}_{h}\left(H k_{1}, \eta(\omega), H k_{2}, \eta\left(\omega^{\prime}\right)\right) d k_{1} d k_{2} .
$$

The bound (75) entails some properties of $\widehat{\Psi}_{h}\left(k_{1}, \omega, k_{2}, \omega^{\prime}\right) \doteq i H^{5} \sqrt{\frac{k_{1} k_{2}}{4}} \widetilde{\Phi}_{h}\left(H k_{1}, \eta(\omega), H k_{2}, \eta\left(\omega^{\prime}\right)\right)$. The function $\widehat{\Psi}_{h}=\widehat{\Psi}_{h}\left(k_{1}, \omega, k_{2}, \omega^{\prime}\right)$ is everywhere smooth, except for $k_{1}=0$ or/and $k_{2}=0$, moreover the following angle-uniform bound holds, for every $n=1,2, \ldots$,

$$
\left|\widehat{\Psi}_{h}\left(k_{1}, \omega, k_{2}, \omega^{\prime}\right)\right| \leq \frac{C_{n}^{\prime}}{\left|k_{1} k_{2}\right|^{R e \nu-1 / 2}}\left(\frac{1}{\left(1+\left|k_{1}\right|+\left|k_{2}\right|\right)^{n}}\right),
$$

where each constant $C_{n}^{\prime}$ depends continuously on $h$ as before. Accordingly, it holds that $\widehat{\Psi}_{h}\left(k_{1}, \omega, k_{2}, \omega^{\prime}\right)$ is an integrable function on $\mathbb{R} \times \mathbb{S}^{2} \times \mathbb{R} \times \mathbb{S}^{2}$.

\section{Proofs of some propositions}

Proof of Lemma 3.1. As $E$ is the restriction to $M$ of the analogous causal propagator $\widehat{E}$ defined in the larger spacetime $\widehat{M}$ including $M$ as subspacetime, and since the singular support of $\widehat{E} \in \mathcal{D}^{\prime}(\widehat{M} \times \widehat{M})$ is given by the points $(y, x) \in \widehat{M} \times \widehat{M}$ such that there is a null $\widehat{g}$-geodesic connecting them, the lemma is proved if we establish that the null $\widehat{g}$-geodesic getting out from the compact $K \doteq \overline{\mathcal{O}} \subset M$ intersect $\Im^{-}$ in a compact set.

Let us prove this fact. To this end, as in [DMP08] and referring to the spacetime $\left(M, g_{F R W}\right)$ with coordinates and metric as in (2), we introduce the new null coordinates $U=\tan ^{-1}(\tau+r)$ and $V=$ $\tan ^{-1}(\tau-r)$ ranging in subsets of $\mathbb{R}$ individuated by $\tau \in(-\infty, 0)$ and $r \in(0,+\infty)$. Then:

$$
g_{F R W}=\frac{a^{2}(\tau(U, V))}{\cos ^{2} U \cos ^{2} V}\left[-\frac{1}{2} d U \otimes d V-\frac{1}{2} d V \otimes d U+\frac{\sin ^{2}(U-V)}{4} d \mathbb{S}^{2}(\theta, \varphi)\right] .
$$

$M \cup \Im^{-}$is the wedge $|V| \geq|U|$ with $V \in[-\pi / 2,0]$, but removing the boundary at $U=-V$ (including its endpoints) corresponding to $\tau=+\infty$, and omitting the point $(-\pi / 2,-\pi / 2)$, corresponding to the tip of $\Im^{-}$which does not exist in $\widehat{M}$. In this picture $\Im^{-}$coincides with the boundary at $V=-\pi / 2$ (without its endpoints), whereas the apparent boundary at $U=V$ is the submanifold $r=0$. However, with our choice of $a$, the metric in (77) is smooth outside this region, too. As a matter of fact, the globally hyperbolic spacetime $(\widehat{M}, \widehat{g})$, which extends $\left(M, g_{F R W}\right)$ is obtained letting $V$ ranging in a neighbourhood of $\Im^{-}$ 
including a region beyond it. The relevant point here is that also the metric $\widetilde{g}$ obtained cancelling the overall factor, $a^{2}(\tau(U, V)) /\left(\cos ^{2} U \cos ^{2} V\right)$, in the right-hand side of (77), is well-behaved and smooth for $U, V \in \mathbb{R}$ with $U \geq V$ (the apparent singularity for $U=V$ is a coordinate singularity only). The spacetime $(\widetilde{M}, \widetilde{g})$ obtained in that way is nothing but the (globally hyperbolic) Einstein static universe. The remarkable point is that, within this picture, $\Im^{-}$coincides with $\partial J^{+}\left(i^{-} ; \widetilde{M}\right) \backslash\left\{i^{-}\right\}=\partial I^{+}\left(i^{-} ; \widetilde{M}\right) \backslash\left\{i^{-}\right\}$, where the point $i^{-} \in \widetilde{M}$ is the tip of the cone $\Im^{-}$localised at $U=-\pi / 2, V=-\pi / 2$. Furthermore $M=I^{+}\left(i^{-} ; \widetilde{M}\right)$. An immediate consequence is that no future-directed null (or causal) $\widetilde{g}$-geodesics emanating from $K$ can reach $\Im^{-}$since $J^{+}(K ; \widetilde{M}) \subset I^{+}\left(i^{-} ; \widetilde{M}\right)$ which is always open and so, it cannot intersect $\partial I^{+}\left(i^{-} ; \widetilde{M}\right)=\Im^{-} \cup\left\{i^{-}\right\}$. Since the two metrics are conformally related [Wa84], the null geodesics of $(\widetilde{M}, \widetilde{g})$ when restricted to $\left(\widehat{M} \cup \Im^{-}, \widehat{g}\right)$ individuate $\widehat{g}$-geodesics and vice versa. Therefore our thesis would proved if we were able to establish that the past-directed null $\widehat{g}$-geodesic getting out from the compact $K \doteq \overline{\mathcal{O}} \subset M$ intersect $\Im^{-}$in a compact set (notice that $\Im^{-}$does not includes $i^{-}$).

Actually that result is true, since the analogous statement, for the opposite time orientation, was established in [Mo08] in the proof of Lemma 4.3 for a generic globally hyperbolic spacetime $\widetilde{M}$, when $M \subset \widetilde{M}$ is the globally hyperbolic subspacetime $I^{-}\left(i^{+} ; \widetilde{M}\right)$ with $i^{+} \in \widetilde{M}$ and $\Im^{+} \doteq \partial I^{-}\left(i^{+} ; \widetilde{M}\right) \backslash\left\{i^{+}\right\}=$ $\partial J^{-}\left(i^{+} ; \widetilde{M}\right) \backslash\left\{i^{+}\right\}$has the same geometric structure as $\Im^{-}$(referred to the opposite temporal orientation). In that case $K \subset M$ was any compact set (the further hypotheses assumed for $K$ in the proof of Lemma 4.3 in [Mo08] played no role in the part of the proof we are interested in).

Proof of Lemma 3.2. In the following, $\mathcal{E}(\ell, \omega, y)$ is the smooth integral kernel of $\Gamma E$ with the left entry restricted to $\Im^{-} \backslash \mathcal{N}_{\mathcal{O}_{h}}$ and the right one restricted to $\mathcal{O}_{h}$. To achieve (66), we start from:

$$
\begin{gathered}
\left|k_{1}\right|^{2}\left|k_{2}\right|^{2}|p|^{N}\left|(\Gamma E \otimes \Gamma E)\left(\left(\chi_{n}-\chi_{n_{h}}\right) e^{i\left\langle k_{1}, \cdot\right\rangle} \otimes\left(\chi_{n}-\chi_{n_{h}}\right) e^{i\left\langle k_{2}, \cdot\right\rangle} \otimes h e^{i\langle p, \cdot\rangle}\right)\right| \leq \\
\sum_{\alpha+\beta+\beta^{\prime}=N} \int_{M \times M} d \mu\left(y, y^{\prime}\right)\left|D_{y}^{\alpha} h(y)\right| \int_{\mathbb{S}^{2}} d \omega\left|\int_{\mathbb{R}} d \ell e^{i k_{1} \ell} \partial_{\ell}^{2} D_{y}^{\beta} \mathcal{E}\left(\ell, \omega, y_{1}\right)\left(\chi\left(\frac{\ell}{n}\right)-\chi_{n_{h}}(\ell)\right)\right| . \\
\cdot\left|\int_{\mathbb{R}} d \ell^{\prime} e^{i k_{2} \ell^{\prime}} \partial_{\ell^{\prime}}^{2} D_{y}^{\beta^{\prime}} \mathcal{E}\left(\ell^{\prime}, \omega, y_{2}\right)\left(\chi\left(\frac{\ell^{\prime}}{n}\right)-\chi_{n_{h}}\left(\ell^{\prime}\right)\right)\right| .
\end{gathered}
$$

where $y=\left(y_{1}, y_{2}\right) \in \mathcal{O}_{h} \times \mathcal{O}_{h}, d \mu\left(\ell, \ell^{\prime}, \omega, \omega^{\prime}, y, y^{\prime}\right) \doteq d \ell d S^{2}(\omega) d \ell^{\prime} d S^{2}\left(\omega^{\prime}\right) d \mu_{g}(y) d \mu_{g}\left(y^{\prime}\right)$ and, finally, $D_{y}$ are shortcut notations for the derivatives along the coordinates of $y$. To prove (66), since the domains of integration in $y, y^{\prime}$ and $\omega$ in the right-hand side of (78) have finite measure, it is enough proving that each of the two internal integrals in $d \ell$ and $d \ell^{\prime}$ give rise to functions of the remaining variables which are uniformly bounded in $n$. In other words we have now to establish that:

$$
\left|\int_{\mathbb{R}} d \ell e^{i k_{1} \ell} \partial_{\ell}^{2} D_{y}^{\beta} \mathcal{E}\left(\ell, \omega, y_{1}\right)\left(\chi\left(\frac{\ell}{n}\right)-\chi_{n_{h}}(\ell)\right)\right| \leq C_{\beta}<+\infty, \quad \text { uniformly in } n=1,2, \ldots
$$

To prove (79) we study the behaviour of the smooth function $\partial_{\ell}^{n} D_{y}^{\beta} \mathcal{E}(\ell, \omega, y)$. Starting form the analysis of the solutions of the Klein-Gordon equation in terms of modes summarised in Sec.2.1 and using results in the subsequent Sec.2.2, it arises that the causal propagator can be written, in the sense of the distributional $\epsilon$-prescription, i.e. smearing the kernel with a test function $g=g(y)$ before taking the limit, as

$$
D_{y}^{\beta} \partial_{\ell}^{m} \mathcal{E}(\ell, \omega, y)=\lim _{\epsilon \rightarrow 0^{+}} c \operatorname{Im}\left\{D_{y}^{\beta} \partial_{\ell}^{m} \int_{0}^{+\infty} d k e^{i 3 \pi / 4} e^{i k \ell / H} \sqrt{k} \chi_{k}(\tau) e^{-i k|\vec{y}| \cos (\omega, \vec{y})} e^{-\epsilon|k|}\right\},
$$

where $c \in \mathbb{R}$ is a constant irrelevant in our discussion, $y \doteq(\tau, \vec{y})$ and $\cos (\omega, \vec{y})$ is a shortcut notation for the cosine of the angle between $\vec{y}$ and the unit vector individuates by the angles $\omega \in \mathbb{S}^{2}$ when adopting 
spherical coordinate on the Cauchy surface $\Sigma_{\tau}$ the functions $\chi_{k}$ are the modes discussed in Sec.2.1. We have written $\mathcal{E}$ instead of $\Gamma E$, because we are intersted in the case $\left(\Im^{-} \backslash \mathcal{N}_{\mathcal{O}}\right) \times \mathcal{O}_{h} \ni((\ell, \omega), y)$ which implies that the kernel of $\Gamma E$ is smooth. We decompose the integral above into two parts $\int_{0}^{+\infty}=\int_{0}^{1}+\int_{1}^{+\infty}$ and we define

$$
D_{y}^{\beta} \partial_{\ell}^{m} \mathcal{E}_{<}(\ell, \omega, y) \doteq \lim _{\epsilon \rightarrow 0^{+}} c \operatorname{Im}\left\{D_{y}^{\beta} \partial_{\ell}^{m} \int_{0}^{1} d k e^{i 3 \pi / 4} e^{i k \ell / H} \sqrt{k} \chi_{k}(\tau) e^{-i k|\vec{y}| \cos (\omega, \vec{y})} e^{-\epsilon|k|}\right\},
$$

and

$$
D_{y}^{\beta} \partial_{\ell}^{m} \mathcal{E}_{>}(\ell, \omega, y) \doteq \lim _{\epsilon \rightarrow 0^{+}} c \operatorname{Im}\left\{D_{y}^{\beta} \partial_{\ell}^{m} \int_{1}^{+\infty} d k e^{i 3 \pi / 4} e^{i k \ell / H} \sqrt{k} \chi_{k}(\tau) e^{-i k|\vec{y}| \cos (\omega, \vec{y})} e^{-\epsilon|k|}\right\} .
$$

The case of $\varepsilon_{<}(\ell, \omega, y)$. The limit as $\epsilon \rightarrow 0^{+}$for first integral can be computed without using a smearing test function and the limit can be intechanged with the symbol of integral. This arises by direct application of Lebesgue's dominated convergence theorem. Furthermore, defining $a:=(\tau+\ell / H-$ $k|\vec{y}| \cos (\omega, \vec{y})$ ), we have the bound, following from (73) when $m=\beta=0$ :

$$
\begin{aligned}
& \lim _{\epsilon \rightarrow 0^{+}}\left|c \operatorname{Im} \int_{0}^{1} d k e^{i 3 \pi / 4} e^{i k \ell / H} \sqrt{k} \chi_{k}(\tau) e^{-i k|\vec{y}| \cos (\omega, \vec{y})} e^{-\epsilon|k|}\right|=\left|c \int_{0}^{1} d k e^{i 3 \pi / 4} e^{i k \ell / H} \sqrt{k} \chi_{k}(\tau) e^{-i k|\vec{y}| \cos (\omega, \vec{y})}\right| \\
& \leq|c| a^{\operatorname{Re\nu }-3 / 2} \frac{C_{\nu}}{2} \int_{0}^{a} u^{1 / 2-\operatorname{Re\nu }} d u+|c| a^{-3 / 2-\min (\operatorname{Re\nu }, 2)} \int_{0}^{a} O\left(k^{1 / 2+\min (\operatorname{Re\nu }, 2)}\right) d u,
\end{aligned}
$$

where, in our hypotheses $|a|>0$ since $|\ell|$ is very large wheras $y$ and $\omega$ range in a bounded domain (in other words $((\ell, \omega), y)$ does not belong to the singular support of $\Gamma E)$. The function in the second line of (81) vanishes as $|\ell| \rightarrow+\infty$ uniformly in $y \in \mathcal{O}_{h}$ and $\omega \in \mathbb{S}^{2}$. An analogous procedure can easily be implemented in presence ot derivatives $D_{y}^{\beta}, \partial_{\ell}^{m}$, making use of (73) again. The final result is that, for both $\nu \in i \mathbb{R}$ or $\nu \in(0,3 / 2)$, each function:

$$
\left(\Im^{-} \backslash \mathcal{N}_{\mathcal{O}}\right) \times \mathcal{O}_{h} \ni((\ell, \omega), y) \mapsto D_{y}^{\beta} \partial_{\ell}^{m} \mathcal{E}_{<}(\ell, \omega, y)
$$

vanishes as $|\ell| \rightarrow+\infty$ uniformly in $(\omega, y) \in \mathbb{S}^{2} \times \mathcal{O}_{h}$, so that it is bounded. Furthermore, if $m>0$, it is also $\ell$ integrable and the integral is bounded as a function of $(\omega, y) \in \mathbb{S}^{2} \times \mathcal{O}_{h}$.

Looking at the left-hand side of (79), we have the bound concerning the only contribution due to $D_{y}^{\beta} \mathcal{E}_{<}(\ell, \omega, y)$ :

$$
\begin{gathered}
\left|\int_{\mathbb{R}} d \ell e^{i k \ell} \partial_{\ell}^{2} D_{y}^{\beta} \mathcal{E}_{<}(\ell, \omega, y)\left(\chi\left(\frac{\ell}{n}\right)-\chi_{n_{h}}(\ell)\right)\right| \\
\leq \int_{\mathbb{R}} d \ell\left|\partial_{\ell}^{2} D_{y}^{\beta} \mathcal{E}_{<}(\ell, \omega, y)\right|\left|\chi\left(\frac{\ell}{n}\right)-\chi_{n_{h}}(\ell)\right|+2 \int_{\mathbb{R}} d \ell\left|\partial_{\ell} D_{y}^{\beta} \mathcal{E}_{<}(\ell, \omega, y)\right|\left|\partial_{\ell} \chi\left(\frac{\ell}{n}\right)-\partial_{\ell} \chi_{n_{h}}(\ell)\right|+ \\
+\int_{\mathbb{R}} d \ell\left|D_{y}^{\beta} \mathcal{E}_{<}(\ell, \omega, y)\right|\left|\partial_{\ell}^{2} \chi\left(\frac{\ell}{n}\right)-\partial_{\ell}^{2} \chi_{n_{h}}(\ell)\right|
\end{gathered}
$$

Let us start by analyzing the third integral in the right-hand side. Performing the change of variables $\ell \rightarrow n \ell$, it can be rewritten as:

$$
\int_{\mathbb{R}} d \ell\left|D_{y}^{\beta} \mathcal{E}_{<}\left(\ell, \omega, y_{1}\right)\right|\left|\partial_{\ell}^{2} \chi\left(\frac{\ell}{n}\right)-\partial_{\ell}^{2} \chi_{n_{h}}(\ell)\right|=\int_{\mathbb{R}} d \ell\left|D_{y}^{\beta} \mathcal{E}_{<}\left(n \ell, \omega, y_{1}\right)\right| n^{-1}\left|\partial_{\ell}^{2} \chi(\ell)-\partial_{\ell}^{2} \chi_{n_{h}}(n \ell)\right| .
$$


and the right-hand side is $n$-uniformly bounded by the product of $\sup _{(y, \omega) \in \mathcal{O}_{h} \times \Im^{-}}\left|D_{y}^{\beta} \mathcal{E}_{<}\right|-$which we know to be finite - and

$$
\frac{1}{n} \int_{\mathbb{R}} d \ell\left|\partial_{\ell}^{2} \chi(\ell)-\partial_{\ell}^{2} \chi_{n_{h}}(n \ell)\right| \leq \frac{1}{n} \int_{\mathbb{R}} d \ell\left|\partial_{\ell}^{2} \chi(\ell)\right|+\int_{\mathbb{R}} d s\left|\partial_{s}^{2} \chi_{n_{h}}(s)\right| \leq D<+\infty, \quad \forall n=1,2, \ldots
$$

The remaining two terms in the right hand side of (82) can be $n$-uniformly bounded similarly. The second term can be treated with an analogous procedure, with the change of variables $\ell \rightarrow n \ell$ using the fact that $\partial_{\ell} D_{y}^{\beta} \mathcal{E}_{<}(\ell, \omega, y)$ is bounded on $\left(\Im^{-} \backslash \mathcal{N}_{\mathcal{O}_{h}}\right) \times \mathcal{O}_{h}$ and that it holds $n^{-1} \int_{\mathbb{R}} d \ell\left|\partial_{\ell} \chi(\ell)-\partial_{\ell} \chi_{n_{h}}(n \ell)\right|<G<$ $+\infty$, uniformly in $n$. The first one can be treated analogously, noticing that $\left|\chi\left(\frac{\ell}{n}\right)-\chi_{n_{h}}(\ell)\right|$ is bounded uniformly $n$, whereas $\int_{\mathbb{R} \backslash\left[-\ell_{\mathcal{O}_{h}}, \ell_{\mathcal{O}_{h}}\right]} d \ell\left|\partial_{\ell}^{2} D_{y}^{\beta} \mathcal{E}_{<}(\ell, \omega, y)\right| \leq H<+\infty$ if $y \in \mathcal{O}_{h}$.

We have established that:

$$
\left|\int_{\mathbb{R}} d \ell e^{i k_{1} \ell} \partial_{\ell}^{2} D_{y}^{\beta} \mathcal{E}_{<}\left(\ell, \omega, y_{1}\right)\left(\chi\left(\frac{\ell}{n}\right)-\chi_{n_{h}}(\ell)\right)\right| \leq C<+\infty, \quad \text { uniformly in } n=1,2, \ldots
$$

To conclude it is enough to establish the analog for $\varepsilon_{>}$.

The case of $\varepsilon_{>}(\ell, \omega, y)$. We pass now to study the behaviour of $\varepsilon_{>}(\ell, \omega, y)$. As before we first examine the case $m=\beta=0$. To this end we exploit an approximation procedure to compute the modes $\chi_{k}(\tau)$ for $k>1$ which is similar to that used in [DMP08] and discussed in the Appendix A, but now using a different decomposition of the complete potential $k^{2}+a(\tau)^{2}\left[m^{2}+\left(\xi-\frac{1}{6}\right) R(\tau)\right]$ into ground and perturbation parts, $W_{0}(k)$ and $W(\tau)$, respectively. In fact, we define $W_{0}(k) \doteq k^{2}$ and $W(\tau) \doteq a(\tau)^{2}\left[m^{2}+\left(\xi-\frac{1}{6}\right) R(\tau)\right]$ in the differential equation in (9), so that the equation now reads:

$$
\frac{d^{2}}{d \tau^{2}} \chi_{k}(\tau)+\left(W_{0}(k)+W(\tau)\right) \chi_{k}(\tau)=0 .
$$

We stress that the modes $\chi_{k}$ are the same as that found employing the differential equation (9), only the perturbative procedure to solve it is different. The solution can be written as in (70) where now, $\chi_{k}^{0}(\tau) \doteq \frac{e^{-i \pi / 4}}{\sqrt{2 k}} e^{-i k \tau}, V$ is replced by $W$, and $S_{k}\left(t, t^{\prime}\right)$ is replaced by: $T_{k}\left(t, t^{\prime}\right) \doteq \frac{\sin \left(k\left(t-t^{\prime}\right)\right)}{2 k}$. By direct inspection one sees that the found series can be re-arranged as:

$$
e^{i \pi 4} \sqrt{k} \chi_{k}(\tau)=\sum_{n=0}^{\infty}\left(\frac{-1}{i k}\right)^{n}\left[A_{n}^{+}(k, \tau) e^{i k \tau}+A_{n}^{-}(k, \tau) e^{-i k \tau}\right],
$$

where the coefficient $A_{n}^{ \pm}$satisfy: $A_{0}^{+}(k, \tau) \doteq 0, A_{0}^{-}(k, \tau) \doteq 1$ and recursive relations:

$$
A_{n+1}^{+}(k, \tau)=\int_{-\infty}^{\tau} W(t)\left[A_{n}^{+}(k, t)+A_{n}^{-}(k, t) e^{-2 i k t}\right] d t, \quad A_{n+1}^{-}(k, \tau)=-\int_{-\infty}^{\tau} W(t)\left[A_{n}^{-}(k, t)+A_{n}^{+}(k, t) e^{2 i k t}\right] d t .
$$

All the integrand involved in the recursive procedure are absolutely integrable, due to the form of $W$ and the series (85) turns out to be uniformly absolutely convergent with its $t$-derivatives for $(t, k) \in$ $I\left(t_{0}\right) \times(1,+\infty)$, where $I\left(t_{0}\right)$ is a neighborhood of every $t_{0}<-T$, so that it can be derived under the symbol of series and, in this way, one can check that the left-hand side individuates a solution of (84). All the coefficient $A_{n}^{ \pm}(k, \tau)$ are bounded uniformly in $k$. To be more precise, the $k$ dependence of those coefficient appears as oscillating phases under some sign of integration. Furthermore there is always a part of $A_{n}^{-}(k, t)$ that does not depend on $k$ at all, while the coefficient $A_{n}^{+}(k, t)$ explicitly depends on $k$. We are now in place to discuss the behaviour of $\varepsilon_{>}(\ell, \omega, y)$ (always remaining out of the singularities of 
$\mathcal{E}(\ell, \omega, y)$, i.e. for $y \in \mathcal{O}_{h}$ and $\left.(\ell, \omega) \in \Im^{-} \backslash \mathcal{N}_{\mathcal{O}_{h}}\right)$. We have, for $y=(\tau, \vec{y})$,

$\partial_{\ell}^{m} D_{y}^{\beta} \mathcal{E}_{>}(\ell, \omega, y)=\lim _{\epsilon \rightarrow 0^{+}} c \operatorname{Im}\left\{\partial_{\ell}^{m} D_{y}^{\beta} \int_{1}^{\infty} d k i e^{i k \ell / H} \sum_{n=0}^{\infty}\left(\frac{-1}{i k}\right)^{n}\left[A_{n}^{+}(k, \tau) e^{i k \tau^{\prime}}+A_{n}^{-}(k, \tau) e^{-i k \tau^{\prime}}\right] e^{-\epsilon|k|}\right\}$,

where $\tau^{\prime} \doteq \tau-|\vec{y}| \cos (\omega, \vec{y})$. As before, we start by considering the case $m=0$ and $\beta=0$. The terms of the expansion of $\mathcal{E}_{>}$that could give rise to problems at large $k$ are only those with $n=0$ and $n=1$, the remaining rest $O\left(1 / k^{2}\right)$ produces $(y, \omega)$-uniformly bounded functions after evaluation of the integral (it can be evaluated without the $\epsilon$-prescription ). So, let us to examine:

$$
\lim _{\epsilon \rightarrow 0^{+}} c \operatorname{Im}\left\{\partial_{\ell}^{m} D_{y}^{\beta} \int_{1}^{\infty} d k i e^{i k \ell / H}\left[e^{-i k \tau^{\prime}}+\left(\frac{-1}{i k}\right)\left(A_{1}^{+}(k, \tau) e^{i k \tau^{\prime}}+A_{1}^{-}(k, \tau) e^{-i k \tau^{\prime}}\right)\right] e^{-\epsilon|k|}\right\},
$$

where $m=\beta=0$ and

$$
A_{1}^{+}(\tau, k):=\int_{-\infty}^{\tau} W(t) e^{-2 i k t} d t, \quad A_{1}^{-}(\tau, k):=-\int_{-\infty}^{\tau} W(t) d t
$$

and thus $A_{1}^{-}$does not depend on $k$, while $A_{1}^{+}$has an oscillating phase inside an integral. The first term in the integral (86) gives rise to a distribution proportional to:

$$
\delta(\ell / H-\tau+|\vec{y}| \cos (\omega, \vec{y}))-\frac{\sqrt{2}}{\sqrt{\pi}} \frac{\sin (\ell / H-\tau+|\vec{y}| \cos (\omega, \vec{y}))}{(\ell / H-\tau+|\vec{y}| \cos (\omega, \vec{y}))} .
$$

In this formula, actually, the Dirac delta cannot contribute because it is supported outside the domain we are considering, moreover the remaining term is bounded uniformly in $(y, \omega) \in \mathcal{O}_{h} \times \mathbb{S}^{2}$ and it falls off as $1 / \ell$ at large $\ell$. Let us now consider the term in the integral arising from $A_{1}^{-}$. It looks like

$$
A(\tau) \int_{1}^{\infty} \frac{\sin (k(\ell / H-\tau))}{k} d k
$$

which decays, for large $|\ell|$, faster then $1 /|\ell|^{\alpha}$, with $0 \leq \alpha<1$ so, it is $(y, \omega)$-uniformly bounded. The term containing $A_{1}^{+}$can also be easily shown to be $(y, \omega)$-bounded. That term can be written as

$$
\lim _{\epsilon \rightarrow 0^{+}} c \operatorname{Im}\left\{\int_{1}^{\infty} d k i e^{-\epsilon|k|} \int_{-\infty}^{\tau} e^{i k\left(\tau^{\prime}+\ell / H-2 t\right)} W(t)\right\} .
$$

Performing the $k$-integration, a distribution like (87) apperas, under the sign of integration in $t$ and it can also be shown to be bounded. Furthermore considering $m_{1} y$-derivatives and $m_{2} \ell$-derivatives of $\mathcal{E}_{>}$ does not affect its uniform boundedness, in particular, the extra terms of that series $n<m_{1}+m_{2}+2$ that needs to be taken into account, they can always treated as one of the case studied before, using the fact that the derivatives of the distribution (87) are either supported outside the support of $\mathcal{E}_{>}$or vanish uniformly for large $\ell$.

We conclude that, for $m=0,1$ and any $\beta$, every function:

$$
\left(\Im^{-} \backslash \mathcal{N}_{\mathcal{O}}\right) \times \mathcal{O}_{h} \ni((\ell, \omega), y) \mapsto D_{y}^{\beta} \partial_{\ell}^{m} \mathcal{E}_{>}(\ell, \omega, y)
$$

vanishes as $|\ell| \rightarrow+\infty$ uniformly in $(\omega, y) \in \mathbb{S}^{2} \times \mathcal{O}_{h}$, so that it is bounded. Looking at the left-hand side 
of (79), we have the bound concerning the only contribution due to $D_{y}^{\beta} \mathcal{E}_{>}(\ell, \omega, y)$ :

$$
\begin{gathered}
\left|\int_{\mathbb{R}} d \ell e^{i k \ell} \partial_{\ell}^{2} D_{y}^{\beta} \mathcal{E}_{>}(\ell, \omega, y)\left(\chi\left(\frac{\ell}{n}\right)-\chi_{n_{h}}(\ell)\right)\right| \leq \\
\leq\left|\int_{\mathbb{R}} d \ell e^{i k \ell}\left(\partial_{\ell}^{2} D_{y}^{\beta} \mathcal{E}_{>}(\ell, \omega, y)\right)\left(\chi\left(\frac{\ell}{n}\right)-\chi_{n_{h}}(\ell)\right)\right|+2 \int_{\mathbb{R}} d \ell\left|\partial_{\ell} D_{y}^{\beta} \mathcal{E}_{>}(\ell, \omega, y)\right|\left|\partial_{\ell} \chi\left(\frac{\ell}{n}\right)-\partial_{\ell} \chi_{n_{h}}(\ell)\right|+ \\
+\int_{\mathbb{R}} d \ell\left|D_{y}^{\beta} \mathcal{E}_{>}(\ell, \omega, y)\right|\left|\partial_{\ell}^{2} \chi\left(\frac{\ell}{n}\right)-\partial_{\ell}^{2} \chi_{n_{h}}(\ell)\right| .
\end{gathered}
$$

As before, we start by analyzing the third integral in the right-hand side. Performing the change of variables $\ell \rightarrow n \ell$, it can be rewritten as:

$$
\int_{\mathbb{R}} d \ell\left|D_{y}^{\beta} \mathcal{E}_{>}\left(\ell, \omega, y_{1}\right)\right|\left|\partial_{\ell}^{2} \chi\left(\frac{\ell}{n}\right)-\partial_{\ell}^{2} \chi_{n_{h}}(\ell)\right|=\int_{\mathbb{R}} d \ell\left|D_{y}^{\beta} \mathcal{E}_{<}\left(n \ell, \omega, y_{1}\right)\right| n^{-1}\left|\partial_{\ell}^{2} \chi(\ell)-\partial_{\ell}^{2} \chi_{n_{h}}(n \ell)\right|
$$

and the right-hand side is $n$-uniformly bounded by the product of $\sup _{(\omega, y) \in \mathbb{S}^{2} \times \mathcal{O}_{h}}\left|D_{y}^{\beta} \mathcal{E}_{>}\right|$- which we know to be finite - and

$$
\frac{1}{n} \int_{\mathbb{R}} d \ell\left|\partial_{\ell}^{2} \chi(\ell)-\partial_{\ell}^{2} \chi_{n_{h}}(n \ell)\right| \leq \frac{1}{n} \int_{\mathbb{R}} d \ell\left|\partial_{\ell}^{2} \chi(\ell)\right|+\int_{\mathbb{R}} d s\left|\partial_{s}^{2} \chi_{n_{h}}(s)\right| \leq D<+\infty, \quad \forall n=1,2, \ldots
$$

The second term in (88) can be treated with an analogous procedure, with the change of variables $\ell \rightarrow n \ell$ using the fact that $\partial_{\ell} D_{y}^{\beta} \mathcal{E}_{>}(\ell, \omega, y)$ is bounded on $\left(\Im^{-} \backslash \mathcal{N}_{\mathcal{O}_{h}}\right) \times \mathcal{O}_{h}$ and that it also holds $n^{-1} \int_{\mathbb{R}} d \ell\left|\partial_{\ell} \chi(\ell)-\partial_{\ell} \chi_{n_{h}}(n \ell)\right|<G<+\infty$, uniformly in $n$.

To conclude, in order to establish that:

$$
\left|\int_{\mathbb{R}} d \ell e^{i k \ell} \partial_{\ell}^{2} D_{y}^{\beta} \mathcal{E}_{>}(\ell, \omega, y)\left(\chi\left(\frac{\ell}{n}\right)-\chi_{n_{h}}(\ell)\right)\right| \leq C<+\infty, \quad \text { uniformly in } n=1,2, \ldots
$$

so concluding the overall proof, it is sufficient to prove a $n$-uniform bound for the first term in the right-hand side of (88):

$$
\left|\int_{\mathbb{R}} e^{i k \ell}\left(\partial_{\ell}^{2} D_{y}^{\beta} \varepsilon_{>}(\ell, \omega, y)\right)\left(\chi\left(\frac{\ell}{n}\right)-\chi_{n_{h}}\right) d \ell\right| \leq C(\beta) \text {, uniformly in } n \text { and in } k .
$$

Without loosing generality we shall substitute the $y$ derivative with $i k$ factors in the $k$-expansion of $\mathcal{E}_{>}$. The terms not considered here are harmless, in particular, the case when the $y$ derivative is a $\tau$ derivative that acts on the first of the recursive integrals in the perturbative series can only lower the degree of divergence. We use the expansion found beforehand with $w=\beta+2$ :

$$
\begin{gathered}
\lim _{\epsilon \rightarrow 0^{+}} \mid \int_{\mathbb{R}} e^{i k \ell} \operatorname{Im}\left\{\int_{1}^{\infty} d k i e^{i k \ell / H}(i k)^{w} \sum_{m=0}^{\infty}\left(\frac{-1}{i k}\right)^{n}\left[A_{m}^{+}\left(k, \tau^{\prime}\right) e^{i k \tau^{\prime}}+A_{m}^{-}\left(k, \tau^{\prime}\right) e^{-i k \tau^{\prime}}\right] e^{-\epsilon|k|}\right\} . \\
\cdot\left(\chi\left(\frac{\ell}{n}\right)-\chi_{n_{h}}\right) d \ell \mid
\end{gathered}
$$

The coefficient $A_{m}^{-}\left(k, \tau^{\prime}\right)$ decompose into the sum $A_{m}^{-}(k, \tau)=B_{m}\left(\tau^{\prime}\right)+B_{m}^{-}\left(k, \tau^{\prime}\right)$, where $B_{m}\left(\tau^{\prime}\right)$ does not depend on $k$. We start considering only these $k$-independent terms.

$$
\begin{gathered}
\lim _{\epsilon \rightarrow 0^{+}} \mid \int_{\mathbb{R}} e^{i k \ell}\left\{\int_{\mathbb{R}} d k \Theta(|k|-1)(i k)^{w} e^{i k \ell / H} \sum_{m=0}^{\infty}\left(\frac{-1}{i k}\right)^{n} B_{m}(\tau) e^{-i k \tau^{\prime}} e^{-\epsilon|k|}\right\} . \\
\cdot\left(\chi\left(\frac{\ell}{n}\right)-\chi_{n_{h}}\right) d \ell \mid
\end{gathered}
$$


Notice that the preceding integral can be seen as a the convolution between $\widehat{\mathcal{E}}$ and $\widehat{\chi}_{n}-\widehat{\chi}_{n_{h}}$,

$$
\lim _{\epsilon \rightarrow 0^{+}}\left|\int_{\mathbb{R}} \Theta(|q|-1) \sum_{m=0}^{\infty}(-1)^{m}(i q)^{w-m} B_{m}(\tau) e^{-i q \tau} e^{-\epsilon|q|}\left(n \widehat{\chi}(n(k-q))-\widehat{\chi}_{n_{h}}(k-q)\right) d q\right| .
$$

We now divide the sum above as $\sum_{w-m \geq 0}+\sum_{w-m<0}$ and analyze the second sum.

$$
\begin{gathered}
\lim _{\epsilon \rightarrow 0^{+}}\left|\int_{\mathbb{R}} \Theta(|q|-1) \sum_{m=w+1}^{\infty}(-1)^{m}(i q)^{w-m} B_{m}(\tau) e^{-i q \tau} e^{-\epsilon|q|}\left(n \widehat{\chi}(n(k-q))-\widehat{\chi}_{n_{h}}(k-q)\right) d q\right| \leq \\
\leq \sum_{m=w+1}^{\infty}\left|B_{m}\left(\tau^{\prime}\right)\right| \int_{\mathbb{R}}|n \widehat{\chi}(n(k-q))|+\left|\widehat{\chi}_{n_{h}}(k-q)\right| d q=C(w)
\end{gathered}
$$

where, in the last term, we have used the fact that the series of the continuous functions $\tau^{\prime} \mapsto\left|B_{n}\left(\tau^{\prime}\right)\right|$ converges uniformly so that the sum is continuous and thus it admits a finite bound when $(\omega, y) \in \mathbb{S}^{2} \times \mathcal{O}_{h}$ (remind that $\left.\tau^{\prime}=\tau-|\vec{y} \cos (\omega, y)|\right)$, and that the last integral does not depend both on $q$ and $n$. The first term in the series can be treated analogously exploiting the fact that $q^{m} \Theta(|q|-1)=q^{m}-q^{m} \Theta(1-|q|)$ and noticing that $q^{m}$ is the Fourier transform of the $m$ th-derivative of the delta distribution supported outside the support of $\chi_{n}-\chi_{n_{h}}$, hence it cannot give any contribution. Finally, it remains to consider:

$$
\begin{gathered}
\lim _{\epsilon \rightarrow 0^{+}}\left|\int_{\mathbb{R}} \Theta(1-|q|) \sum_{m=0}^{w}(-1)^{m}(i q)^{w-m} B_{m}\left(\tau^{\prime}\right) e^{-i q \tau} e^{-\epsilon|q|}\left(n \widehat{\chi}(n(k-q))-\widehat{\chi}_{n_{h}}(k-q)\right) d q\right| \leq \\
\leq \sum_{m=0}^{w}\left|B_{m}\left(\tau^{\prime}\right)\right| \int_{\mathbb{R}}|n \widehat{\chi}(n(k-q))|+\left|\widehat{\chi}_{n_{h}}(k-q)\right| d q=C^{\prime}(w)
\end{gathered}
$$

The remaining term present in the perturbative series, those associated with $B_{n}^{-}(q, \tau)$ and $A_{n}^{+}(q, \tau)$, can be treated analogously, apart for the contribution of the delta functions. It is in fact not always true that the support of the derivatives of the deltas stay outside the support of the $\chi \mathrm{s}$, but in this case, the delta distributions appear inside one of the recursive integrals, similarly to the case of the proof of boundedness of $A_{1}^{+}(k, t)$ considered before, hence they are harmless.

\section{References}

[Al85] B. Allen, "Vacuum states in the Sitter space", Phys. Rew. D 32 (1985) 3136.

[BST83] J. M. Bardeen, P. J. Steinhardt and M. S. Turner, "Spontaneous Creation Of Almost Scale Free Density Perturbations In An Inflationary Universe," Phys. Rev. D 28, (1983) 679.

[BGP96] C. Bär, N. Ginoux, F. Pfäffle, "Wave equations on Lorentzian manifolds and quantization", ESI Lectures in Mathematics and Physics, European Mathematical Society Publishing House (2007).

[BS06] A.N. Bernal, M. Sánchez, "Further Results on the Smoothability of Cauchy Hypersurfaces and Cauchy Time Functions", Lett. Math. Phys. 77, (2006) 183.

[BR021] O. Bratteli, D. W. Robinson, "Operator Algebras And Quantum Statistical Mechanics. Vol. 1: $C^{*}$ And $W^{*}$ Algebras, Symmetry Groups, Decomposition Of States", second edition, Springer-Verlag (2002). 
[BR022] O. Bratteli, D. W. Robinson, "Operator algebras and quantum statistical mechanics. Vol. 2: Equilibrium states. Models in quantum statistical mechanics", second edition, Springer (2002).

[BF00] R. Brunetti and K. Fredenhagen, "Microlocal analysis and interacting quantum field theories: Renormalization on physical backgrounds," Commun. Math. Phys. 208, (2000) 623.

[BFK96] R. Brunetti, K. Fredenhagen and M. Köhler, "The microlocal spectrum condition and Wick polynomials of free fields on curved spacetimes," Commun. Math. Phys. 180 (1996) 633.

[BFV03] R. Brunetti, K. Fredenhagen, R. Verch. "The Generally covariant locality principle: A New paradigm for local quantum field theory." Commun. Math. Phys. 237 (2003) 31.

[BD78] T.S. Bunch and P.C.W. Davies, "Quantum Fields theory in de Sitter space: renoramlization by point-splitting", Proc. R. Soc. Lond. A 360 (1978) 117.

[DFP08] C. Dappiaggi, K. Fredenhagen and N. Pinamonti, "Stable cosmological models driven by a free quantum scalar field," Phys. Rev. D 77 (2008) 104015.

[DMP06] C. Dappiaggi, V. Moretti and N. Pinamonti, "Rigorous steps towards holography in asymptotically flat spacetimes", Rev. Math. Phys. 18 (2006) 349.

[DMP08] C. Dappiaggi, V. Moretti and N. Pinamonti, "Cosmological horizons and reconstruction of quantum field theories", arXiv:0712.1770 [gr-qc], to appear on Comm. Math. Phys.

[Da07] C. Dappiaggi, "Projecting massive scalar fields to null infinity", Ann. Henri. Poinc. 9 (2008) 35.

[DR03] M. Duetsch and K. H. Rehren, "Generalized free fields and the AdS-CFT correspondence", Annales Henri Poincare 4 (2003) 613.

[GR95] I. S. Gradshteyn, I. M. Ryzhik, Table of Integrals, Series, and Products, Fifth Edition, Academic Press, (1995).

[Ho00] S. Hollands: "Aspects of Quantum Field Theory in Curved Spacetime", Ph.D. Thesis - University of York (2000), advisor B.S. Kay, unpublished.

[HW05] S. Hollands, R.M. Wald: "Conservation of the stress tensor in interacting quantum field theory in curved spacetimes". Rev. Math. Phys. 17, (2005) 227.

[HW02] S. Hollands and R. M. Wald, “An alternative to inflation,” Gen. Rel. Grav. 34 (2002) 2043.

[HW01] S. Hollands and R. M. Wald, "Local Wick polynomials and time ordered products of quantum fields in curved spacetime," Commun. Math. Phys. 223 (2001) 289.

[Ho93] G. 't Hooft, "Dimensional reduction in quantum gravity," arXiv:gr-qc/9310026.

[Hö71] L. Hörmander, “Fourier integral operators. I.", Acta Math. 127, (1971) 79.

[Hö89] L. Hörmander, "The Analysis of Linear Partial Differential Operators", Vol I, (1989) Springer.

[JS01] W. Junker and E. Schrohe, "Adiabatic vacuum states on general spacetime manifolds: Definition, construction, and physical properties," Annales Poincare Phys. Theor. 3 (2002) 1113.

[KW91] B. S. Kay, R. M. Wald, "Theorems On The Uniqueness And Thermal Properties Of Stationary, Nonsingular, Quasifree States On Space-Times With A Bifurcate Killing Horizon", Phys. Rept. 207 (1991) 49. 
[KiGa93] K. Kirsten and J. Garriga, "Massless minimally coupled fields in de Sitter space: O(4) symmetric states versus de Sitter invariant vacuum," Phys. Rev. D 48 (1993) 567.

[Kü08] M. Küskü, "A class of almost equilibrium states in Robertson-Walker spacetimes," Desy-Thesis2008-020

[Li96] A. Linde: "Particle Physics and Inflationary Cosmology", Harwood Academic Publishers (1996).

[LR90] C. Lüders and J. E. Roberts, "Local quasiequivalence and adiabatic vacuum states," Commun. Math. Phys. 134, (1990) 29.

[Mo03] V. Moretti, "Comments on the stress energy tensor operator in curved space-time". Commun. Math. Phys. 232, (2003) 189.

[Mo06] V. Moretti, "Uniqueness theorem for BMS-invariant states of scalar QFT on the null boundary of asymptotically flat spacetimes and bulk-boundary observable", Commun. Math. Phys. 268, (2006) 726 .

[Mo08] V. Moretti, "Quantum out-states states holographically induced by asymptotic flatness: Invariance under spacetime symmetries, energy positivity and Hadamard property", Commun. Math. Phys. $27 \mathbf{9}$ (2008) 31.

[MP03] V. Moretti and N. Pinamonti, "Quantum Virasoro algebra with central charge $c=1$ on the event horizon of a 2D Rindler spacetime," J. Math. Phys. 45, (2004) 257.

[MFB92] V. F. Mukhanov, H. A. Feldman and R. H. Brandenberger, "Theory of cosmological perturbations. Part 1. Classical perturbations. Part 2. Quantum theory of perturbations. Part 3. Extensions," Phys. Rept. 215, (1992) 203.

[NO83] A. Nikiforov and V. Ouvarov, "Fonctions spéciales de la physique mathé matique" Éditions Mir, Moskou, (1983).

[Ol07] H. Olbermann, "States of Low Energy on Robertson-Walker Spacetimes," Class. Quant. Grav. 24, (2007) 5011.

[Rad96a] M. J. Radzikowski, "Micro-Local Approach To The Hadamard Condition In Quantum Field Theory On Curved Space-Time," Commun. Math. Phys. 179 (1996) 529.

[Rad96b] M. J. Radzikowski, "A Local to global singularity theorem for quantum field theory on curved space-time," Commun. Math. Phys. 180 (1996) 1.

[Reh00] K. H. Rehren, "Local quantum observables in the anti-deSitter - conformal QFT correspondence," Phys. Lett. B 493 (2000) 383.

[Ri07] P. L. Ribeiro, "Structural and Dynamical Aspects of the AdS/CFT Correspondence: a Rigorous Approach," arXiv:0712.0401 [math-ph] Ph.D Thesis - University of Sao Paolo.

[Ri06] W. Rindler, "Relativity. Special, General and Cosmological", Second Edition, Oxford University Press, (2006).

[Ru73] W. Rudin, "Functional Analysis", McGraw-Hill Book, New York (1973).

[SV01] H. Sahlmann, R.Verch "Microlocal spectrum condition and hadamard form for vector valued quantum fields in curved space-time", Rev. Math. Phys. 13, (2001) 1203. 
[SA08] J.A. Sanders "Aspects of locally covariant quantum field theory", Ph.D Thesis - University of York (2008), advisor C.J. Fewster.

[SS76] C. Schomblond and P. Spindel "Conditions d'unicité pour le propagateur $\Delta^{(1)}(x, y)$ du champ scalaire dans l'univers de de Sitter", Ann. Inst. Henri Poincaré 25, (1976) 67.

[SVW02] A.Strohmaier, R.Verch, M. Wollenberg "Microlocal analysis of quantum fields on curved spacetimes: analytic wavefront sets and Reeh-Schlieder theorems", J.Math. Phys. 43 (2002) 5514.

[Wa84] R. M. Wald: "General Relativity”, Chicago University Press (1984).

[Wa94] R. M. Wald: "Quantum field theory in curved space-time and black hole thermodynamics", The University of Chicago Press (1994). 